\title{
3 Perfekt \& Präteritum im Alemannischen Deutschlands
}

In diesem Kapitel untersuche ich die jüngere (sprachgeschichtliche) Entwicklung und die Semantik des Präteritums im Alemannischen Deutschlands. Zunächst wird die Entwicklung des Präteritums von 1888 bis 2013 beschrieben (Kap. 3.1). Dazu werden Ortsgrammatiken und Untersuchungen der Jahre 1888 bis 1982 ausgewertet und die Ergebnisse aus meinen Untersuchungskorpora angeschlossen. Ich betrachte dabei die areale Ausbreitung und die Frequenz der Präteritumformen im Vergleich mit den Perfektformen. Zusätzlich wird die Frage behandelt, welche Gemeinsamkeiten die im Präteritum vorkommenden Verben haben. Im Anschluss daran untersuche ich die Semantik der Tempora Perfekt und Präteritum. Dabei sollen lediglich die Verben berücksichtigt werden, bei denen Präteritumformen zu finden sind (Kap. 3.2). Ein Fazit hält die wesentlichen Ergebnisse dieses Kapitels fest (Kap. 3.3). Da ich in diesem Kapitel 3 die Tempora Perfekt und Präteritum betrachte, erfasse ich die Präteritalformen der Hilfsverben sein und haben zur Bildung des Plusquamperfekts nicht. In Kapitel 4.2.4 wird beim Vergleich der Hilfsverben, die zur Bildung der Tempora Plusquamperfekt und Doppelperfekt gebraucht werden, näher auf die Präteritalformen der Hilfsverben sein und haben eingegangen. Die Präteritalformen der Hilfsverben sein und werden zur Passivbildung werden dagegen in diesem Kapitel mit behandelt, da bei diesen - im Gegensatz zu den Präteritalformen zur Plusquamperfektbildung - Variation zwischen Präteritum und Perfekt möglich ist und das Tempus an diesen Hilfsverben markiert wird. Die folgenden Beispielsätze (20) verdeutlichen das:

(20) a. Er wurde besiegt. vs. Er ist besiegt worden.

b. Sie war besiegt. vs. Sie ist besiegt gewesen.

$\mathrm{Da}$, wie gerade verdeutlicht, die Tempusmarkierung bei Passivkonstruktionen an den Hilfsverben sein und werden markiert wird, behandle ich diese Hilfsverben gemeinsam mit den Kopulaverben sein und werden. Auf die Tempusvariation beim Passiv werde ich in Kapitel 3.2.6 näher eingehen. 


\subsection{Jüngere Entwicklung (1974-2013)}

\subsubsection{Forschungsstand}

Jüngere Untersuchungen des Präteritums im Alemannischen Deutschlands fehlen m. W. vollständig. Vor allen Dingen Georg Wenkers Sprachatlas des Deutschen Reichs (1888-1923) (vgl. Fischer 2018: 15) und die Ortsgrammatiken des alemannischen Sprachraums (vgl. Rowley 1983: 164-165 und Fischer 2018: 37-39) haben zur nun vorherrschenden Meinung beigetragen, im Alemannischen würden (genau wie im gesamten oberdeutschen Sprachraum) lediglich die Präteritalformen von sein vereinzelt noch verwendet werden (vgl. Rowley 1983: 165 und Fischer 2018: 38-39).

Die folgende Karte 5 gibt einen Überblick über die von Fischer ausgewerteten Ortsgrammatiken. In den meisten Untersuchungsgebieten war kein Präteritum festzustellen. Einige Ortsgrammatiken dokumentieren ein Präteritum von sein. Dieses ist zum Teil als neue Form markiert (Fischer 2018: 37). Mehne stellt beispielsweise für die Grundmundart keine Präteritumformen fest. Allerdings trete das Präteritum von sein häufiger in standardnäheren Sprachschichten auf (Mehne 1954: 55).

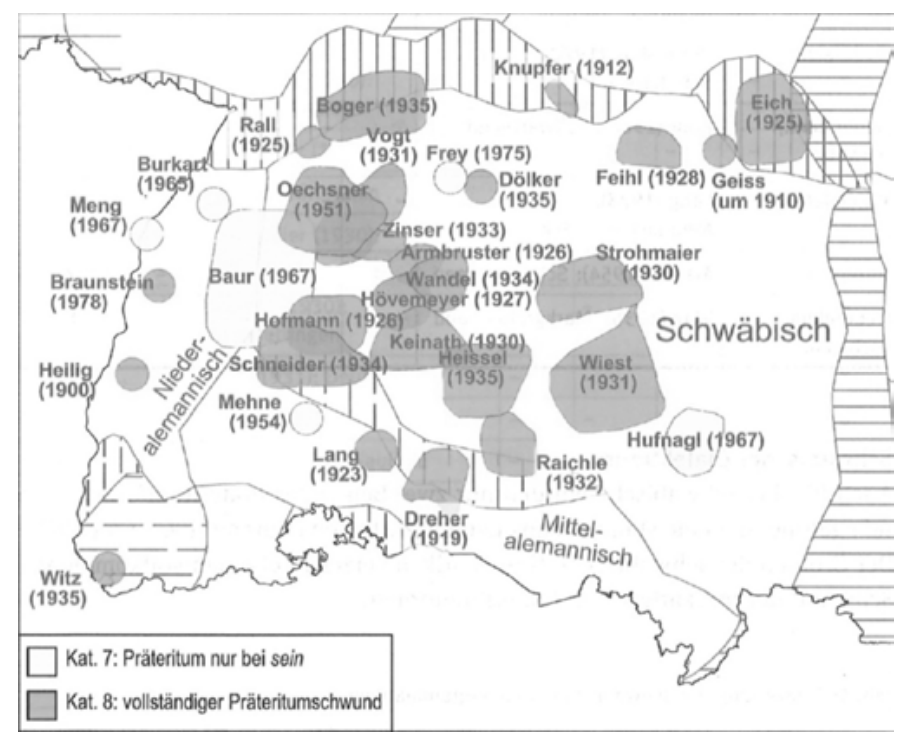

Karte 5: Präteritumschwund nach Ausweis der alemannischen und schwäbischen Dialektgrammatiken (Fischer 2018: 37) 
Eine Gegenposition zur weitverbreiteten Annahme, das Präteritum sei im Alemannischen ausgestorben, nimmt Gersbachs Untersuchung (1982) ein. Er wertet das Zwirner Korpus mit Tonaufnahmen freier Gespräche aus dem Alemannischen Deutschlands, dem Fränkischen und dem Alemannischen im Vorarlberg aus (Deutsches Spracharchiv; Erhebungszeitraum 1955-1964). Dabei kommt er zu dem Schluss, dass von einem absoluten Fehlen des Präteritums im Oberdeutschen nicht (mehr) die Rede sein könne (Gersbach 1982: 224). Vielmehr beschränke sich das Präteritum auf ein paar wenige Verben. Gersbach ermittelt insgesamt 52 Verben; davon sind jedoch 34 nur einmal belegt. Das Verb sein weist 92,6\% aller Präteritumformen (4224 Belege) auf. Die übrigen 51 Lemmata machen dagegen nur 7,4\% der Präteritalformen aus (338 Belege). Die häufigsten dieser Lemmata sind die Verben kommen (68), gehen (43), werden (39), müssen (38) und wollen (30) (ebd.: 84-85). Gersbach vergleicht die Frequenz der Präteritalformen mit denen der Perfektformen nur beim Verb sein in einem Teilkorpus. Dort entfallen 1226 Belege auf das Präteritum (52,8\%) und 1095 auf das Perfekt (47,2\%) (Gersbach 1982: 94). Im Alemannischen Deutschlands, im Bairisch-Schwäbischen und im Schwäbischen ist die Präteritumfrequenz besonders hoch (ebd.: 91). Das Vorhandensein des Präteritums begründet Gersbach durch einen Einfluss aus dem Standard, da mit „steigender Sprachschicht [...] Prät. in der Gebrauchshäufigkeit zunimmt“ (ebd.: 217).

Der Vergleich der Ergebnisse von Gersbach (1982) und der oben aufgeführten Mundartgrammatiken zeigt also Unterschiede im Gebrauch des Präteritums für das Alemannische Deutschlands. Wie sind diese zu erklären?

Denkbar wäre, dass das Präteritum in der Zeit zwischen der Erstellung der Ortsgrammatiken, in denen kein Präteritum festgestellt wurde, und Gersbachs Untersuchung wieder in Gebrauch gekommen ist. $\mathrm{Zu}$ dieser These passt die Tatsache, dass in der Mehrzahl der jüngeren Ortsgrammatiken (1951-1978) ein Präteritum von sein festgestellt wurde.

Tab. 3: Präteritum in den Mundartgrammatiken des Alemannischen Deutschlands von 1951$1978^{11}$

\begin{tabular}{llll}
\hline Dialektraum & Autor/Jahr & Region/Ort & Präteritum von sein \\
\hline Mittelalemannisch & Mehne (1954) & Schwenningen & $\begin{array}{l}\text { nicht vorhanden/im } \\
\text { Aufbau/neu } \\
\text { vorhanden }\end{array}$ \\
\hline
\end{tabular}

11 Diese Tabelle ist angelehnt an die Auswertung Fischers (2018: 38-39). 


\begin{tabular}{llll}
\hline Dialektraum & Autor/Jahr & Region/Ort & Präteritum von sein \\
\hline $\begin{array}{l}\text { Niederalemannisch } \\
\text { Niederalemannisch }\end{array}$ & Meng (1967) & Auenheim & im Aufbau/neu \\
$\begin{array}{l}\text { Niederalemannisch/ } \\
\text { Schwäbisch }\end{array}$ & Baur (1967) & Schwarzwald & im Aufbau/neu \\
Schwäbisch & Oechsner (1951) & Nagold-Enzgebiet & nicht vorhanden \\
Schwäbisch & Hufnagl (1967) & Memmingen & vorhanden \\
Schwäbisch & Frey (1975) & Stuttgart & vorhanden \\
Schwäbisch & Vogt (1977) & Schwäbisch & nicht vorhanden \\
\hline
\end{tabular}

Dass die Mundartgrammatiken im Gegensatz zu Gersbach (1982) keine Präteritalformen anderer Verben nachweisen, ließe sich durch deren geringere Datenmenge erklären. Obwohl Gersbach nur beim Verb sein die Frequenz der Präteritalformen mit denen der Perfektformen vergleicht, ist anzunehmen, dass der Anteil an Präteritalformen im Vergleich zu den Perfektformen der anderen Verben extrem gering ist. Schließt man die Belege für sein aus, finden sich in Gersbachs Korpus 338 Präteritalformen und 35207 Perfektformen (vgl. Gersbach 1982: 224). Das Verhältnis von Präteritum zu Perfekt ist bei Gersbach also $1: 104,16$. Angesichts der geringen Frequenz der Präteritalformen ist bei deutlich kleinerer Datenmenge durchaus denkbar, dass bei der Erhebung der Ortsgrammatiken kein Präteritum beobachtet werden konnte.

Gegen die These, dass das Präteritum in der Zeit zwischen der Erstellung der Ortsgrammatiken, in denen kein Präteritum festgestellt wurde, und Gersbachs Untersuchung wieder gebräuchlich wurde, spricht allerdings, dass in Vogts Grammatik des Schwäbischen das Präteritum als „absolut nicht gebräuchlich“ (Vogt 1977: 122) bezeichnet wird, obwohl Gersbach mit Aufnahmen aus den Jahren 1955 bis 1964 ältere Daten auswertet. Zudem darf zumindest angezweifelt werden, ob zwischen den älteren Ortsgrammatiken, in denen überhaupt kein Präteritum auffindbar war, und Gersbachs Untersuchung (1982) ein Präteritum von sein wieder so gebräuchlich wurde, dass es häufiger verwendet wird als dessen Perfektform. 
Tab. 4: Präteritum in den Mundartgrammatiken des Alemannischen Deutschlands von 1930$1947^{12}$

\begin{tabular}{llll}
\hline Dialektraum & Autor/Jahr & Region/Ort & Präteritum von sein \\
\hline Bodenseealemannisch & $\begin{array}{l}\text { Gruber ([1942- } \\
\text { 47)] 1989) }\end{array}$ & Westallgäu & nicht vorhanden \\
Mittelalemannisch & Witz(1935) & Markgräflerland & nicht vorhanden \\
Mittelalemannisch/ & Schneider (1934) & Liggersdorf & nicht vorhanden \\
Schwäbisch & & & \\
Schwäbisch & Strohmaier (1930) & Blaubeuren & nicht vorhanden \\
Schwäbisch & Keinath (1930) & Onstmettingen & nicht vorhanden \\
Schwäbisch & Vogt (1931) & Deufringen & nicht vorhanden \\
Schwäbisch & Wiest (1931) & Burgrieden & nicht vorhanden \\
Schwäbisch & Raichle (1932) & Saulgau & nicht vorhanden \\
Schwäbisch & Zinser (1933) & Ammertal & nicht vorhanden \\
Schwäbisch & Wandel (1934) & Reutlingen-Betzingen & nicht vorhanden \\
Schwäbisch & Dölker (1935) & Esslingen a. Necker & nicht vorhanden \\
Schwäbisch & Heissel (1935) & Friedingen & nicht vorhanden \\
Schwäbisch/Rhein-fränkisch & Boger (1935) & Enz-Pfinz-Gebiet & nicht vorhanden \\
\hline
\end{tabular}

Neben der eben beschriebenen Möglichkeit eines Sprachwandels kann das fehlende Präteritum in den meisten Ortsgrammatiken auch mit methodischen Unterschieden zwischen Gersbachs Untersuchung (1982) und den Mundartgrammatiken begründet werden. Gersbach stellt bei einem Vergleich einiger Ortsgrammatiken und Untersuchungen für dieselben Gebiete teilweise große Unterschiede in der Beschreibung des Gebrauchs beziehungsweise Schwunds des Präteritums fest (Gersbach 1982: 43-49). Er führt diese Unterschiede auf unterschiedliche Explorationsmethoden zurück, die in den Mundartgrammatiken häufig nicht explizit gemacht würden (ebd.: 44). Da sich viele dieser Untersuchungen vor allen Dingen auf das Lautsystem des jeweiligen Ortsdialekts konzentrieren, wurden Daten durch direkte Wort- oder Satzabfragen erhoben (vgl. Strohmaier 1930, Boger 1935 und Hufnagl 1967). Bei dieser Art der Datenerhebung lässt sich Tempusvariation kaum ermitteln. Gersbach vermutet, dass bei vielen Erhebungen vielleicht nicht gezielt nach Präteritumformen gefragt wurde, weil die Exploratoren ohnehin von einem vollständigen Präteritumschwund ausgegangen

12 Diese Tabelle ist angelehnt an die Auswertung Fischers (2018: 38-39). 
seien (Gersbach 1982: 49). Tatsächlich basieren viele Ortsgrammatiken allerdings nicht auf Befragungen von Gewährspersonen, sondern auf der Introspektion der Verfasser (Schmidt/Herrgen 2011: 90-97 und Fischer 2018: 27). Fischer weist auf folgende Unklarheiten bei vielen Mundartgrammatiken hin: Erstens sei nicht klar, welchen Status die angegebenen Formen haben, da selten systematisch zwischen Erinnerungs-, Kompetenz- und Performanzformen unterschieden werde. Auch vertikale oder generationelle Variation werde nicht systematisch ausgewiesen. Zweitens sei unklar, ob Verbformen vollständig vorhanden sind. Zum Teil könne nicht entschieden werden, ob nicht-aufgeführte Formen im Dialekt nicht vorhanden seien oder ob der Verfasser sie nur nicht dokumentiert habe (Fischer 2018: 28). Es ist also fraglich, ob Präteritalformen erst Mitte des 20. Jahrhunderts im Alemannischen wieder gebräuchlich oder ob sie lediglich nicht erfasst wurden.

Neben Sprachwandel und methodischen Unterschieden in der Erhebung könnten die Präteritumformen bei Gersbach (1982) auch durch standardnahe Sprechweisen der Gewährspersonen erklärt werden. Er teilt diese in drei Sprachschichten ein und weist die Präteritumfrequenz getrennt für die drei Sprachschichten aus. Ein Vergleich der Ortsgrammatiken mit Gersbachs Untersuchung ist nur bei der standardfernsten Sprachschicht von Gersbach möglich. Doch auch bei dieser ermittelt er Präteritumformen. Allerdings macht Gersbach keine Angaben darüber, ob diese Präteritumformen in standardnahen Sprechweisen geäußert wurden. Dass auch oberdeutsche Sprecher das Präteritum kennen, macht unter anderem Widén deutlich. Er verweist darauf, dass oberdeutsche Sprecher aufgrund ihrer Schulbildung das Präteritum bilden können und dieses nutzen, um vornehm zu klingen (Widén 1970: 283-284). In den Aufnahmesituationen von Gersbachs (1982) Untersuchung ist also durchaus denkbar, dass einige der Gewährspersonen - unabhängig von ihrer Sprachschicht - in standardnahe Sprechweisen übergehen und dort Präteritalformen verwenden. Da Gersbach nicht angibt, ob die von ihm beobachteten Präteritalformen aus ländlichen Gegenden kommen, in denen traditionell standardferner gesprochen wird, oder aus größeren Städten (vgl. Gersbach 1982: 216), stellt Rowley die Vermutung an, es handle sich bei den von Gersbach gefundenen Präteritumformen um Belege aus dem Stadtdialekt (Rowley 1983: 165).

Die Frage, warum in den älteren Ortsgrammatiken kein Präteritum genannt wird, muss zunächst unbeantwortet bleiben. Allerdings lässt sich zweifelsfrei feststellen, dass es spätestens seit den 1950er Jahren ein Präteritum für wenige Verben gibt (vgl. Gersbach 1982). Unklar ist, ob diese Präteritalformen in natürlicher Sprache oder in affektierter standardnaher Sprechweise produziert wurden. 
Die mir zur Verfügung stehenden Daten lassen sich gut mit denen aus Gersbachs (1982) Untersuchung vergleichen, da sich die Erhebungsmethoden sehr ähneln (vgl. Kap. 1.2). Ich ziehe die beiden Korpora SSA und REDI+FLARS (dt.) für die Analyse heran. Die Korpora FR und REDI+FLARS (frz.) werden für die Untersuchung des Präteritums ausgeschlossen, da das Korpus FR auch standardnahe Sprecher aus Freiburg enthält und im Elsass, abgesehen von einigen wenigen Präteritalformen von haben und sein, keine Präteritalformen zu finden sind.

Ich werde zunächst überprüfen, ob die Präteritalformen aus meinen Korpora als Standardmarker dienen können. Dies wäre der Fall, wenn Präteritumformen vermehrt in Äußerungen zu finden sind, die auch aufgrund anderer sprachlicher Merkmale als standardnah zu bewerten sind. Diese Verwendung von Präteritumformen in standardnaher Sprechweise werden für die weitere Untersuchung ausgeschlossen, da sie als Code-Switching beziehungsweise Code-Shifting vom Dialekt zum Standard zu bewerten ist (vgl. Schwitalla 2012: 48) (Kap. 3.1.2).

Danach untersuche ich die areale Ausbreitung des Präteritums und behandle die Frage, ob die Anzahl der Ortspunkte, in denen Präteritum zu finden ist, gegenüber den Ortspunkten, in denen kein Präteritum zu finden ist, zugenommen hat (Kap. 3.1.3). Daraufhin soll der von Rowley (1983) gestellten Frage nachgegangen werden, ob die Präteritumformen nur in Städten vorzufinden sind. Ich vergleiche dabei die Einwohnerzahl der Orte mit der Anzahl an Verben, die in diesen verwendet wird (Kap. 3.1.4).

Gersbach vermutet mit Verweis auf Ruoff (1975: 253) ein Vordringen des Präteritums seit Ende des 1. Weltkriegs (Gersbach 1982: 216). Sollte diese Vermutung zutreffen, müsste sowohl die Frequenz der Präteritumformen als auch der Formenbestand diachron zunehmen. Wie oben bereits beschrieben, stellt Gersbach abgesehen vom Verb sein keine Frequenzvergleiche zwischen den Präteritumund Perfektformen eines Verbs an. Daher können seine Ergebnisse nur bedingt mit meinen verglichen werden. Allerdings bietet sich ein Vergleich meiner Untersuchungskorpora an. Ich werde daher überprüfen, ob die Tokenfrequenz der Präteritumformen im jüngeren Korpus REDI+FLARS (dt.) (mit Aufnahmen von 20072013) gegenüber der des älteren Korpus SSA (mit Aufnahmen von 1974-1985) zugenommen hat und ob die Anzahl der Verben, mit denen Präteritum gebildet wird, gestiegen ist (Kap. 3.1.5). Darüber hinaus gehe ich der Frage nach, welche Gemeinsamkeiten die präteritumaffinen Verben im gegenwartssprachlichen Badischalemannischen haben (Kap. 3.1.6).

Im Anschluss daran wird untersucht, ob eine höhere Präteritumfrequenz mit einem Rückgang der Dialektalität korreliert, d. h. ob standardnähere Sprecher tendenziell häufiger Präteritum gebrauchen als standardfernere Sprecher. Als 
Marker für Dialektalität ziehe ich die Partizipien der Perfektformen heran und ermittle, ob es sich um Standard- oder Dialektformen handelt (Kap. 3.1.7).

Um die Frage zu beantworten, ob die Präteritalformen aus dem Standard entlehnt wurden oder nie völlig aus dem Dialekt verschwunden sind und seit einiger Zeit an Häufigkeit zunehmen, untersuche ich, ob die Präteritum-Belege Standard- oder Dialektformen sind. Bei einer Entlehnung der Formen aus dem Standard wäre zu erwarten, dass sie keine oder nur eine sehr geringe Assimilation an das Alemannische erfahren haben. Auf der anderen Seite sprächen Dialektformen des Präteritums (z. B. der Indikativ Präteritum von sein als was, wie er im Schwäbischen Wörterbuch (Fischer 1920: 1330) und in der Mundartgrammatik von Memmingen (Hufnagl 1967: 132) als alte und erhaltene Form beschrieben wird) dafür, dass das Präteritum im Alemannischen nie völlig verschwunden ist, sondern nur nicht erfasst wurde (Kap. 3.1.8). Abschließend fasse ich die Ergebnisse dieses Kapitels zusammen (Kap. 3.1.9).

\subsubsection{Das Präteritum in der standardnahen Sprechweise von Dialektsprechern}

Wie oben dargelegt wurde, herrscht die Meinung vor, es gebe im Alemannischen höchstens ein Präteritum des Verbs sein. Im „waschechten“ (Vogt 1977: 122) Dialekt sei das Präteritum nicht gebräuchlich. Allerdings würden oberdeutsche Sprecher Präteritum verwenden, um formell und schriftdeutsch/standarddeutsch zu klingen (Widén 1970: 283 und Fischer 2018: 213). Der Wille, sich vornehm auszudrücken, führe häufig auch zu hyperkorrekten Verwendungen des Präteritums, d. h. Verwendungen mit Gegenwartsbezug (vgl. Schmeller 1821: 375, Trier 1965: 205, Hentschel/Weydt 2013: 95-96 und Fischer 2018: 213-214). Auch in der Laienlinguistik finden sich zahlreiche Verweise auf das Fehlen des Präteritums im Dialekt. Dementsprechend wird es als ,nicht zum Dialekt gehörig' und ,hochgestochen' bezeichnet. ${ }^{13}$ Es stellt sich daher zuerst die Frage, ob die Präteritalformen, die in meinen Untersuchungskorpora zu finden sind, Teil des eigentlichen Dialekts sind oder ob diese als Code-Switching beziehungsweise Code-Shifting vom Dialekt zum Standard zu bewerten sind.

13 Vgl. hierzu die folgenden Ausschnitte der Frage-Antwort-Plattformen, auf denen Laien Fragen stellen und beantworten können: https://german.stackexchange.com/questions/977/wann-wird-pr\%C3\%A4teritum-in-bayern-verwendet und http://www.uni-protokolle. de/foren/viewt/159527,0.html sowie einen Artikel aus Miss Moneypenny. Die Schweizer Fachzeitschrift für Office Managerinnen und Assistentinnen (http://www.missmoneypenny.ch/article/praeteritum-und-drumherum). 
Um diese Frage zu beantworten, werde ich in diesem Kapitel Präteritalformen in standardnahen von Präteritalformen in dialektalen Sprechweisen abgrenzen. Wie in Kapitel 1.2 beschrieben wurde, sind alle Gewährspersonen kompetente Dialektsprecher. Sie wechseln jedoch vereinzelt von ihrer dialektnahen in eine standardnahe Sprechweise. Diese standardnahen Sprechweisen reichen von kurzen Äußerungen bis zu kleineren oder größeren Gesprächsphasen. Ich habe nur die Äußerungen als standardnah klassifiziert, die neben möglicherweise standardnahen Präteritalformen noch weitere standardsprachliche Merkmale aufweisen. Standardnahe Präteritalformen, die in dialektaler Sprechweise geäußert werden, betrachte ich als Entlehnungen aus dem Standard.

Schwitalla unterscheidet zwischen plötzlichem (=Code-Switching) und stufenweisem (=Code-Shifting) Wechsel der Varietät (vgl. Schwitalla 2012: 48). Allerdings ist fraglich, ob diese Unterscheidung immer gemacht werden kann. Dabei gilt: Je ähnlicher zwei Varietäten sind, desto schwieriger ist es, einen genauen Zeitpunkt zu ermitteln, an dem ein Wechsel von Varietät A zu Varietät B geschieht. Beim Wechsel vom Dialekt zum Standard und umgekehrt ist die Ermittlung eines Wechselpunktes dementsprechend in vielen Fällen unmöglich, da sich die Strukturen ähneln und viele Begriffe in beiden Varietäten homophon sind. So kann oft nicht entschieden werden, welche Varietät zu einem bestimmten Punkt gesprochen wird (Auer 2010: 461-462). Eine Unterscheidung in CodeSwitching und Code-Shifting ist daher häufig nicht möglich. Da ich in diesem Kapitel nur Präteritalformen in (fast) standardsprachlichen von dialektalen Sprechweisen abgrenzen möchte, ist eine exakte Unterscheidung zwischen Code-Switching und Code-Shifting allerdings auch nicht notwendig. Aus diesem Grund schließe ich alle Präteritalformen aus, die in (fast) standardsprachlicher Sprechweise geäußert werden, unabhängig davon, ob ein abrupter oder ein kontinuierlicher Wechsel vom Dialekt zu dieser standardsprachlichen Äußerung erfolgt ist.

Nicht jeder Wechsel der Varietät muss eine kommunikative Bedeutung haben (vgl. Bürkli 1999: 288 und Schwitalla 2012: 49, FN 17). Dennoch möchte ich in diesem Kapitel einen Einblick geben, warum Sprecher vom Dialekt zum Standard wechseln. Die folgenden Beispiele sind dementsprechend nicht als umfassende Darstellung aller Funktionen zu betrachten. Zuerst stelle ich Fälle vor, in denen Präteritalformen in der natürlichen dialektalen und anschließend in (fast) standardsprachlicher Sprechweise geäußert werden.

\section{Präteritalformen in dialektalen Äußerungen}

In Beispiel (21) berichtet der Sprecher GP1_97 von seinen Aufzeichnungen über strukturelle Veränderungen durch die Post, Telefon und die Eisenbahnerfindung (Z. 03). 
(21) Altheim bei Riedlingen (SSA)

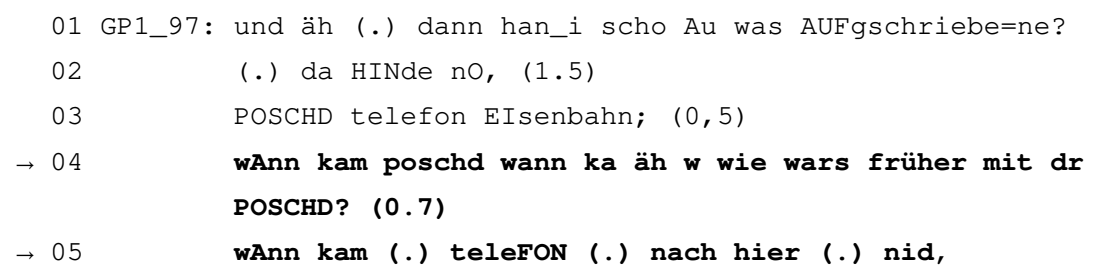

GP1_97 erzählt, er habe aufgeschrieben (Z. 01), wann die Post (Z. 04) und wann das Telefon kamen (Z. 05). In den Äußerungen in den Zeilen 04 und 05 verwendet er die Präteritalform „kam“. Hierbei handelt es sich nicht um einen Wechsel in den Standard. Dialektale Merkmale dieser Äußerungen sind die Palatalisierung in „poschd“, die Realisierung des auslautenden [r] und der Tilgung des [e] in „dr“ (Z. 04) sowie die question tag „nid“ (Z. 05). Diese Merkmale finden sich auch in anderen Äußerungen (vgl. „POSCHD telefon EIsenbahn“ in Zeile 03).

Auch im nächsten Beispiel (22) verwendet die Sprecherin eine Präteritalform in dialektaler Sprechweise (Z. 14). Hier erzählt sie von Verständigungsschwierigkeiten, die sie bei einem Besuch in Köln hatte.

(22) Auenheim (REDI+FLARS (dt.))

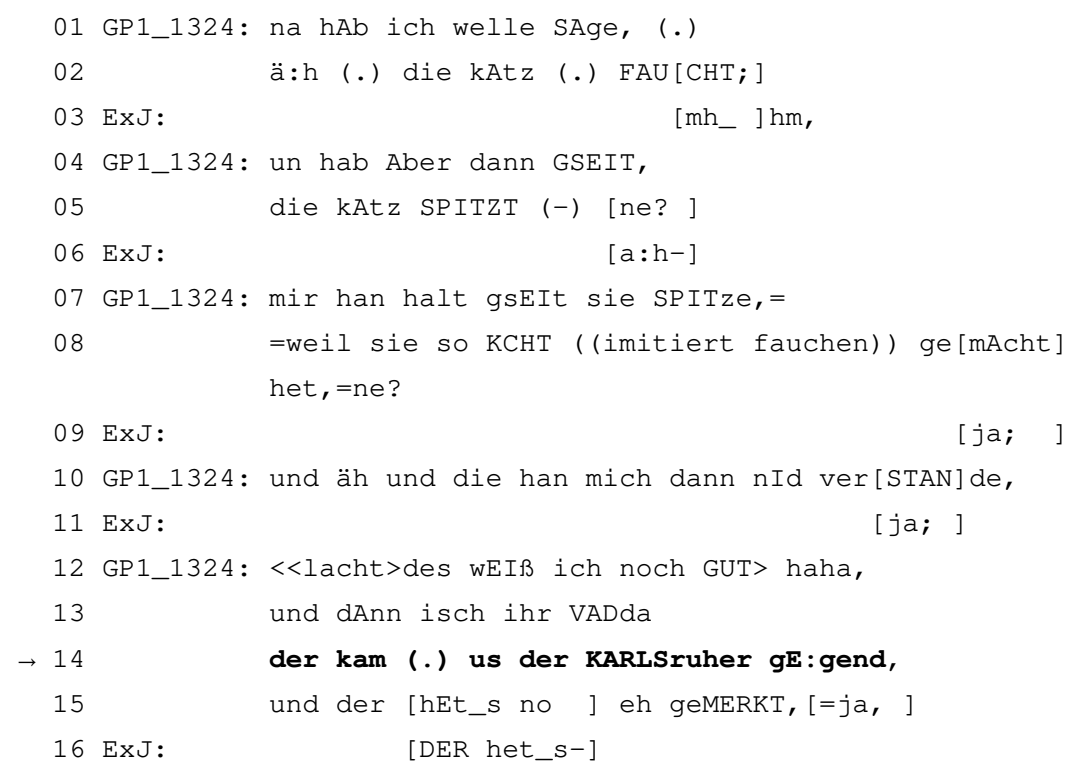


18 GP1_1324: no wAs_ich MEIN;

GP1_1324 erzählt, sie wäre einmal zu Besuch in Köln gewesen und habe anstelle der standarddeutschen Variante „FAUCHT“ (Z. 02) die dialektale „SPITZT“ (Z. 05) verwendet. Lediglich der Vater einer Freundin hätte sie damals verstanden, da er aus der Karlsruher Gegend kam (Z. 13-17). Der Großteil der Äußerung in Zeile 14 ist in der dialektalen Sprechweise homophon zur standardsprachlichen. Abgesehen von der Präteritalform „kam“ liefert nur die Monophthongierung von aus zu „us“ (Z. 14) einen Hinweis darauf, dass es sich hierbei um eine dialektale Äußerung handelt. Daher ist auch diese Äußerung nicht als Wechsel der Varietät zu betrachten.

\section{Präteritalformen in (fast) standardsprachlichen Äußerungen}

Ein abrupter Wechsel von einer Varietät zu einer anderen geschieht häufig beim Zitieren anderer Personen (vgl. Schwitalla 2012: 48). Das folgende Beispiel (23) illustriert einen solchen plötzlichen Wechsel. Die beiden Gewährspersonen erzählen im Vorfeld dieses Ausschnitts von einer Bekannten, die ihre Kinder zuhause keinen Dialekt sprechen lässt, da sie glaubt, in der Schule würden sie andernfalls ,schlechtes Deutsch' sprechen. Diese Ansicht wird von der Interviewerin EX1_1051 und dem Sprecher GP1_1162 nicht geteilt. GP2_1162 leitet daraufhin in Zeile 01 einen Widerspruch ein und berichtet von einem Bekannten, der tatsächlich Probleme mit dem Standarddeutschen in der Schule hatte (Z. 0308). In diesem Transkript habe ich phonetische Zeichen integriert, da sie für die Analyse von Bedeutung sind.

(23) Herrischried (REDI+FLARS (dt.))

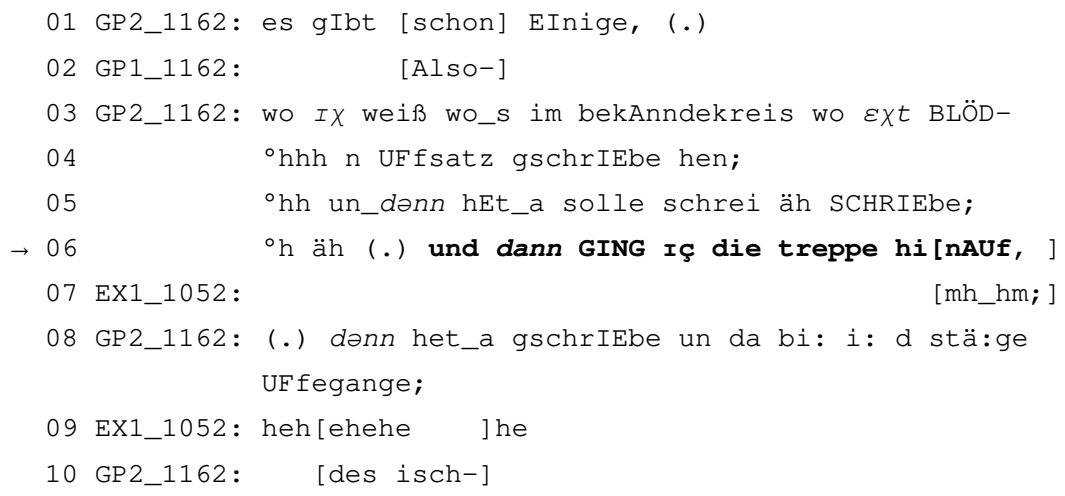


GP2_1162 erzählt, einer ihrer Bekannten hätte bei Aufsätzen in der Schule anstelle des zu erwartenden standarddeutschen Satzes „und dann GING Iç die treppe hinAUf“ (Z. 06) die dialektale Variante „un da bi: i: d stä:ge UFfegange“ (Z. 08) geschrieben. Dabei wechselt die Gewährsperson zweimal abrupt die Varietät: zum einen in den Standard, um den erwartbaren standarddeutschen Satz zu zitieren, und zum anderen in einen standardferneren Dialekt als ihren eigenen, um ihren Bekannten zu zitieren. Das standardsprachliche Zitat ist durch die Verzögerungspartikel „äh“ und die Mikropausen vor und nach dem Zitat von ihrer üblichen Sprachlage getrennt. Während sie hier (Z. 01 und 03-05) bei den Wörtern „I $\chi “$

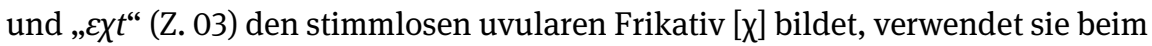
Zitieren des Wortes „IÇ“ (Z. 06) den standardsprachlichen stimmlosen palatalen Frikativ [ç]. Auch beim standardsprachlichen „dann“ (Z. 06) zeigen sich Unterschiede zu ihrer üblichen Sprache, in der sie dieses Wort mit Schwa [ə] (Z. 05 und 08) anstelle des Vollvokals [a] ausspricht. Zudem ist „hinAUf“ eine standardsprachliche Bildung. An früherer Stelle und im Zitat ihres Bekannten äußert GP2_1162 stattdessen die Verbpartikel „UFfe“.

Auch bei Reformulierungen finden sich Wechsel vom Dialekt zum Standard (vgl. Schwitalla 2012: 49-50). In Beispiel (24) erzählt der Sprecher GP1_86 von seinem Heimatort Vöhrenbach.

(24) Vöhrenbach (SSA)

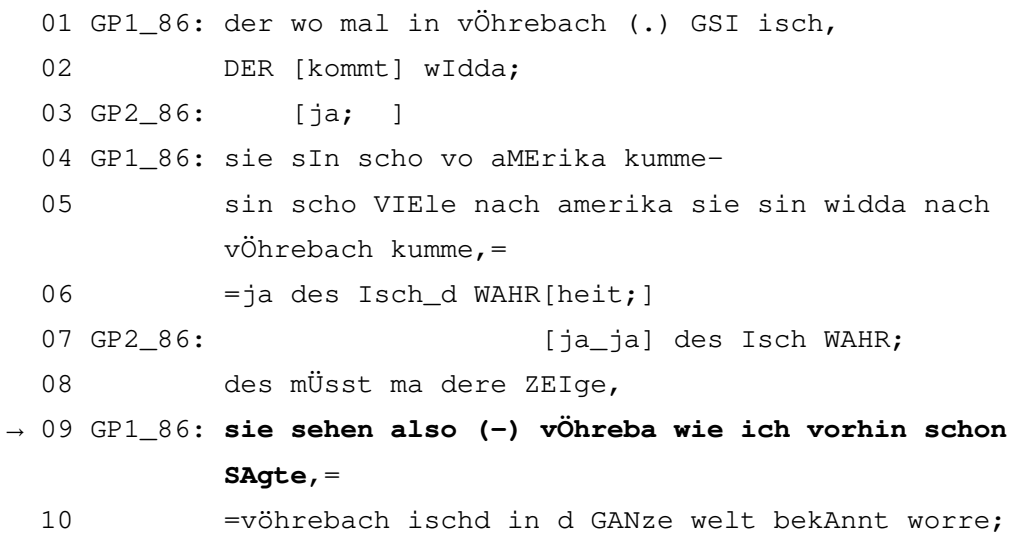

Im bisherigen Gespräch erwähnte GP1_86 bereits mehrfach, dass Vöhrenbach sehr bekannt sei. In den Zeilen 01-02 erzählt GP1_86 in seiner üblichen standardfernen Sprache, dass alle, die einmal in Vöhrenbach gewesen seien, wieder dorthin zurückkämen. Er führt diese Behauptung in den Zeilen 04-06 weiter aus und berichtet davon, dass viele nach Amerika gegangen und wieder nach 
Vöhrenbach zurückgekehrt seien. Daraufhin leitet er in Zeile 09 eine Zusammenfassung seiner vorherigen Aussagen ein, Vöhrenbach sei weltweit bekannt. Bei dieser Äußerung in Zeile 09 handelt es sich um einen metakommunikativen Verweis auf vorangegangene Teile des Gesprächs (vgl. Kap. 4.3.5). Während er seine restliche Erzählung dialektal äußert, wechselt er für den diskursiven Verweis in den Standard. Das ist zum einen daran zu erkennen, dass „schon“ (Z. 09) anders als in den Zeilen 04 und 05 sowie „sehen“ (Z. 09) nicht reduziert wurden. Zum anderen handelt es sich bei „vorhin“ (Z. 09) um eine standardsprachliche Variante des dialektalen Ausdrucks ebbe, den GP1_86 an anderer Stelle des Gesprächs benutzt. Die Zusammenfassung (,vöhrebach ischd in d GANze welt bekAnnt worre") äußert er wieder in seiner üblichen Sprache.

Ein Wechsel vom Dialekt zum Standard kann auch einen Sequenzabschluss markieren (Kallmeyer/Keim 1994: 162 und 164 sowie Schwitalla 2012: 92). Das ist in folgendem Beispiel (25) zu sehen. Hier erzählt die Sprecherin, dass ihr Vater früher eine Versicherungsagentur hatte und sie sonntags mit ihm das Geld von den Versicherten einsammeln musste. Der Gesprächsausschnitt beginnt mit der Erzählung eines dieser Besuche.

(25) Elzach (REDI+FLARS (dt.))

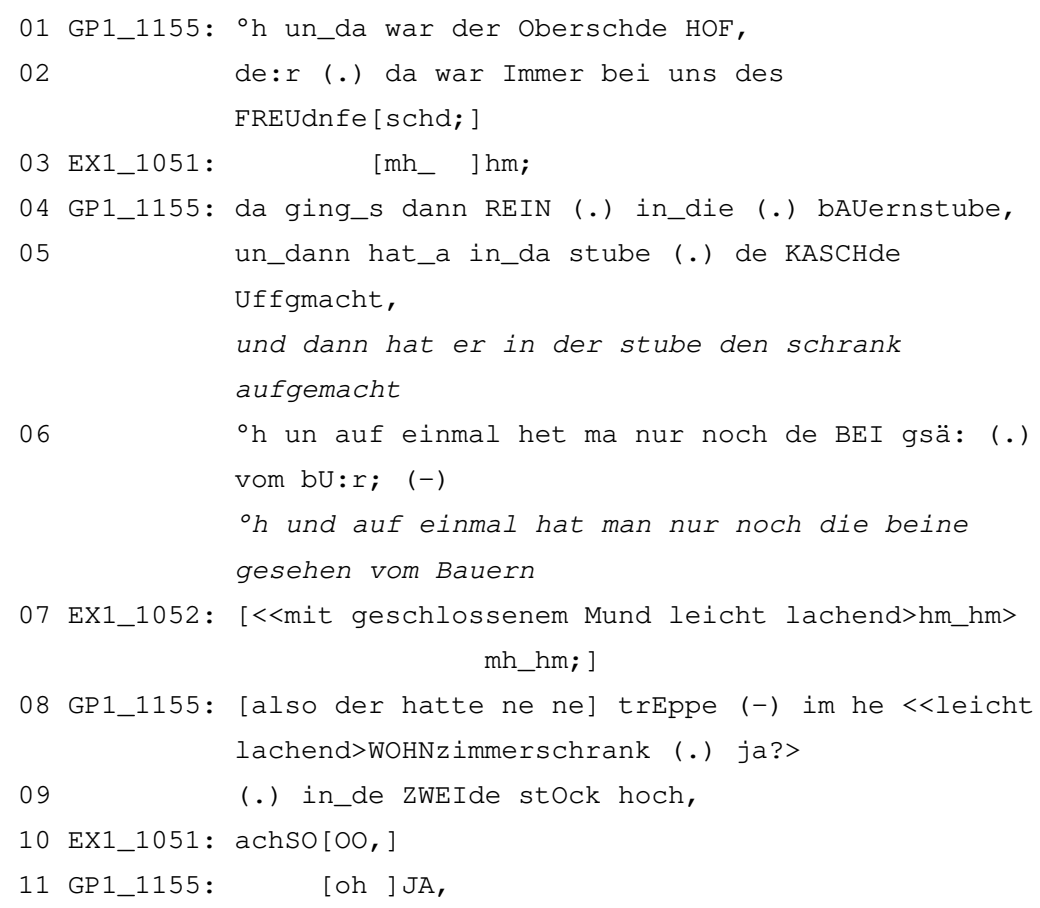




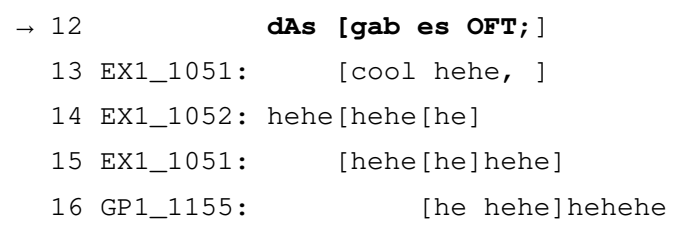

GP1_1155 erzählt, wie sie mit ihrem Vater zu einem Gasthof gekommen sei (Z. 0104). Dort habe der Besitzer eine Schranktür geöffnet (Z. 05) und sei hineingeklettert (Z. 06). Die Äußerung in Zeile 06 bildet die Pointe ihrer Episode. Das ist an der fallenden Intonation und der darauffolgenden kurzen Pause, in der GP1_1155 den Turn abgeben will, zu sehen. Die Interviewer scheinen diese Pointe allerdings nicht (als solche) zu verstehen, da EX1_1052 nur mit einem verhaltenen Lachen und einem Rezeptionssignal reagiert (Z. 07) und EX1_1051 überhaupt nicht. Aus diesem Grund erklärt GP1_1155 die Pointe, indem sie erzählt, es habe sich um eine Treppe im Wohnzimmerschrank gehandelt (Z. 08-09). Nach dieser Erklärung signalisiert EX1_1051 durch ein langgezogenes „achSOOO“ (Z. 10) Verstehen. GP1_1155 schließt ihre Episode daraufhin in standardsprachlicher Sprechweise ab (Z. 12). Ihre Äußerung in Zeile 12 und das darauffolgende gemeinsame Lachen (Z. 14-16) bilden den Abschluss dieser Sequenz.

Im Gegensatz zu ihrer restlichen Erzählung ist ihre abschließende Äußerung standardsprachlich. Die Standardnähe wird durch den standardsprachlichen definiten Artikel „dAs“ (Z. 12) deutlich. Zudem weist sie, im Gegensatz zum Großteil ihrer restlichen Erzählung, keine wortübergreifenden Verschleifungen auf. So ist das Pronomen „es“ (Z. 12) anders als in Zeile 04 nicht klitisiert.

Wir haben gesehen, dass Sprecher aus verschiedenen Gründen von ihrer üblichen dialektalen Sprechweise in den Standard oder in eine standardnahe Sprechweise wechseln. Das kann unter anderem zum Zitieren standardnaher Sprecher, zur Initiierung einer Reformulierung oder zum Abschließen einer Sequenz geschehen. Die Präteritalformen, die in diesen standardnahen Sprechweisen verwendet werden, können nicht als Teil des Alemannischen betrachtet werden. Daher schließe ich solche standardnahen Äußerungen aus der folgenden Untersuchung aus. Im Folgenden werde ich die Präterital- und Perfektformen untersuchen, die in dialektaler Sprechweise geäußert werden.

\subsubsection{Areale Ausbreitung}

In Kapitel 3.1.1 wurden die Fragen aufgeworfen, in welchen Gebieten das Präteritum zu finden ist und ob das Präteritum seit Mitte des 20. Jahrhunderts wieder 
ins Alemannische Deutschlands eingedrungen ist. Neben einem diachronen Vergleich scheint insbesondere der Unterschied zwischen ländlichen und städtischen Gebieten interessant zu sein, da in Städten traditionell standardnäher gesprochen wird als in ländlichen Gegenden. Es muss deshalb die Frage beantwortet werden, ob sich diese Reinnovation auf Städte begrenzt.

Ich präsentiere in diesem Kapitel zunächst die areale Ausbreitung einiger Verben, ehe ich in Kapitel 3.1.4 auf den möglichen Unterschied zwischen kleineren und größeren Orten eingehe. Ich vergleiche jeweils die Verteilung im SSA und in REDI+FLARS (dt.). Da hinsichtlich der arealen Ausbreitung und einem möglichen Unterschied zwischen kleineren und größeren Orten keine Unterschiede zwischen der älteren und der jüngeren Sprechergruppe in REDI+FLARS (dt.) zu erwarten sind, behandle ich sie in diesem Kapitel gemeinsam.

Fischer (2018) stellt für den Abbau des Präteritums eine Hierarchie zwischen verschiedenen Verbgruppen fest. Diese lassen sich nach ihrer Resistenz gegenüber dem Präteritumschwund in folgender Reihenfolge anordnen: das Kopulaverb sein, Modalverben, das Hilfsverb haben, irreguläre Verben, starke Verben und schwache Verben (Fischer 2018: 381). An dieses Ergebnis anlehnend zeige ich zunächst Gebietskarten der zwei frequenten schwachen Verben machen und sagen, danach die der irregulären beziehungsweise starken Verben haben, geben, gehen, kommen, werden, daraufhin die der Modalverben dürfen, können, mögen, müssen, sollen, wollen und abschließend die Gebietskarten des Verbs sein. Mit dieser Auswahl versuche ich zu überprüfen, ob sich für die Rückkehr des Präteritums eine ähnliche Hierarchie erkennen lässt wie für den Erhalt, d. h. ob sich die Verbgruppen hinsichtlich der arealen Ausbreitung voneinander unterscheiden. Dabei wurden Kreisdiagrammkarten mit graphischer Skalierung der Ortspunkte gewählt, bei der Orte mit mehr Belegen größer dargestellt werden als Orte mit weniger. Ein stringenter Vergleich zwischen SSA und REDI+FLARS (dt.) ist eigentlich nur für das Badischalemannische möglich, da REDI+FLARS (dt.) im Gegensatz zum SSA nicht das gesamte Alemannische abdeckt. Ich gehe daher explizit auf die Verteilung im vergleichbaren Untersuchungsgebiet (also im Badischalemannischen) ein. Dennoch präsentiere ich die Karten des gesamten Untersuchungsgebietes des SSA, um einen umfassenden Überblick über die areale Verteilung in diesem Korpus zu geben.

Am Ende dieses Unterkapitels fasse ich die wichtigsten Ergebnisse zur arealen Verteilung zusammen. 


\section{Schwache Verben: machen und sagen}

Die Verben machen und sagen ${ }^{14}$ sind im Untersuchungskorpus sehr frequent und finden sich entsprechend in den meisten Ortspunkten in einer einfachen Vergangenheitsform. Machen ist im SSA in 213 von 260 und in REDI+FLARS (dt.) in allen 46 Aufnahmeorten belegt. Mit 2046 Belegen im SSA kommt machen in einem Tempus der einfachen Vergangenheit pro Ort durchschnittlich 9,6-mal vor; in REDI+FLARS (dt.) sind es bei 1019 Belegen im Schnitt 22,2 Belege pro Ort. Sagen ist im SSA in 229 von 260 und in REDI+FLARS (dt.) ebenfalls in allen 46 Ortspunkten zu finden. Bei 3730 Belegen im SSA und 1999 in REDI+FLARS (dt.) entfallen auf jeden Untersuchungsort im Durchschnitt 16,3 bzw. 43,5 Belege von sagen in einem Tempus der einfachen Vergangenheit. Aufgrund der unterschiedlichen Längen der Aufnahmen ist ein Vergleich der durchschnittlichen Belegzahl pro Ort nicht zielführend. Die Zahlen sollen nur einen Einblick in die Datengrundlage geben, auf die sich die Darstellung der jeweiligen Orte stützt. Eine genauere Gegenüberstellung der relativen Frequenzen von Präteritum und Perfekt wird in Kap. 3.1.5 gegeben.

14 Für die Bezeichnung der Verbtypes werden in dieser Arbeit immer die standarddeutschen Entsprechungen verwendet. 
Die nachfolgenden Karten 6 und 7 zeigen die Verteilung des Verbs machen in einfachen Vergangenheitstempora im Korpus SSA und im Korpus REDI+FLARS (dt.). Rote Punkte symbolisieren das Perfekt; blaue das Präteritum.
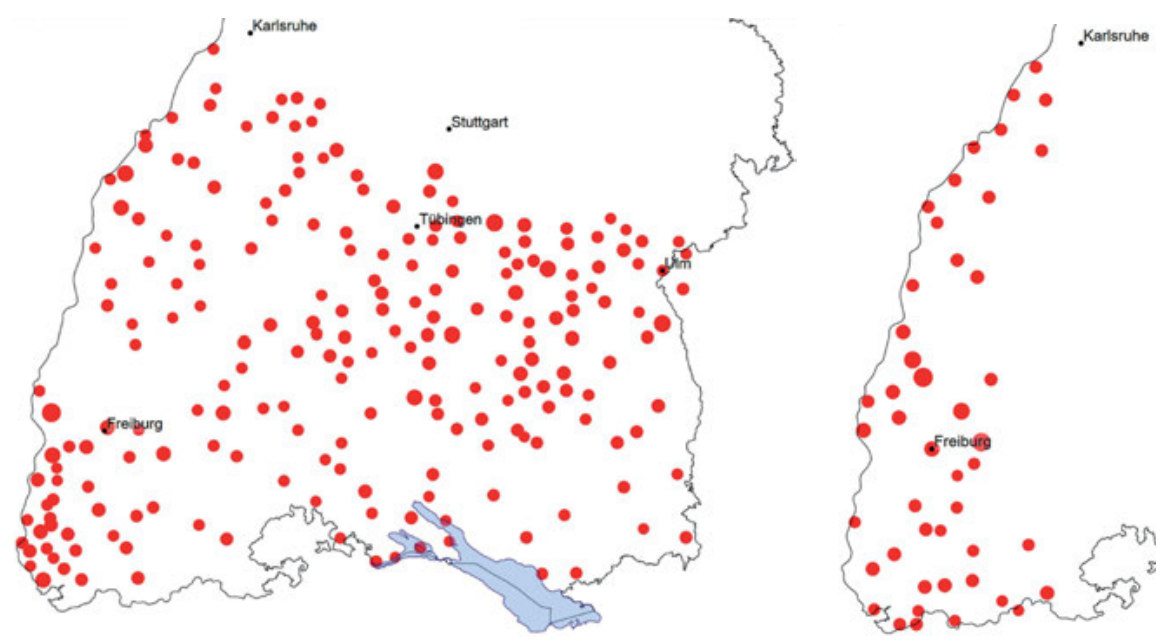

Karte 6 und 7: Areale Verteilung des Verbs machen in einfachen Vergangenheitstempora im SSA [links] und in REDI+FLARS (dt.) [rechts]

Der Vergleich der arealen Verteilung zwischen dem Korpus SSA und dem Korpus REDI+FLARS (dt.) zeigt keine Veränderung in der Präteritum-Perfektverwendung. In beiden Untersuchungszeiträumen (1974-1985 beziehungsweise 20072013) wird ausschließlich Perfekt gebraucht. 
Ein beinahe identisches Bild ist beim Vergleich der beiden Untersuchungszeiträume für das Verb sagen zu sehen. Die nachfolgenden Karten 8 und 9 zeigen die areale Ausbreitung des Verbs sagen in den beiden Untersuchungszeiträumen.
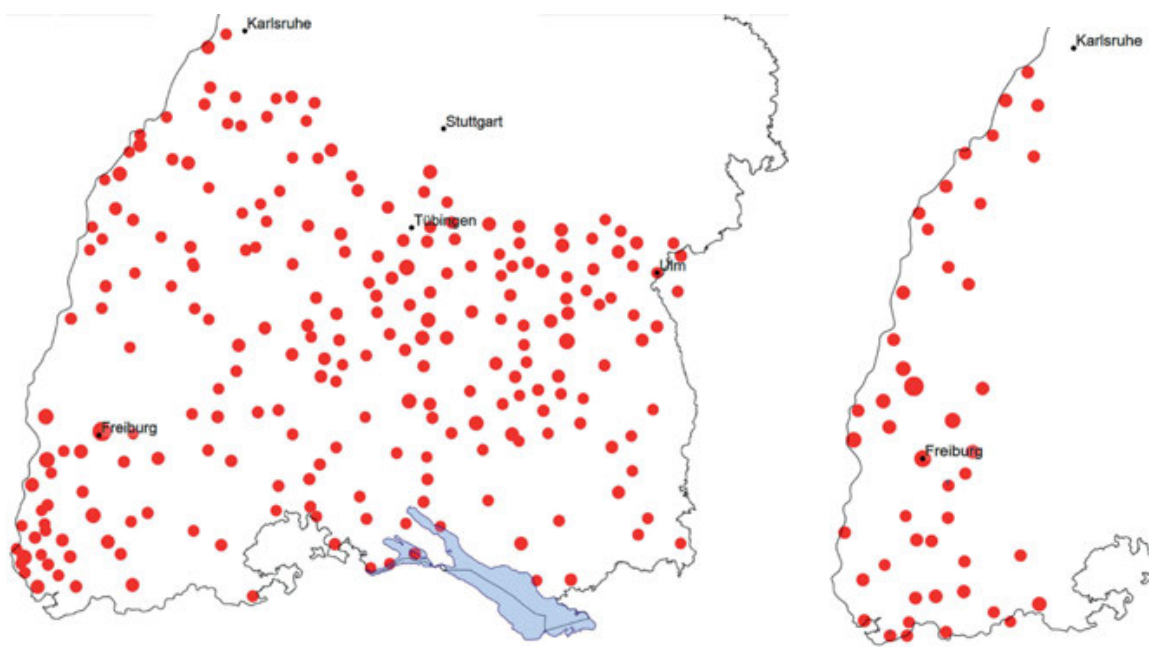

Karte 8 und 9: Areale Verteilung des Verbs sagen in einfachen Vergangenheitstempora im SSA [links] und in REDI+FLARS (dt.) [rechts]

Beim Verb sagen zeigt sich fast keine Veränderung. Während im SSA in keinem Ortspunkt eine Präteritumform nachgewiesen werden kann, sind es in REDI+FLARS (dt.) lediglich zwei Orte. In Herten stehen einer Präteritumform 29 Perfektformen gegenüber. Bei der Präteritumform handelt es sich um eine nichtapokopierte 3. Person-Singular-Form (aber dennoch in dialektaler Sprechweise). Auf die eine Präteritumform (apokopierte 3. Person-Plural-Form) in Oberried kommen 15 Perfekt-Belege. Neben den hier gelisteten Perfekt- und PräteritumBelegen finden sich in den Untersuchungskorpora vereinzelt Belege der 3. Person Singular von sagen, bei denen nicht ersichtlich ist, ob es sich um apokopierte Präteritum- oder um Präsensformen mit Vergangenheitsbezug handelt.

Angesichts dieser Ergebnisse kann weiterhin von einem Fehlen des Präteritums bei schwachen Verben wie machen und sagen im Badischalemannischen Deutschlands ausgegangen werden. 


\section{Irreguläre und starke Verben: haben, geben, gehen, kommen und werden haben}

Die Karten 10 und 11 illustrieren die areale Verteilung des irregulären Vollverbs haben im älteren (1974-1985) und im jüngeren (2007-2013) Untersuchungszeitraum.
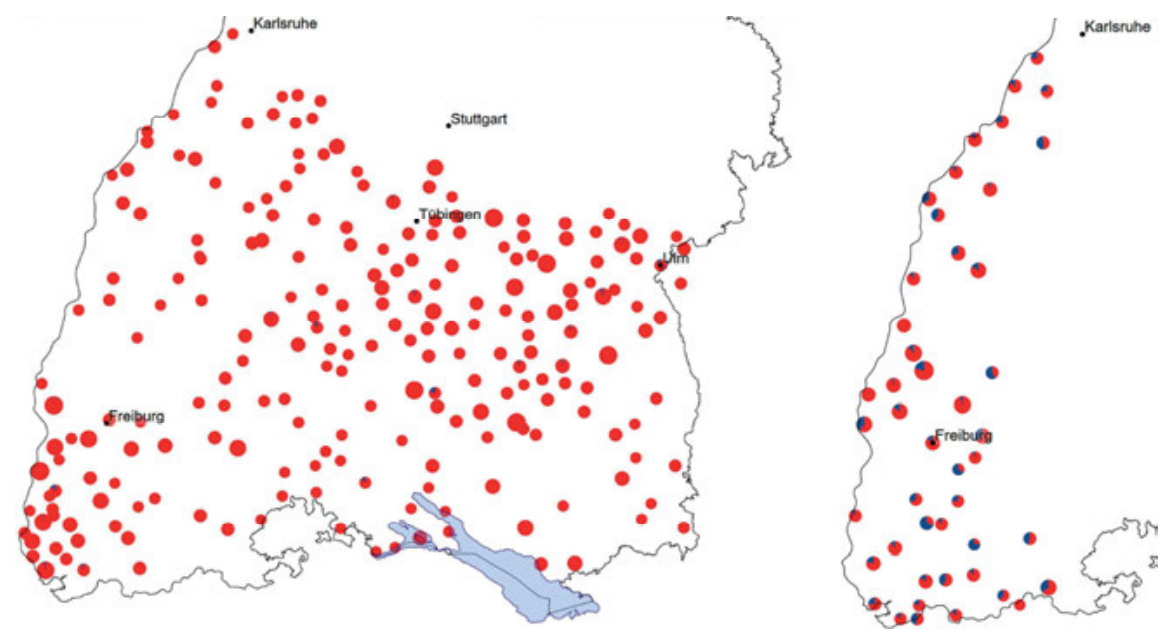

Karte 10 und 11: Areale Verteilung des Vollverbs haben in einfachen Vergangenheitstempora im SSA [links] und in REDI+FLARS (dt.) [rechts]

Die beiden Tempusformen von haben kommen im Korpus SSA in 223 von 260 Orten vor. Bei 3942 Belegen insgesamt sind es durchschnittlich 17,7 Belege pro Ortspunkt. Bei der arealen Verteilung herrscht das Perfekt deutlich vor. Präteritalformen finden sich dagegen nur vereinzelt. Lediglich in 17 Orten sind Präteritalformen neben den Perfektformen zu finden. Dabei macht das Perfekt in jedem Ort die Mehrzahl der Belege aus. Im Niederalemannischen ist überhaupt kein Präteritum zu finden; im Mittel- und Hochalemannischen sind es fünf Ortspunkte, in denen Präteritalformen gebraucht werden. Es gibt also keine auffälligen Unterschiede im Präteritumgebrauch innerhalb des Alemannischen Deutschlands.

In REDI+FLARS (dt.) machen Perfektbildungen ebenfalls den Großteil der Belege aus. In allen 46 Untersuchungsorten ist mindestens eine der beiden Formen zu finden. Auf diese verteilen sich 1593 Belege, d. h. im Durchschnitt 34,6 Belege pro Ort. Südöstlich von Freiburg ist das Präteritumaufkommen etwas höher, nordwestlich etwas geringer. Abgesehen davon ist eine Region, in der das 
Präteritum besonders häufig verwendet wird, nur schwer auszumachen. In drei Ortspunkten kommt nur das Perfekt vor, in 43 beide Tempora. Dabei überwiegt in fünf dieser Orte das Präteritum und in 38 das Perfekt. Auch in Freiburg machen Perfektbildungen die Mehrheit der Belege aus. Elf Sprecher äußern dort sieben Präteritum- und 42 Perfektformen.

Der Vergleich der arealen Ausbreitung zwischen dem ersten und dem zweiten Untersuchungszeitraum zeigt eine deutliche Zunahme der Orte, in denen Präteritalformen zu finden sind, und eine leichte Zunahme der Orte, in denen die Präteritalformen überwiegen. Die Orte, in denen das Perfekt des Vollverbs haben öfter gebildet wird als das Präteritum, machen aber nach wie vor den Großteil aus.

\section{geben}

Die Karten 12 und 13 zeigen die areale Ausbreitung der Perfekt- und Präteritalformen in den Korpora SSA und REDI+FLARS (dt.) für das Verb geben.
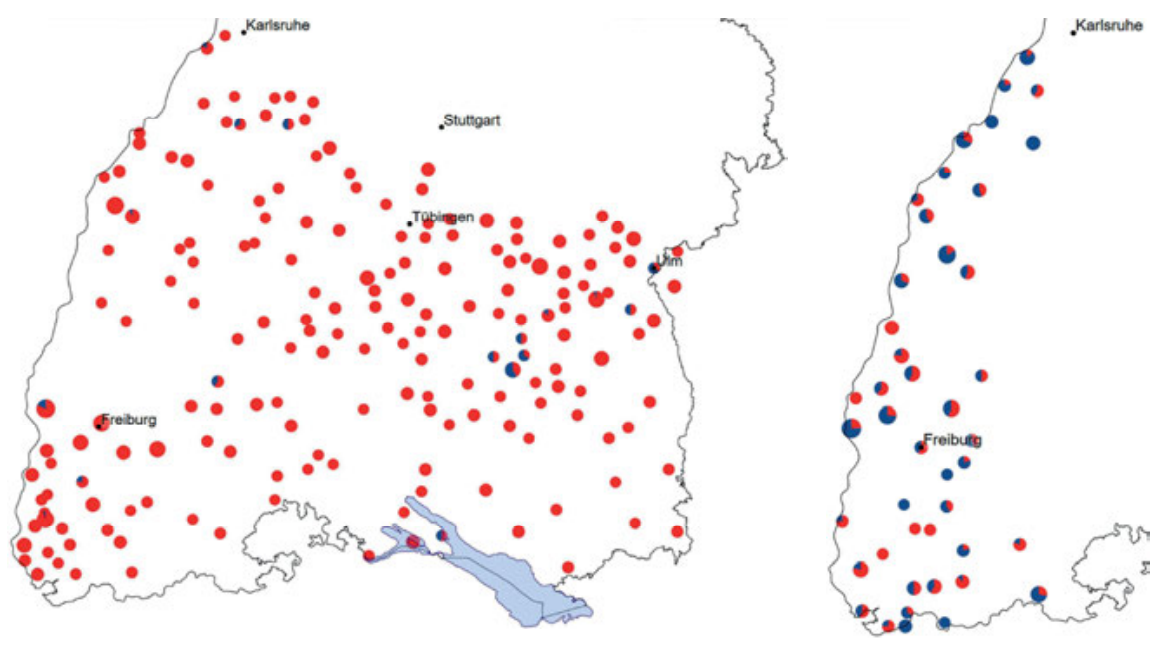

Karte 12 und 13: Areale Verteilung des Verbs geben in einfachen Vergangenheitstempora im SSA [links] und in REDI+FLARS (dt.) [rechts]

Im SSA entfallen 890 Belege auf 186 der 260 Orte, in denen mindestens eine der beiden Formen nachweisbar ist. Auf jeden Untersuchungsort kommen daher durchschnittlich 4,8 Belege. Das Perfekt macht die große Mehrzahl aus. Während in 169 Orten nur Perfekt verwendet wird, gibt es keinen Ort, in dem geben nur im Präteritum gebildet wird. In den 17 Orten, in denen beide Tempora zu finden sind, 
gibt es in drei mehr Präteritum als Perfekt, in neun mehr Perfekt als Präteritum und in fünf gleich viele Präteritum- und Perfektformen. Die drei Orte, in denen mehr Präteritalformen zu finden waren, sind Altheim im Landkreis Biberach, Daugendorf und Ulm. In Altheim äußert ein Sprecher mit ,kommunikationsorientiertem' Beruf sieben Präterital- und eine Perfektform. In Daugendorf und Ulm gebraucht jeweils ein Sprecher zwei Präterital- und eine Perfektform. Der Großteil an Präteritalformen in diesen Orten kann also womöglich durch die geringe Sprecher- und Belegzahl erklärt werden. Im Badischalemannischen sind in sechs Orten Präteritumformen zu finden. Auch hier gibt es keine auffälligen Unterschiede im Präteritumgebrauch innerhalb des Untersuchungsgebiets.

In REDI+FLARS (dt.) lassen sich Perfekt- und/oder Präteritumformen des Verbs geben in 44 der 46 Untersuchungsorte nachweisen. Auf diese verteilen sich 395 Belege. Das entspricht einer durchschnittlichen Belegzahl von 8,9 pro Ort. Die Perfekt-Präteritum-Verteilung ist dabei fast ausgeglichen. In sechs Orten kommt nur Präteritum vor, in sechs nur Perfekt. In 32 Orten können beide Formen nachgewiesen werden. In 16 dieser Orte ist der Anteil der Präteritumformen höher und in 14 der Anteil der Perfektformen. In zwei ist der Anteil beider Formen gleich hoch. Auch beim Verb geben gibt es in Freiburg keinen höheren Präteritalgebrauch bei vier Sprechern, die drei Präterial- und vier Perfektformen äußern.

Im SSA macht mit Ausnahme von einzelnen Belegen das Perfekt noch die große Mehrheit der Belege aus. Zum Korpus REDI+FLARS (dt.) ist eine deutliche Zunahme an Orten, in denen Präteritalformen gebraucht werden, und eine deutliche Zunahme an Orten, in denen nur Präteritum oder überwiegend Präteritum verwendet wird, zu verzeichnen. 


\section{gehen}

Die Karten 14 und 15 verdeutlichen die areale Ausbreitung der Perfekt- und Präteritumbildungen des Verbs gehen in den Korpora SSA und REDI+FLARS (dt.).
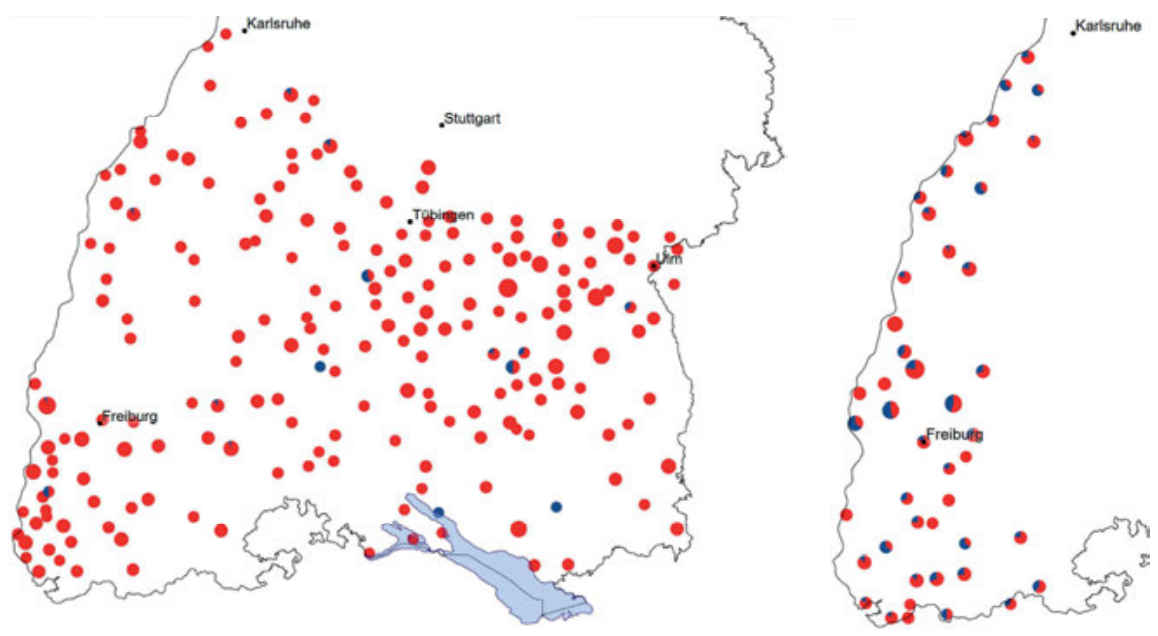

Karte 14 und 15: Areale Verteilung des Verbs gehen in einfachen Vergangenheitstempora im SSA [links] und in REDI-FLARS (dt.) [rechts]

Das Verb gehen ist im Korpus SSA in 203 von 260 Orten zu finden. 1164 Belege insgesamt bedeuten 5,7 Belege durchschnittlich pro Erhebungsort. Als Tempus der einfachen Vergangenheit wird vorwiegend Perfekt gebraucht. Dies zeigt sich bei drei Orten, in denen ausschließlich Präteritumformen zu finden sind. Demgegenüber können in 187 Orten nur Perfektbildungen nachgewiesen werden. In 13 Orten gibt es im Untersuchungskorpus beide Formen, wobei in zehn das Perfekt und in einem das Präteritum die Mehrheit ausmacht und in zwei Orten beide Formen gleich häufig gebraucht werden. Abgesehen von Ravensburg, wo ausschließlich Präteritalformen gebraucht werden, wird in den größeren Städten nur Perfekt verwendet. Neben Ravensburg ist auch in Überlingen und Wellendingen ausschließlich das Präteritum zu finden. Allerdings verwendet in diesen drei Orten jeweils lediglich ein Sprecher eine Präteritumform. Die markanten Präteritumverwendungen in diesen Orten sind also wahrscheinlich durch die geringe Belegzahl zu erklären. Im Badischalemannischen kommt in drei Orten neben Perfekt auch ein Präteritum des Verbs gehen vor. Abgesehen von den drei Orten, in denen nur Präteritum verkommt, gibt es keine auffälligen Unterschiede im Präteritumgebrauch innerhalb des Alemannischen Deutschlands. 
Im Korpus REDI+FLARS (dt.) lässt sich in 45 der 46 Untersuchungsorte mindestens eines der beiden Vergangenheitstempora des Verbs gehen nachweisen. Bei 514 Belegen insgesamt entfallen im Durchschnitt auf jeden Ort 11,4 Belege. Die Mehrzahl macht dabei das Perfekt aus: In neun Untersuchungsorten kommt ausschließlich Perfekt vor; in keinem Ort ausschließlich Präteritum. Bei den 36 Orten, in denen beide Tempora zu finden sind, ist das Präteritum siebenmal das häufigere Tempus und das Perfekt 28-mal. In einem Ort werden die beiden Tempora gleich oft gebildet. Auch in Freiburg gibt es im Untersuchungskorpus mehr Perfekt als Präteritum. Fünf Sprecher produzieren zwei Präterital- und neun Perfektformen.

Es ist zwischen den beiden Untersuchungszeiträumen ein markanter Anstieg an Orten zu erkennen, in denen Präteritum gebraucht wird. Hinsichtlich der Anzahl an Orten, in denen nur Präteritum nachgewiesen wurde, hat die Zahl zwar abgenommen. Dies liegt aber an den unterschiedlich großen Untersuchungsgebieten in den beiden Korpora. Im Badischalemannischen gibt es in beiden Korpora keinen Ort, in dem nur Präteritum zu finden war.

\section{kommen}

Die nachfolgenden Karten 16 und 17 präsentieren die areale Verteilung der Perfekt- und Präteritumformen des Verbs kommen in den zwei Untersuchungskorpora.
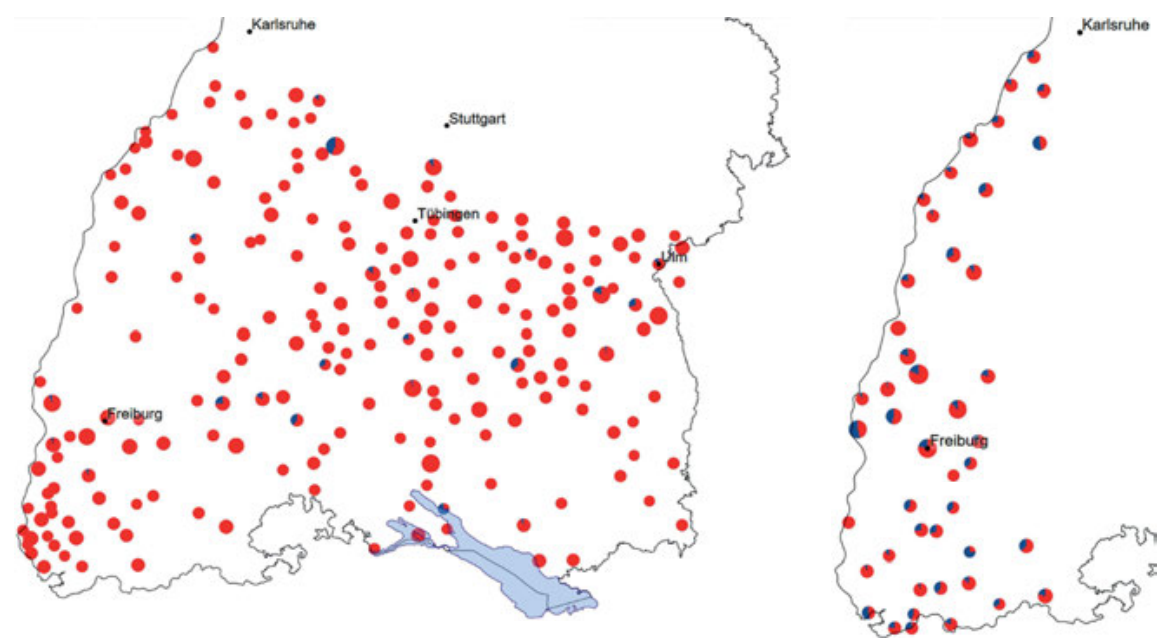

Karte 16 und 17: Areale Verteilung des Verbs kommen in einfachen Vergangenheitstempora im SSA [links] und in REDI+FLARS (dt.) [rechts] 
Im Korpus SSA treten die beiden Tempusformen von kommen in 211 von 260 Orten auf. Bei 1966 Belegen insgesamt sind es durchschnittlich 9,3 Belege pro Ortspunkt. Bei der arealen Verteilung ist der Anteil der Perfektformen deutlich höher. Präteritalformen finden sich dagegen nur vereinzelt. In keinem Ort wird nur Präteritum verwendet, in 188 dagegen ausschließlich Perfekt. In den 23 Orten, in denen beide Tempora vorkommen, macht das Perfekt den Großteil aus. Auch in den größeren Städten sind entweder nur Perfekt- oder Perfekt- und Präteritumformen zu finden. Im Badischalemannischen wird in vier Orten neben Perfekt auch Präteritum gebraucht. Im Südwesten kommt das Präteritum fast gar nicht vor. Im badischalemannischen Teilgebiet des SSA wird die Präteritumform von kommen also etwas seltener verwendet als im restlichen Untersuchungsgebiet.

In REDI+FLARS (dt.) machen Perfektbildungen ebenfalls die Mehrheit der Belege aus. In 45 der 46 Untersuchungsorte ist mindestens eine der beiden Formen zu finden. Auf diese verteilen sich 972 Belege, d. h. im Durchschnitt 21,6 Belege pro Ort. In 42 Ortspunkten kommen beide Tempora vor, in drei nur das Perfekt, in keinem nur Präteritum. In den Orten, in denen beide Tempora verwendet werden, überwiegt in drei dieser Orte das Präteritum und in 39 das Perfekt. Auch in Freiburg machen Perfektbildungen die große Mehrheit der Belege aus. Zwölf Sprecher äußern dort 13 Präteritum- und 48 Perfektformen.

Der Vergleich der arealen Ausbreitung zwischen dem ersten und dem zweiten Untersuchungszeitraum zeigt einen deutlichen Zuwachs der Orte, in denen Präteritalformen $\mathrm{zu}$ finden sind, aber kaum eine Zunahme an Orten, in denen mehr Präteritum als Perfekt gebraucht wird. Auch im Korpus REDI+FLARS (dt.) sind es mehrheitlich Perfektbildungen. 


\section{werden}

In den Karten 18 und 19 ist die areale Ausbreitung der Perfekt- und Präteritumbildungen des Verbs werden in den Korpora SSA und REDI+FLARS (dt.) zu sehen.
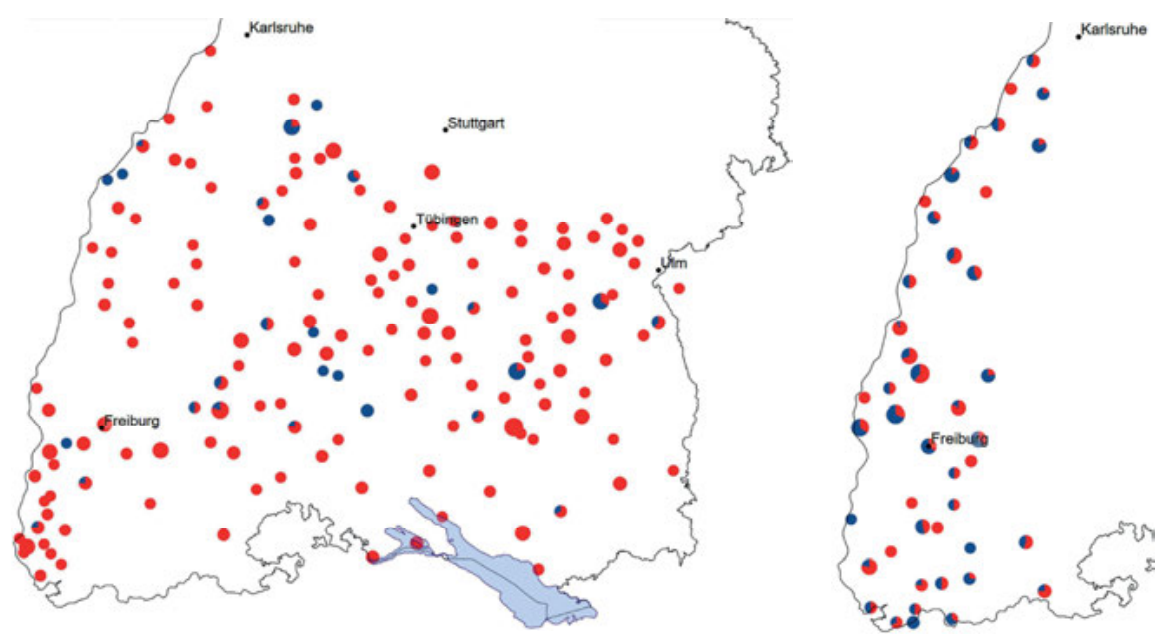

Karte 18 und 19: Areale Verteilung des Verbs werden in einfachen Vergangenheitstempora im SSA [links] und in REDI+FLARS (dt.) [rechts]

439 Belege des Verbs werden im Perfekt oder Präteritum verteilen sich im Korpus SSA auf 157 der insgesamt 260 Untersuchungsorte. Dementsprechend entfallen auf jeden Erhebungsort im Durchschnitt 2,8 Belege. Dabei überwiegt das Perfekt deutlich. Zehn Orten, in denen nur Präteritum gebraucht wird, stehen 130 Orte gegenüber, in denen nur Perfekt verwendet wird. In 17 Orten kommen beide Tempora vor. In vier dieser Orte überwiegt der Anteil an Präterita, in elf Orten der Anteil der Perfekte und in zwei Orten sind es von beiden Tempora gleich viel. Im Badischalemannischen ist in drei Orten nur Präteritum zu finden; in fünf beide Tempora (davon in zwei Gebieten genauso viele Perfekte wie Präterita und in drei mehr Perfekt- als Präteritumformen). In den drei Orten, in denen ausschließlich das Präteritum des Verbs werden gebraucht wird, ist jeweils nur ein Beleg nachweisbar. Diese Auffälligkeiten gehen also vermutlich auf die geringe Belegzahl zurück. Es lässt sich also kein auffälliger Unterschied im Präteritumgebrauch innerhalb des Alemannischen Deutschlands feststellen.

In 44 der 46 Untersuchungsorte des Korpus REDI+FLARS (dt.) ist das Verb werden in einem Tempus der einfachen Vergangenheit belegt. 340 Belege insgesamt entsprechen durchschnittlich 7,7 Belegen pro Ort. Mit acht Orten, in denen 
nur Perfekt vorherrscht, gegenüber drei, in denen nur Präteritum nachgewiesen wurde, ist der Anteil der Perfektbildungen dabei etwas höher als der der Präteritumbildungen. In 33 Orten waren beide Tempora zu finden: In 13 überwog der Anteil der Präterita, in zwölf der Anteil der Perfekte und in acht war der Anteil beider Tempora gleich hoch. In Freiburg kommt bei sieben Sprechern, die neun Präteritum- und fünf Perfektformen bilden, das Präteritum häufiger vor als das Perfekt.

Es lässt sich also ein deutlicher Anstieg der Orte erkennen, in denen Präteritum gebraucht wird, und eine geringe Zunahme an Orten, in denen Präteritum den größeren Anteil an Vergangenheitstempora vorweist.

\section{Modalverben: dürfen, können, mögen, müssen, sollen und wollen}

In meinen Daten zeigte sich, dass bei den Modalverben sollen und wollen Indikativ Präteritum und Konjunktiv II wie im Standarddeutschen homophon sind. Für die Identifizierung der Belege als Indikativ Präteritum habe ich für jeden Beleg überprüft, ob ein Vergangenheitsbezug besteht. Wenn dieser gegeben war, wurde der jeweilige Beleg als Indikativ Präteritum eingestuft, andernfalls als Konjunktiv II. In Beispiel (26) ist eine Verwendung der Verbform sollt zu sehen, die keinen Vergangenheitsbezug hat, in Beispiel (27) eine Verwendung mit Vergangenheitsbezug. Dementsprechend wurde der Beleg in Beispiel (26) als Konjunktiv II und der Beleg in Beispiel (27) als Indikativ Präteritum bewertet.

(26) Achern (REDI+FLARS (dt.))

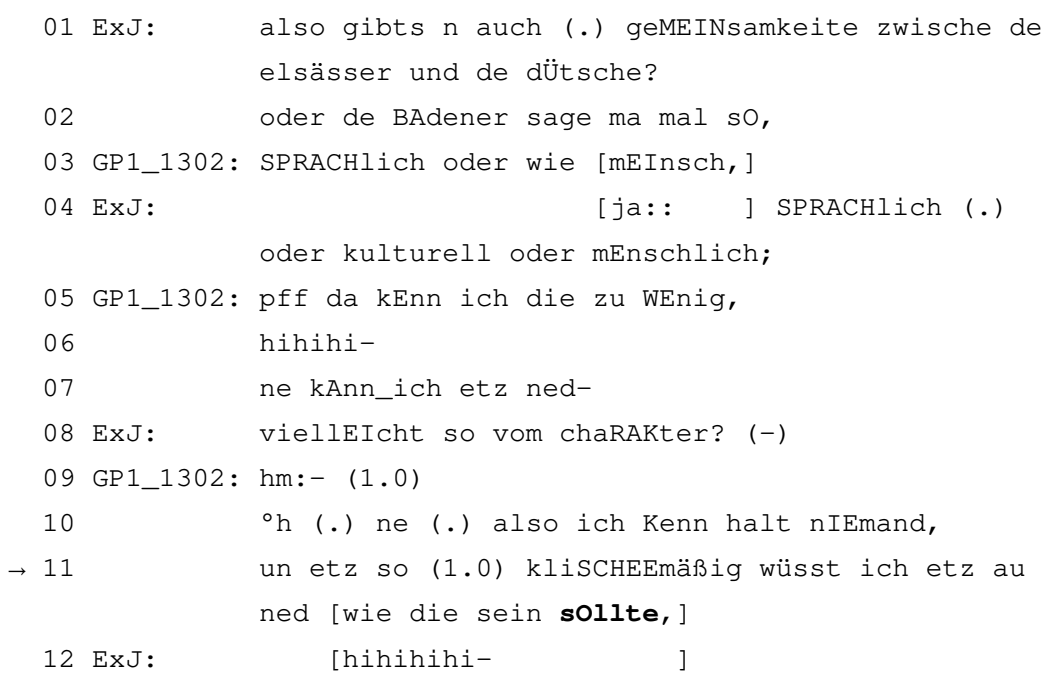


Die Interviewerin ExJ fragt hier, ob es Gemeinsamkeiten zwischen Elsässern und Deutschen beziehungsweise Badenern gibt (Z. 01-02). Nach der Frage der Sprecherin GP1_1302, welchen Aspekt die Interviewerin meine, (Z. 03) und der Beantwortung dieser Frage (Z. 04) antwortet GP1_1302 ab Zeile 05, dass sie zu wenige Elsässer kenne. In Zeile 11 schließt sie ihre Antwortsequenz ab, indem sie angibt, sie kenne auch keine Klischees darüber, „wie die sein sOllte“. Diese Äußerung hat keinen Vergangenheitsbezug, sondern vermittelt einen hypothetischen Fall. Es handelt sich daher um einen Konjunktiv II und nicht um ein Indikativ Präteritum.

(27) Opfingen (REDI+FLARS (dt.))

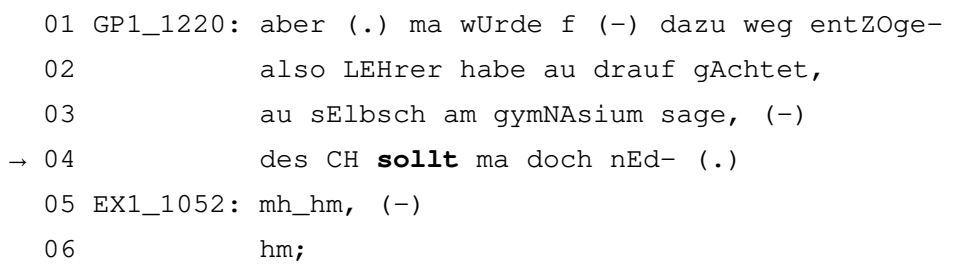

Im Vorfeld dieses Ausschnitts erzählt der Sprecher GP1_1220, dass er das „CH“ (Z. 04) seltener verwende als früher. Er begründet dies ab Zeile 01 damit, dass Schülern der Dialekt abgewöhnt wurde. Er spezifiziert dies in Zeile 04, indem er die Vorgabe der Lehrer wiedergibt: „des CH sollt ma doch nEd“. Hierbei ist ein Vergangenheitsbezug erkennbar, da er eine Episode aus der Vergangenheit erzählt, zu der auch die Äußerung in Zeile 04 zu zählen ist. 


\section{dürfen}

Die Karten 20 und 21 illustrieren die areale Verteilung des Modalverbs dürfen im älteren (1974-1985) und im jüngeren (2007-2013) Untersuchungszeitraum.
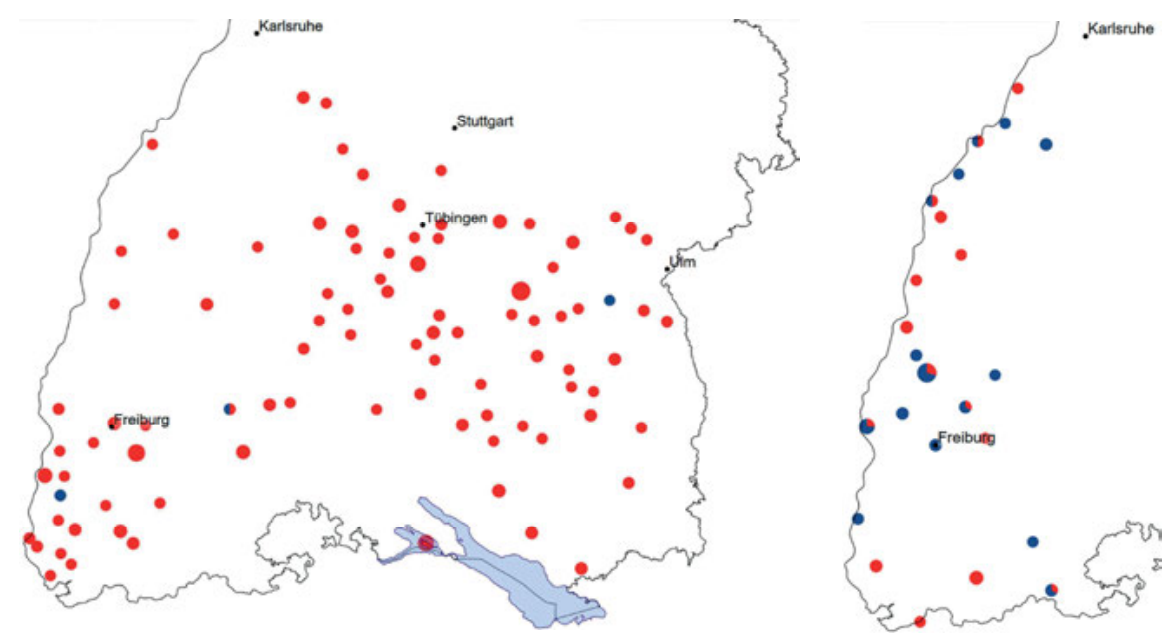

Karte 20 und 21: Areale Verteilung des Verbs dürfen in einfachen Vergangenheitstempora im SSA [links] und in REDI+FLARS (dt.) [rechts]

Die beiden Tempusformen von dürfen kommen im Korpus SSA in 91 von 260 Orten vor. Bei 199 Belegen insgesamt sind es durchschnittlich 2,2 Belege pro Ortspunkt. Bei der arealen Verteilung macht das Perfekt fast alle Belege aus. Präteritalformen finden sich dagegen nur vereinzelt. In 88 Erhebungsorten sind ausschließlich Perfektformen zu finden, in zwei nur Präteritumformen und in einem Ort sind beide Tempusformen gleich oft belegt. In diesen drei Orten mit Präteritum produziert jeweils nur ein Sprecher alle Präteritalformen. In Altheim bei Ulm und Vöhrenbach kommt je ein Präteritum vor, in Zunzingen zwei Formen. Zunzingen ist auch der einzige Ort im Badischalemannischen, in dem Präteritum nachgewiesen werden kann. Es gibt also keine auffälligen Unterschiede im Präteritumgebrauch innerhalb des Alemannischen Deutschlands.

In REDI+FLARS (dt.) ist der Anteil an Perfekt- und an Präteritumbildungen dagegen fast gleich hoch. In 24 von 46 Untersuchungsorten ist mindestens eine der beiden Formen zu finden. Auf diese verteilen sich 67 Belege, d. h. im Durchschnitt 5,9 Belege pro Ort. In neun Ortspunkten kommt nur das Perfekt, in neun weiteren nur das Präteritum vor, in sechs beide Tempora. Dabei überwiegt in vier dieser Orte das Präteritum und in zwei keines der beiden Tempora. In Freiburg 
sind alle Belege Präteritumbildungen (bei einem Sprecher, der drei Belege produziert). Im Mittelalemannischen lässt sich eine Häufung an Präteritumformen erfassen.

Der Vergleich der arealen Ausbreitung zwischen dem ersten und dem zweiten Untersuchungszeitraum zeigt eine deutliche Zunahme der Orte, in denen Präteritalformen zu finden sind, und eine deutliche Zunahme der Orte, in denen die Präteritalformen überwiegen, sodass im jüngeren Korpus der Anteil an Präteritum- und Perfektformen etwa gleich hoch ist.

\section{können}

Die Karten 22 und 23 zeigen die areale Ausbreitung der Perfekt- und Präteritalformen in den Korpora SSA und REDI+FLARS (dt.) für das Modalverb können.
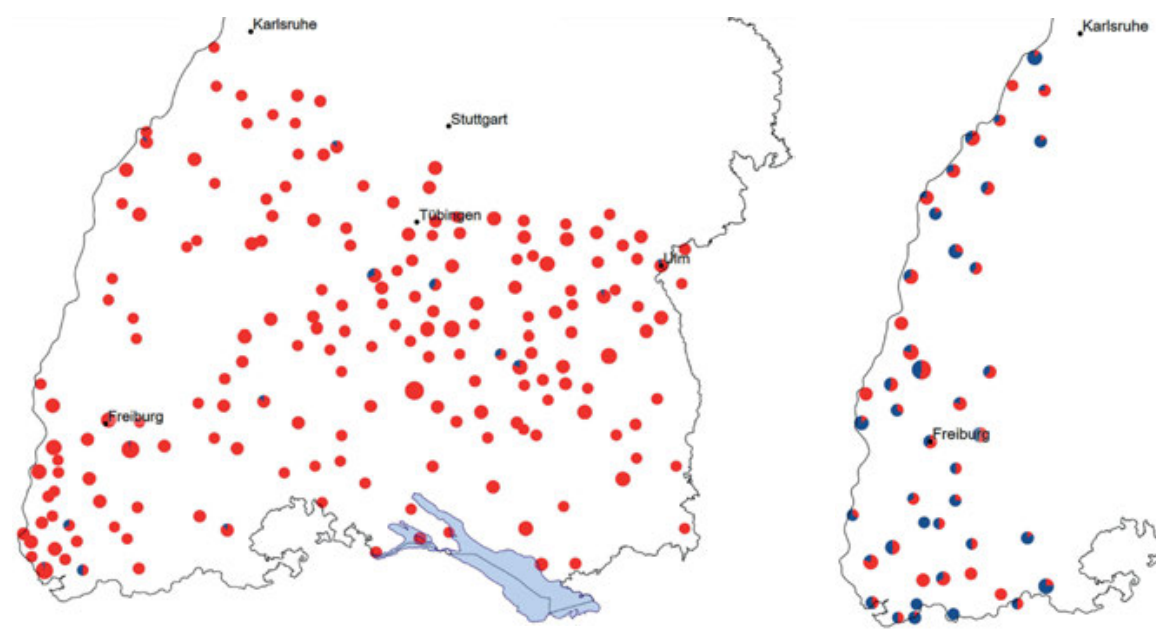

Karte 22 und 23: Areale Verteilung des Verbs können in einfachen Vergangenheitstempora im SSA [links] und in REDI+FLARS (dt.) [rechts]

Im SSA entfallen 991 Belege auf 182 der 260 Orte, in denen mindestens eine der beiden Formen nachweisbar ist. Auf jeden Untersuchungsort kommen daher durchschnittlich 5,4 Belege. Das Perfekt macht die große Mehrzahl aus. Während in 168 Orten nur Perfekt verwendet wird, gibt es keinen Ort, in dem nur Präteritum gebraucht wird. In den 14 Orten, in denen beide Tempora zu finden sind, gibt es in 13 mehr Perfekt als Präteritum und in einem gleich viele Präteritum- und Perfektformen. Abgesehen von Ulm, wo auf ein Präteritum neun Perfektbildungen kommen, ist in den größeren Städten gar kein Präteritum zu finden. Im 
Badischalemannischen sind in fünf Orten Präteritumformen zu finden. Während in vier dieser Orte das Perfekt die große Mehrzahl der Belege darstellt, ist der Anteil der beiden Tempora nur in Adelhausen ausgeglichen (eine Präteritum- und eine Perfektform). Innerhalb des Untersuchungsgebiets finden sich daher keine auffälligen Unterschiede im Präteritumgebrauch.

In REDI+FLARS (dt.) lassen sich Perfekt- und/oder Präteritumformen des Verbs können in 45 der 46 Untersuchungsorte nachweisen. Auf diese verteilen sich 392 Belege. Das entspricht einer durchschnittlichen Belegzahl von 8,7 pro Ort. Die Perfekt-Verteilung ist dabei etwas höher als die Präteritum-Verteilung. In drei Orten kommt nur Präteritum vor, in sechs nur Perfekt. In 37 Orten können beide Formen nachgewiesen werden. In 13 dieser Orte ist der Anteil der Präteritumformen, in 18 der Anteil der Perfektformen höher, in sechs ist der Anteil beider Formen gleich hoch. Die grundsätzliche Verteilung spiegelt sich auch in der von Freiburg wider. Dort gibt es bei acht Sprechern, die vier Präterial- und sechs Perfektformen äußern, eine leichte Tendenz zum Perfektgebrauch.

Im SSA macht das Perfekt die große Mehrheit der Belege aus. Zum Korpus REDI+FLARS (dt.) ist eine deutliche Zunahme an Orten, in denen Präteritalformen gebraucht werden, und eine deutliche Zunahme an Orten, in denen nur Präteritum oder überwiegend Präteritum verwendet wird, zu verzeichnen. Im jüngeren Korpus ist die Verteilung der beiden Tempora somit fast ausgeglichen. 


\section{mögen}

Auf den Karten 24 und 25 ist die areale Verteilung des Modalverbs mögen in den Korpora SSA und REDI+FLARS (dt.) zu sehen.
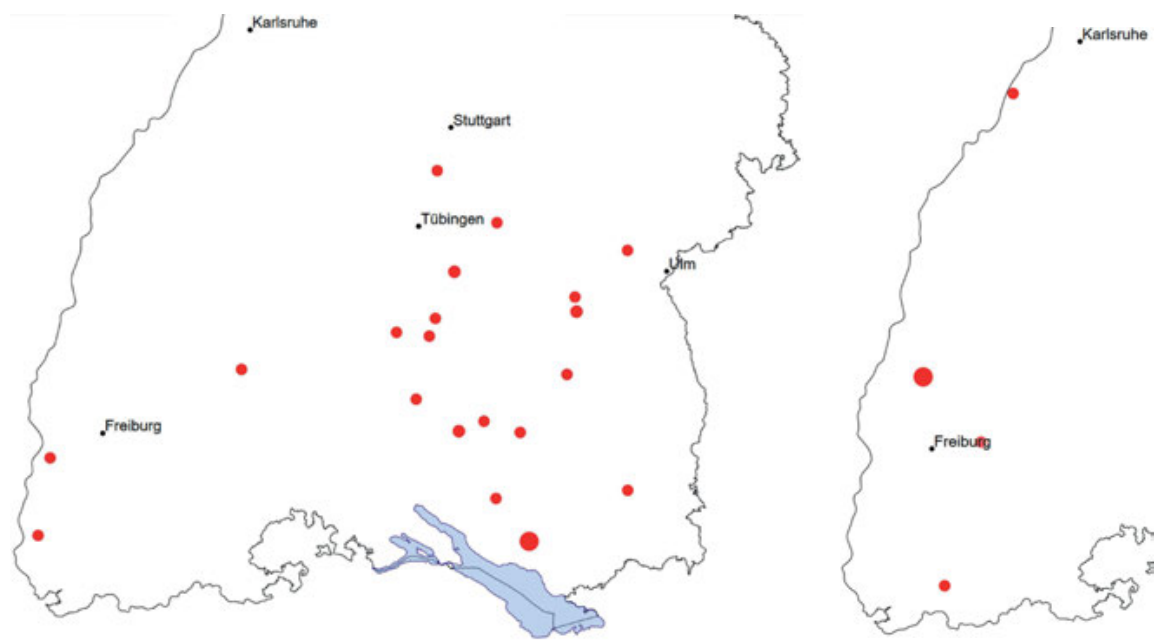

Karte 24 und 25: Areale Verteilung des Verbs mögen in einfachen Vergangenheitstempora im SSA [links] und in REDI+FLARS (dt.) [rechts]

Das Modalverb mögen ist mit 30 Belegen im SSA (verteilt auf 20 Orte) und fünf Belegen in REDI+FLARS (dt.) (verteilt auf vier Orte) extrem selten in einem Vergangenheitstempus belegt. Der Vergleich des älteren mit dem jüngeren Untersuchungszeitraum zeigt keine Veränderung in der Tempusverwendung. In beiden Korpora wird ausschließlich Perfekt verwendet. Aufgrund der geringen Belegzahl sind valide Aussagen hier nicht möglich. So muss unklar bleiben, ob die Präteritalform wegen der geringen Belegzahl nicht nachgewiesen werden konnte oder ob aufgrund der geringen Belegzahl überhaupt kein Präteritum wieder ins Dialektgebiet eingedrungen ist beziehungsweise dem Präteritumschwund Stand gehalten hat. 


\section{müssen}

Die Karten 26 und 27 verdeutlichen die areale Ausbreitung der Perfekt- und Präteritumbildungen des Modalverbs müssen in den Korpora SSA und REDI+FLARS (dt.).
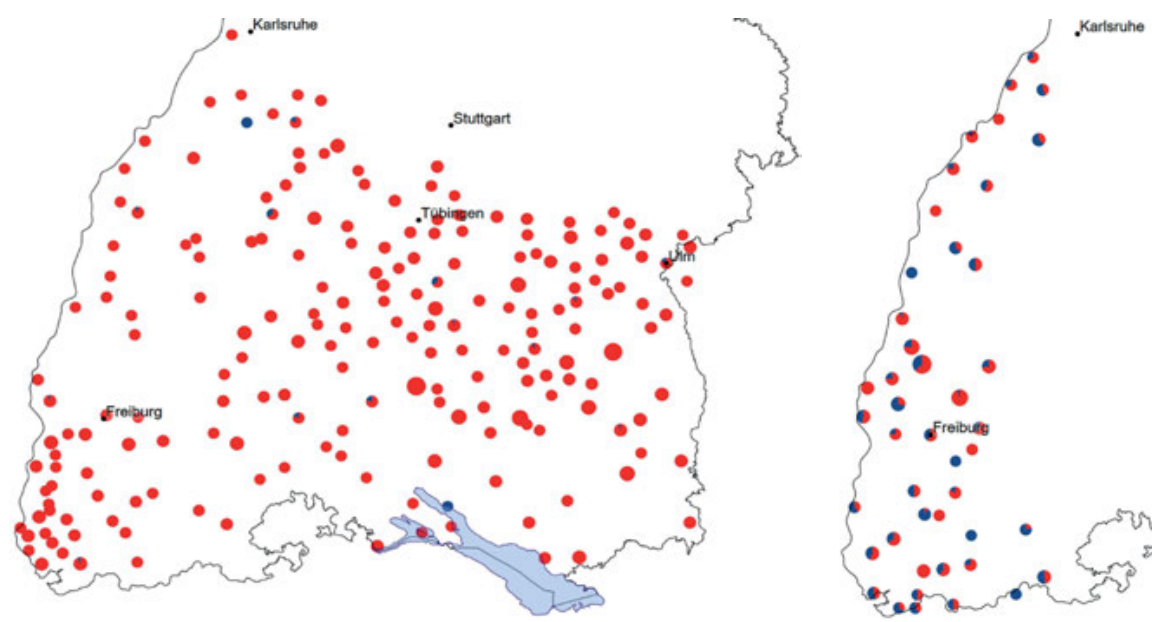

Karte 26 und 27: Areale Verteilung des Verbs müssen in einfachen Vergangenheitstempora im SSA [links] und in REDI+FLARS (dt.) [rechts]

Das Modalverb müssen ist im Korpus SSA in 199 von 260 Orten zu finden. 2123 Belege insgesamt bedeuten 10,7 Belege durchschnittlich pro Erhebungsort. Als Tempus der einfachen Vergangenheit wird fast ausschließlich Perfekt gebraucht. Dies zeigt sich bei nur zwei Orten, in denen ausschließlich Präteritumformen zu finden sind, und 184 Orten, in denen nur Perfektbildungen nachgewiesen werden können. In 13 Orten gibt es im Untersuchungskorpus beide Formen, wobei das Perfekt in diesen Orten die Mehrheit ausmacht. Die beiden Orte, in denen nur Präteritalformen nachgewiesen werden können, sind Loffenau und Überlingen. In beiden Orten verwendet allerdings jeweils ein Sprecher zwei Belege im Präteritum. Die markanten Präteritumverwendungen in diesen Orten sind also wahrscheinlich durch die geringe Belegzahl zu erklären. Im Badischalemannischen kommt in drei Orten neben Perfekt auch ein Präteritum des Modalverbs müssen vor; in einem Ort (Loffenau) nur das Präteritum. Es gibt also keine auffälligen Unterschiede im Präteritumgebrauch innerhalb des Alemannischen Deutschlands. 
Im Korpus REDI+FLARS (dt.) lässt sich in 45 der 46 Untersuchungsorte mindestens eines der beiden Vergangenheitstempora des Modalverbs müssen nachweisen. Bei 478 Belegen insgesamt entfallen im Durchschnitt auf jeden Ort 10,6 Belege. Das Perfekt macht dabei etwas mehr als die Hälfte aus: in sechs Untersuchungsorten kommt ausschließlich das Perfekt vor; in vier nur das Präteritum. Bei den 35 Orten, in denen beide Tempora zu finden sind, ist das Präteritum elfmal das häufigere Tempus und das Perfekt 20-mal. In vier Orten sind die beiden Tempora gleich oft nachweisbar. Auch in Freiburg gibt es im Untersuchungskorpus mehr Perfekt als Präteritum. Zehn Sprecher produzieren sechs Präterital- und zehn Perfektformen.

Zwischen den beiden Untersuchungszeiträumen lässt sich ein markanter Anstieg an Orten erkennen, in denen Präteritum gebraucht wird, sowie an Orten, an denen ausschließlich Präteritum verwendet wird. Während im SSA fast ausschließlich das Perfekt verwendet wird, ist das Verhältnis mittlerweile beinahe ausgeglichen.

\section{sollen}

Die nachfolgenden Karten 28 und 29 präsentieren die areale Verteilung der Perfekt- und Präteritumformen des Modalverbs sollen in den beiden Untersuchungskorpora.
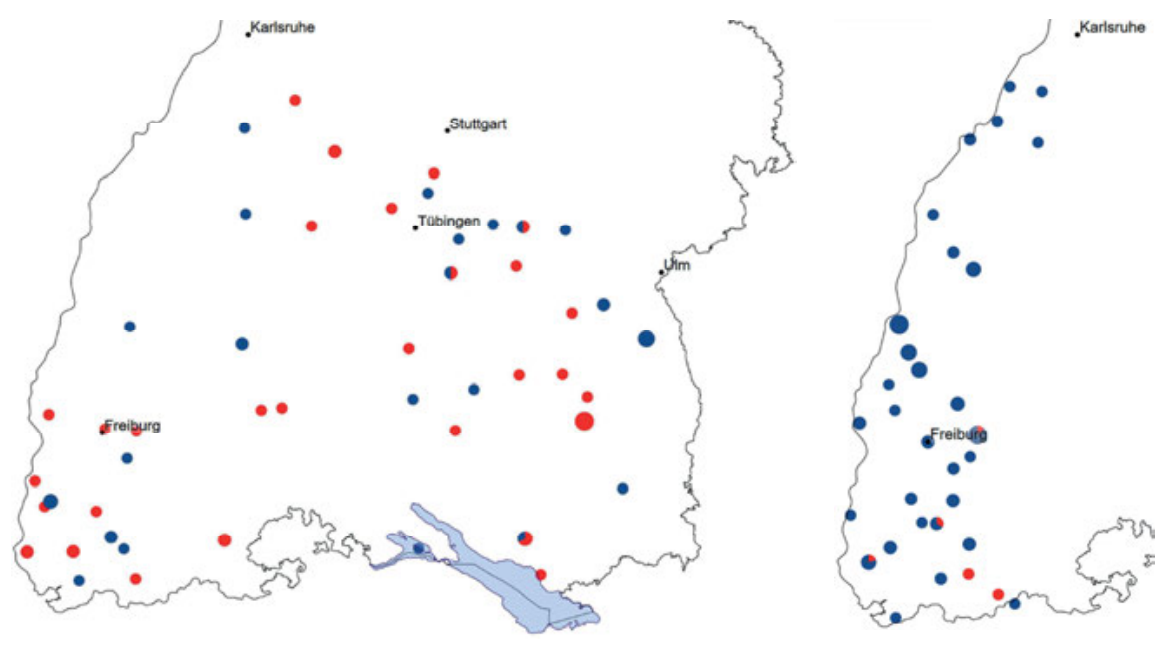

Karte 28 und 29: Areale Verteilung des Verbs sollen in einfachen Vergangenheitstempora im SSA [links] und in REDI+FLARS (dt.) [rechts] 
Die beiden Tempusformen von sollen kommen im Korpus SSA in 48 von 260 Orten vor. Bei 68 Belegen insgesamt sind es durchschnittlich 1,4 Belege pro Ortspunkt. Bei der arealen Verteilung ist der Anteil der Perfektformen etwas höher als der der Präteritumformen. In 19 Orten wird nur Präteritum verwendet, in 26 ausschließlich Perfekt. In einem der drei Orte, in denen beide Tempora vorkommen, macht das Perfekt den Großteil aus; in zwei Orten sind es genauso viele Perfektwie Präteritumformen. Trotz der vielen Orte, in denen nur eine der beiden Tempusformen nachweisbar ist, lässt sich keine geographische Häufung an Perfekt oder Präteritum erkennen. Die Tatsache, dass es so viele Orte mit nur einem der beiden Tempora im Untersuchungskorpus gibt, liegt sicherlich an der durchschnittlich geringen Belegzahl pro Ort. Wahrscheinlich sind die beiden Tempora also über das gesamte Untersuchungsgebiet etwa gleichmäßig verteilt. Im Badischalemannischen wird in acht Orten nur Präteritum gebraucht, in elf nur Perfekt. Ein Vergleich des badischalemannischen Teilgebiets mit dem restlichen Untersuchungsgebiet zeigt keine markanten Unterschiede im Präteritumgebrauch.

In REDI+FLARS (dt.) ist in 21 der 46 Untersuchungsorte mindestens eine der beiden Formen zu finden. Auf diese Orte verteilen sich 41 Belege, d. h. im Durchschnitt 2,0 Belege pro Ort. Das Präteritum macht dabei fast alle Belege aus. In 16 Ortspunkten kommt nur das Präteritum vor, in drei nur das Perfekt, in zwei beide Tempora, wobei in diesen beiden Orten das Präteritum überwiegt. In den beiden Orten, in denen nur Perfekt zu finden ist (Buch und Herrischried), äußert je ein Sprecher nur einen Beleg. Diese Auffälligkeit ist also wahrscheinlich durch die geringe Belegzahl zu erklären.

Der Vergleich der arealen Ausbreitung zwischen dem ersten und dem zweiten Untersuchungszeitraum zeigt einen deutlichen Zuwachs der Orte, in denen Präteritalformen zu finden sind, und der Orte, in denen nur Präteritum gebraucht wird. Während der Anteil der Perfekt-Belege im SSA etwas höher ist, entfallen fast alle Belege in REDI+FLARS (dt.) auf das Präteritum. 


\section{wollen}

In den Karten 30 und 31 ist die areale Ausbreitung der Perfekt- und Präteritumbildungen des Modalverbs wollen in den Korpora SSA und REDI+FLARS (dt.) zu sehen.
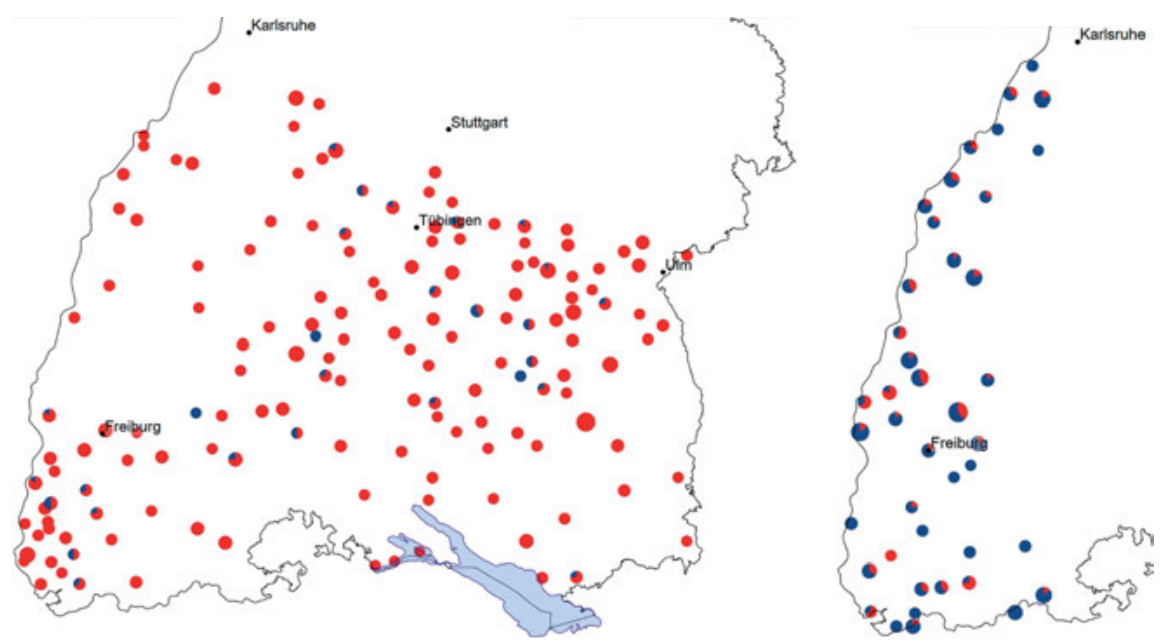

Karte 30 und 31: Areale Verteilung des Verbs wollen in einfachen Vergangenheitstempora im SSA [links] und in REDI+FLARS (dt.) [rechts]

534 Belege des Modalverbs wollen im Perfekt oder Präteritum verteilen sich im Korpus SSA auf 152 der insgesamt 260 Untersuchungsorte. Dementsprechend entfallen auf jeden Erhebungsort im Durchschnitt 3,5 Belege. Dabei überwiegt das Perfekt deutlich, während Präteritum nur vereinzelt zu finden ist. Drei Orten, in denen nur Präteritum gebraucht wird, stehen 125 Orte gegenüber, in denen nur Perfekt verwendet wird. In 24 Orten kommen beide Tempora vor. In einem dieser Orte überwiegt der Anteil an Präterita, in 17 der Anteil der Perfekte und in sechs Orten sind es von beiden Tempora gleich viel. Bei den drei Orten, in denen ausschließlich das Präteritum von wollen gebraucht wird, handelt es sich um Altheim im Landkreis Biberach (zwei Präteritumbildungen von einem Sprecher), Böhringen (zwei Präteritum-Belege von zwei Sprechern) und Furtwangen im Schwarzwald (ein Präteritum von einem Sprecher). Diese Auffälligkeiten liegen also vermutlich in der geringen Belegzahl begründet. Im Mittel- und Hochalemannischen sind in sieben Orten beide Tempora zu finden; im Niederalemannischen ist gar kein Präteritum nachweisbar. Im SSA gibt es also vereinzelt Präteritum-Belege. Das Perfekt macht allerdings die deutliche Mehrheit aus. Es gibt also 
keine auffälligen Unterschiede im Präteritumgebrauch innerhalb des Alemannischen Deutschlands.

In 42 der 46 Untersuchungsorte des Korpus REDI+FLARS (dt.) ist das Modalverb wollen in einem Tempus der einfachen Vergangenheit belegt. 371 Belege insgesamt entsprechen durchschnittlich 8,8 Belegen pro Ort. Mit einem Ort, in dem nur Perfekt zu finden ist, gegenüber zwölf, in denen nur Präteritum nachgewiesen wurde, ist der Anteil der Präteritumbildungen dabei deutlich höher als der Anteil der Perfektbildungen. In 29 Orten sind beide Tempora belegt: In 24 überwiegt der Anteil der Präterita, in fünf der Anteil der Perfekte. In Freiburg kommt bei sechs Sprechern, die sieben Präteritum- und zwei Perfektformen bilden, häufiger das Präteritum als das Perfekt vor. Allerdings ist der Präteritum-Anteil nicht höher als im restlichen Untersuchungsgebiet.

Es lässt sich also ein deutlicher Anstieg der Orte, in denen Präteritum gebraucht wird, und der Orte, in denen ausschließlich Präteritum verwendet wird, erkennen. Während im älteren Korpus noch das Perfekt deutlich überwiegt, macht nun das Präteritum den Großteil der Belege aus.

\section{Kopulaverb: sein}

Die Karten 32 und 33 illustrieren die areale Verteilung des Verbs sein im älteren (1974-1985) und im jüngeren (2007-2013) Untersuchungszeitraum.
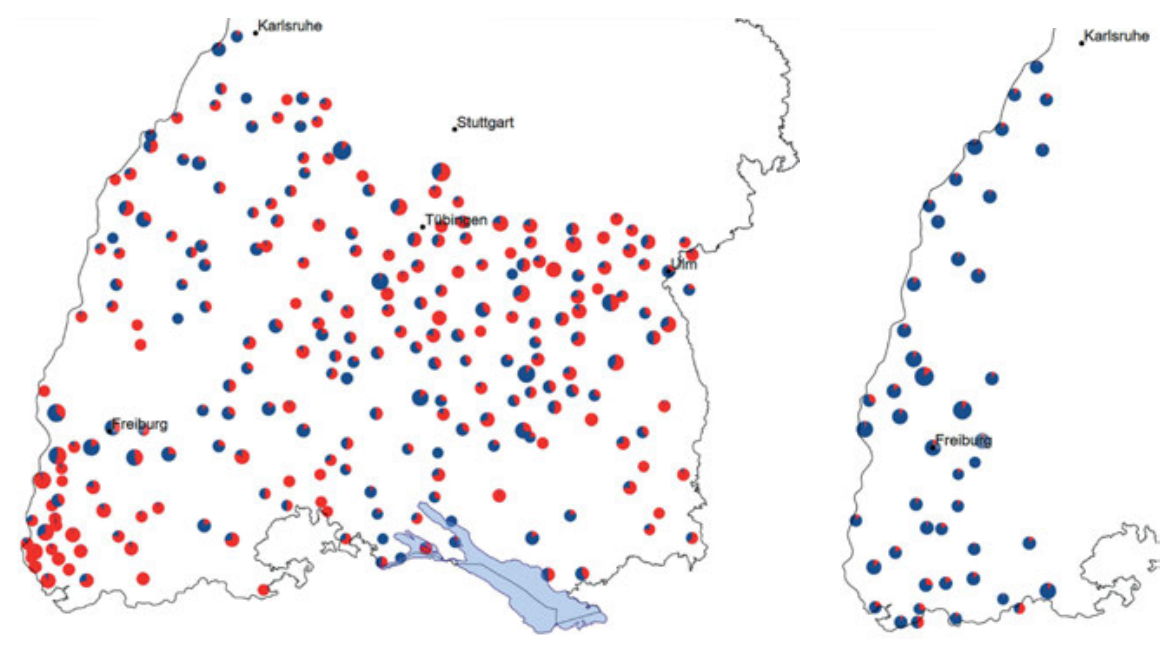

Karte 32 und 33: Areale Verteilung des Verbs sein in einfachen Vergangenheitstempora im SSA [links] und in REDI+FLARS (dt.) [rechts] 
Die beiden Tempusformen von sein kommen im Korpus SSA in 239 von 260 Orten vor. Bei 9156 Belegen insgesamt sind es durchschnittlich 38,3 Belege pro Ortspunkt. Bei der arealen Verteilung macht das Perfekt den Großteil der Belege aus. In 25 Erhebungsorten sind ausschließlich Perfektformen zu finden, in neun nur Präteritumformen und in 205 Orten beide Tempusformen. Durch die sehr hohe Belegzahl gibt es nur wenige Orte mit einem ausgeglichenen Perfekt-PräteritumVerhältnis. Dementsprechend kommen die beiden Tempora nur in acht Orten gleich häufig vor, in 73 überwiegt das Präteritum und in 124 das Perfekt. Besonders im Südwesten des Untersuchungsgebietes finden sich viele Orte, in denen nur Perfekt nachgewiesen werden kann. Im badischalemannischen Teilgebiet des SSA wird die Präteritumform von sein also etwas seltener verwendet als im restlichen Untersuchungsgebiet.

In REDI+FLARS (dt.) ist der Anteil an Präteritumbildungen deutlich höher als der Anteil der Perfektbildungen. In allen 46 Untersuchungsorten ist mindestens eine der beiden Formen zu finden. Auf diese verteilen sich 6867 Belege, d. h. im Durchschnitt 149,3 Belege pro Ort. Lediglich in einem Ort kommt nur Präteritum, in keinem nur Perfekt vor. In 45 Orten sind beide Tempora zu finden, wobei lediglich in einem Ort das Perfekt (Leibstadt $(\mathrm{CH})$ ) und in 44 das Präteritum (zum Teil sehr deutlich) überwiegt. Im Norden des Untersuchungsgebiets tritt das Präteritum häufiger auf als im Süden. Auffallend ist, dass in fast keinem Ort nur eines der beiden Tempora gebraucht wird. Ob dies auf die sehr hohe Belegzahl zurückzuführen ist oder ob es einen Bedeutungsunterschied zwischen den beiden Tempora gibt, wird in Kapitel 3.2 näher behandelt.

Der Vergleich der arealen Ausbreitung zwischen dem ersten und dem zweiten Untersuchungszeitraum zeigt eine deutliche Zunahme der Orte, in denen Präteritalformen zu finden sind, und eine deutliche Zunahme der Orte, in denen die Präteritalformen überwiegen. Während im SSA ein ausgewogenes Verhältnis von Perfekt und Präteritum herrscht, ist der Anteil an Präteritumformen gegenüber den Perfektformen in REDI+FLARS (dt.) deutlich höher. Allerdings ist das Perfekt bis auf einen Ortspunkt nirgendwo vollständig zurückgedrängt worden.

\section{Zusammenfassung}

Bei den Verben machen, sagen und mögen konnte (mit zwei Ausnahmen beim Verb sagen) weder im SSA noch in REDI+FLARS (dt.) eine Präteritalform nachgewiesen werden. Bei allen anderen Verben hat die areale Ausbreitung des Präteritums deutlich zugenommen. Dabei gilt: Je häufiger die Präteritalform eines Verbs im SSA auftritt, desto häufiger ist sie in REDI+FLARS (dt.) belegt. Dies zeigt sich in einer deutlich höheren Zahl an Ortspunkten, in denen Präteritum zu finden ist, und einer häufig höheren Zahl an Orten, in denen Präteritum die Mehrzahl der 
Belege ausmacht. Bei den Verben geben, kommen, werden, dürfen, können und müssen sind im SSA in fast allen Orten nur Perfektformen zu finden; in REDI+FLARS (dt.) ist das Perfekt-Präteritum-Verhältnis (beinahe) ausgeglichen. Bei den Verben haben und gehen kommt das Präteritum nur vereinzelt im Untersuchungsgebiet des SSA vor. In REDI+FLARS (dt.) ist der Anteil an Perfekt zwar immer noch höher; aber der des Präteritums hat deutlich zugenommen. Die Verben sollen und sein weisen bereits im SSA ein in etwa ausgeglichenes Verhältnis der beiden Tempora auf. In REDI+FLARS (dt.) macht bei diesen Verben nun das Präteritum die überwiegende Mehrheit an Belegen aus. Besonders auffällig ist die Verteilung beim Modalverb wollen. Bei diesem ist im SSA der Anteil an Perfekt deutlich größer als der des Präteritums; in REDI+FLARS (dt.) dagegen überwiegt das Präteritum deutlich.

Die folgende Tabelle 5 verdeutlicht die Anzahl an Ortspunkten, in denen Präteritum-Belege nachgewiesen werden konnten, im Vergleich mit der Anzahl an Orten, in denen nur Perfektformen belegt sind, im SSA und in REDI+FLARS (dt.).

Tab. 5: Absolute und relative Anzahl an Präteritum-Belegen in Ortspunkten

\begin{tabular}{|c|c|c|}
\hline & SSA & REDI+FLARS (dt.) \\
\hline machen & $0 / 213(0,0 \%)$ & $0 / 46 \quad(0,0 \%)$ \\
\hline sagen & $0 / 229(0,0 \%)$ & $2 / 46(4,3 \%)$ \\
\hline haben & $17 / 223(7,6 \%)$ & $43 / 46(93,5 \%)$ \\
\hline geben & $17 / 186(9,1 \%)$ & $38 / 44 \quad(86,4 \%)$ \\
\hline gehen & $16 / 203(7,9 \%)$ & $36 / 45 \quad(80,0 \%)$ \\
\hline kommen & $23 / 211 \quad(10,9 \%)$ & $42 / 45(93,3 \%)$ \\
\hline werden & $17 / 157 \quad(10,8 \%)$ & $36 / 44 \quad(81,8 \%)$ \\
\hline dürfen & $3 / 91 \quad(3,3 \%)$ & $15 / 24(62,5 \%)$ \\
\hline können & $14 / 182(7,7 \%)$ & $39 / 45 \quad(86,7 \%)$ \\
\hline mögen & $0 / 20 \quad(0,0 \%)$ & $0 / 4 \quad(0,0 \%)$ \\
\hline müssen & $15 / 199(7,5 \%)$ & $39 / 45 \quad(86,7 \%)$ \\
\hline sollen & $22 / 48 \quad(45,8 \%)$ & $18 / 21 \quad(85,7 \%)$ \\
\hline wollen & $27 / 152(17,8 \%)$ & $41 / 42(97,6 \%)$ \\
\hline sein & $214 / 239(89,5 \%)$ & $46 / 46(100 \%)$ \\
\hline
\end{tabular}

Zudem zeigt sich, dass es im SSA zwischen dem badischalemannischen Teilgebiet und dem gesamten Untersuchungsgebiet kaum Unterschiede im Perfekt- und Präteritumgebrauch gibt. Lediglich bei den Verben kommen und sein sind die 
Präteritumformen im badischalemannischen etwas seltener als im restlichen Untersuchungsgebiet. Angesichts dieser geringfügigen Unterschiede innerhalb des Alemannischen Deutschlands, das im SSA untersucht wurde, ist ein Vergleich der Korpora SSA und REDI+FLARS (dt.) möglich.

\subsubsection{Präteritum-Vorkommen und Größe der Erhebungsorte}

In Kapitel 3.1.1 wurde die Frage gestellt, ob die Präteritumformen nur in Städten vorzufinden sind (vgl. Rowley 1983: 165). Ich zeige daher in den folgenden Tabellen 6 und 7 das Vorkommen von Präteritum und Perfekt in den Erhebungsorten des Korpus REDI+FLARS (dt.) nach deren Größe unterschieden.

Für die Ermittlung der Einwohnerzahlen der einzelnen Orte wurde der Zensus von $2011^{15}$ herangezogen. Die Aufnahmen sind zwar von 2007 bis 2013 entstanden und damit teilweise bis zu vier Jahre vor beziehungsweise zwei Jahre nach der Erfassung durch den Zensus, allerdings erschien es gerade aufgrund der oft widersprüchlichen Angaben zur Einwohnerzahl wichtig, eine möglichst objektive Quelle heranzuziehen. In einigen Fällen konnte leider nicht auf den Zensus zugegriffen werden, da dieser einzelne Orte nicht erfasst hat, die entweder in der Schweiz liegen (Leibstadt und Rheinfelden) oder Ortsteile sind (Karsau, Neuenweg, Plittersdorf, Reichenbach und Todtnauberg). Bei diesen Erhebungsorten wurde auf die Schätzungen der jeweiligen Orte zurückgegriffen. In der Tabelle sind sie markiert $\left(^{\star}\right)$. Ein , $+^{\star}$ zeigt an, dass ein Präteritum (PR) beziehungsweise Perfekt (PF) des jeweiligen Verbs im Ort belegt ist, ein ,-` dagegen, dass keine Form im Korpus gebraucht wurde.

Tab. 6: Vorkommen von Präteritum und Perfekt einzelner irregulärer, starker und schwacher Verben in REDI+FLARS (dt.)

\begin{tabular}{lllllllllll}
\hline $\begin{array}{l}\text { Erhebungs- } \\
\text { ort }\end{array}$ & $\begin{array}{l}\text { Einwoh- } \\
\text { ner }\end{array}$ & sein & haben & $\begin{array}{l}\text { kom- } \\
\text { men }\end{array}$ & & geben & $\begin{array}{l}\text { wer- } \\
\text { den }\end{array}$ & gehen & sagen & machen \\
\cline { 3 - 9 } & & & $\mathrm{PR} / \mathrm{PF}$ & $\mathrm{PR} / \mathrm{PF}$ & $\mathrm{PR} / \mathrm{PF}$ & $\mathrm{PR} / \mathrm{PF}$ & $\mathrm{PR} / \mathrm{PF}$ & $\mathrm{PR} / \mathrm{PF}$ & $\mathrm{PR} / \mathrm{PF}$ & $\mathrm{PR} / \mathrm{PF}$ \\
Freiburg & 209.628 & $+/+$ & $+/+$ & $+/+$ & $+/+$ & $+/+$ & $+/+$ & $-/+$ & $-/+$ \\
Offenburg & 57.228 & $+/+$ & $+/+$ & $+/+$ & $+/+$ & $+/+$ & $+/+$ & $-/+$ & $-/+$ \\
Weil & 28.828 & $+/+$ & $+/+$ & $+/+$ & $+/+$ & $+/+$ & $+/+$ & $-/+$ & $-/+$ \\
\hline
\end{tabular}

15 Vgl. https://www.zensus2011.de/DE/Home/home_node.html 


\begin{tabular}{|c|c|c|c|c|c|c|c|c|c|}
\hline Achern & 24.523 & $+/+$ & $+/+$ & $+/+$ & $+/+$ & $-1+$ & $+/+$ & $-1+$ & $-1+$ \\
\hline Waldkirch & 20.789 & $+/+$ & $+/+$ & $+/+$ & $+/+$ & $+/+$ & $+/+$ & $-1+$ & $-1+$ \\
\hline Schopfheim & 18.582 & $+/+$ & $+/+$ & $+/+$ & $+/+$ & $+/+$ & $+/+$ & $-1+$ & $-1+$ \\
\hline $\begin{array}{l}\text { Bad Säckin- } \\
\text { gen }\end{array}$ & 16.208 & $+/+$ & $+/+$ & $+/+$ & $+1-$ & $+/+$ & $+/+$ & $-1+$ & $-1+$ \\
\hline Breisach & 13.992 & $+/+$ & $+/+$ & $+/+$ & $+/+$ & $+/+$ & $+/+$ & $-1+$ & $-1+$ \\
\hline $\begin{array}{l}\text { Rheinfelden } \\
\text { (CH) }\end{array}$ & $11.960^{*}$ & $+/+$ & $+/+$ & $+/+$ & $+1-$ & $+1-$ & $-1+$ & $-1+$ & $-1+$ \\
\hline Neuenburg & 11.626 & $+/+$ & $+/+$ & $-1+$ & $+/+$ & $+/-$ & $-1+$ & $-1+$ & $-1+$ \\
\hline Herbolzheim & 10.009 & $+/+$ & $+/+$ & $+/+$ & $+/+$ & $+/+$ & $+/+$ & $-1+$ & $-1+$ \\
\hline Endingen & 9.084 & $+/+$ & $+/+$ & $+/+$ & $+/+$ & $+/+$ & $-1+$ & $-1+$ & $-1+$ \\
\hline Tiengen & 8.110 & $+/+$ & $+/+$ & $+/+$ & $+/+$ & $+/+$ & $+/+$ & $-1+$ & $-1+$ \\
\hline Staufen & 7.349 & $+/+$ & $+/+$ & $+/+$ & $+/+$ & $+/+$ & $+/+$ & $-1+$ & $-1+$ \\
\hline Elzach & 7.091 & $+/+$ & $+/+$ & $+/+$ & $+/+$ & $+/+$ & $+/+$ & $-1+$ & $-1+$ \\
\hline Lichtental & $6.969^{\star}$ & $+/+$ & $+/+$ & $+/+$ & $+/-$ & $+/+$ & $+/+$ & $-1+$ & $-1+$ \\
\hline $\begin{array}{l}\text { Muggen- } \\
\text { sturm }\end{array}$ & 6.170 & $+/+$ & $+/+$ & $+/+$ & $+/+$ & $+/+$ & $+/+$ & $-1+$ & $-1+$ \\
\hline Bötzingen & 5.329 & $+/+$ & $+/+$ & $+/+$ & $+/+$ & $+/+$ & $+/+$ & $-1+$ & $-1+$ \\
\hline Münstertal & 5.044 & $+/+$ & $+/+$ & $+/+$ & $+/-$ & $-1+$ & $+/+$ & $-1+$ & $-1+$ \\
\hline Hügelsheim & 4.905 & $+/+$ & $+/+$ & $+/+$ & $+/-$ & $+/+$ & $+/+$ & $-1+$ & $-1+$ \\
\hline $\begin{array}{l}\text { Kappel-Gra- } \\
\text { fenhausen }\end{array}$ & 4.762 & $+/+$ & $-1+$ & $-1+$ & $-1+$ & $+1-$ & $-1+$ & $-1+$ & $-1+$ \\
\hline Herten & 4.500 & $+/+$ & $+/+$ & $+/+$ & $+/+$ & $+/+$ & $+/+$ & $+/+$ & $-1+$ \\
\hline Freistett & 3.970 & $+/+$ & $+/+$ & $+/+$ & $+/+$ & $+/+$ & $+/+$ & $-1+$ & $-1+$ \\
\hline Meißenheim & 3.748 & $+/+$ & $+/+$ & $+/+$ & $+/+$ & $+/+$ & $+/+$ & $-1+$ & $-1+$ \\
\hline Karsau & $3.670^{*}$ & $+/+$ & $+/+$ & $+/+$ & $+/+$ & $+/+$ & $-1+$ & $-1+$ & $-/+$ \\
\hline Au am Rhein & 3.287 & $+/+$ & $+/+$ & $+/+$ & $+/+$ & $+/+$ & $+/+$ & $-1+$ & $-1+$ \\
\hline Buchenbach & 3.168 & $+1-$ & $+/+$ & $+/+$ & $+/+$ & $-1+$ & $-1+$ & $-1+$ & $-1+$ \\
\hline Plittersdorf & $3.080^{*}$ & $+/+$ & $+/+$ & $+/+$ & $+/+$ & $-1+$ & $+/+$ & $-1+$ & $-1+$ \\
\hline Kork & 2.960 & $+/+$ & $+/+$ & $+/+$ & $+/+$ & $+/+$ & $+/+$ & $-1+$ & $-1+$ \\
\hline Oberried & 2.870 & $+/+$ & $+/+$ & $-1+$ & $+1-$ & $+/+$ & $+/+$ & $+/+$ & $-1+$ \\
\hline Herrischried & 2.663 & $+/+$ & $+/+$ & $+/+$ & $+/+$ & $+/+$ & $+/+$ & $-1+$ & $-1+$ \\
\hline St. Peter & 2.550 & $+/+$ & $+/+$ & $+/+$ & $+/+$ & $+/+$ & $+/+$ & $-/+$ & $-1+$ \\
\hline Auenheim & 2.420 & $+/+$ & $+/+$ & $+/+$ & $+/+$ & $-1+$ & $+/+$ & $-1+$ & $-/+$ \\
\hline $\begin{array}{l}\text { Schönen- } \\
\text { berg }\end{array}$ & 2.010 & $+/+$ & $+/+$ & $+/+$ & $-1+$ & $-1+$ & $-1+$ & $-1+$ & $-1+$ \\
\hline Greffern & 1.950 & $+/+$ & $+/+$ & $+/+$ & $+/+$ & $+/+$ & $+/+$ & $-1+$ & $-1+$ \\
\hline Todtmoos & 1.896 & $+/+$ & $+/+$ & $+/+$ & $+/+$ & $+/-$ & $+/+$ & $-1+$ & $-1+$ \\
\hline
\end{tabular}




\begin{tabular}{llllllllll}
\hline Malsburg & 1.485 & $+/+$ & $+/+$ & $+/+$ & $-/+$ & $-/+$ & $+/+$ & $-/+$ & $-/+$ \\
Leibstadt & $1.307^{*}$ & $+/+$ & $-/+$ & $-/-$ & $+/+$ & $-/-$ & $-/-$ & $-/+$ & $-/+$ \\
(CH) & & & & & & & & & \\
Hasel & 1.116 & $+/+$ & $+/+$ & $+/+$ & $+/+$ & $+/+$ & $+/+$ & $-/+$ & $-/+$ \\
Jechtingen & 1.110 & $+/+$ & $-/+$ & $-/+$ & $-/+$ & $-/+$ & $-/+$ & $-/+$ & $-/+$ \\
Buch & 810 & $+/+$ & $+/+$ & $-/-$ & $-/-$ & $+/+$ & $-/-$ & $-/+$ & $-/+$ \\
Todtnauberg & $740^{\star}$ & $+/+$ & $+/+$ & $+/+$ & $+/+$ & $+/+$ & $-/+$ & $-/+$ & $-/+$ \\
Bombach & 660 & $+/+$ & $+/+$ & $+/+$ & $+/+$ & $+/+$ & $+/+$ & $-/+$ & $-/+$ \\
Holzen & 570 & $+/+$ & $+/+$ & $+/+$ & $+/+$ & $+/+$ & $+/+$ & $-/+$ & $-/+$ \\
Reichen- & $400^{*}$ & $+/+$ & $+/+$ & $+/+$ & $+/+$ & $+/+$ & $+/+$ & $-/+$ & $-/+$ \\
bach & & & & & & & & & \\
Neuenweg & $330^{*}$ & $+/+$ & $+/+$ & $+/+$ & $-/+$ & $+/+$ & $+/+$ & $-/+$ & $-/+$ \\
\hline
\end{tabular}

Tab. 7: Vorkommen von Präteritum und Perfekt der Modalverben in REDI+FLARS (dt.)

\begin{tabular}{|c|c|c|c|c|c|c|c|}
\hline \multirow[t]{2}{*}{ Erhebungsort } & Einwohner & wollen & können & müssen & sollen & dürfen & mögen \\
\hline & & $\mathrm{PR} / \mathrm{PF}$ & $\mathrm{PR} / \mathrm{PF}$ & $\mathrm{PR} / \mathrm{PF}$ & $\mathrm{PR} / \mathrm{PF}$ & $\mathrm{PR} / \mathrm{PF}$ & $\mathrm{PR} / \mathrm{PF}$ \\
\hline Freiburg & 209.628 & $+/+$ & $+/+$ & $+/+$ & $+1-$ & $+1-$ & $-1-$ \\
\hline Offenburg & 57.228 & $+/+$ & $+/+$ & $+/+$ & $+/-$ & $-1+$ & $-1-$ \\
\hline Weil & 28.828 & $+/+$ & $+/+$ & $+/+$ & $-1-$ & $-1-$ & $-1-$ \\
\hline Achern & 24.523 & $+1+$ & $+/+$ & $+/+$ & $-1-$ & $-1-$ & $-1-$ \\
\hline Waldkirch & 20.789 & $+/+$ & $+/+$ & $+/+$ & $+1-$ & $+/+$ & $-1-$ \\
\hline Schopfheim & 18.582 & $+/+$ & $-1+$ & $-1+$ & $-1-$ & $-1-$ & $-1-$ \\
\hline Bad Säckingen & 16.208 & $-1-$ & $+1-$ & $+/+$ & $-1-$ & $-1-$ & $-1-$ \\
\hline Breisach & 13.992 & $+/+$ & $+1+$ & $+/+$ & $+1-$ & $+/+$ & $-1-$ \\
\hline $\begin{array}{l}\text { Rheinfelden } \\
\text { (CH) }\end{array}$ & $11.960 *$ & $+/+$ & $+/+$ & $+/+$ & $-1-$ & $-1+$ & $-1-$ \\
\hline Neuenburg & 11.626 & $+1-$ & $+/+$ & $+/+$ & $-1-$ & $+1-$ & $-1-$ \\
\hline Herbolzheim & 10.009 & $+/+$ & $+/+$ & $+/+$ & $+1-$ & $+1-$ & $-1-$ \\
\hline Endingen & 9.084 & $+/+$ & $+/+$ & $+/+$ & $-1+$ & $-1-$ & $-1-$ \\
\hline Tiengen & 8.110 & $+/+$ & $+/+$ & $+/+$ & $-1-$ & $+/+$ & $-1-$ \\
\hline Staufen & 7.349 & $+1-$ & $+/+$ & $+/+$ & $-1-$ & $+/-$ & $-1-$ \\
\hline Elzach & 7.091 & $+/+$ & $+/+$ & $+/+$ & $-1-$ & $+1-$ & $-1-$ \\
\hline Lichtental & $6.969^{\star}$ & $-1-$ & $+/+$ & $+/+$ & $+/-$ & $+1-$ & $-1-$ \\
\hline Muggensturm & 6.170 & $+1-$ & $+/+$ & $+/+$ & $+1-$ & $-1-$ & $-1-$ \\
\hline Bötzingen & 5.329 & $+/+$ & $+/+$ & $+/+$ & $+1-$ & $+1-$ & $-1-$ \\
\hline Münstertal & 5.044 & $+/+$ & $+/+$ & $+/+$ & $+1-$ & $-1-$ & $-1-$ \\
\hline
\end{tabular}




\begin{tabular}{|c|c|c|c|c|c|c|c|}
\hline Hügelsheim & 4.905 & $+1-$ & $+/+$ & $-/+$ & $+/-$ & $+1-$ & $-1-$ \\
\hline $\begin{array}{l}\text { Kappel-Grafen- } \\
\text { hausen }\end{array}$ & 4.762 & $+/+$ & $-1+$ & $+/+$ & $+/-$ & $-1+$ & $-1-$ \\
\hline Herten & 4.500 & $+1-$ & $+/+$ & $+/+$ & $+1-$ & $-1-$ & $-1-$ \\
\hline Freistett & 3.970 & $+/+$ & $+/+$ & $+/+$ & $-1-$ & $+/-$ & $-1-$ \\
\hline Meißenheim & 3.748 & $+/+$ & $+/+$ & $+1-$ & $-1-$ & $-1+$ & $-1-$ \\
\hline Karsau & $3.670^{\star}$ & $+1-$ & $+1-$ & $+/+$ & $-1-$ & $-1-$ & $-1-$ \\
\hline Au am Rhein & 3.287 & $+1-$ & $+/+$ & $+/+$ & $-1-$ & $-1-$ & $-1-$ \\
\hline Buchenbach & 3.168 & $+/-$ & $-1-$ & $-1+$ & $+1-$ & $-1-$ & $-1-$ \\
\hline Plittersdorf & $3.080^{\star}$ & $+/+$ & $-1+$ & $+/+$ & $+1-$ & $-1+$ & $-/+$ \\
\hline Kork & 2.960 & $+/+$ & $+/+$ & $-1+$ & $+1-$ & $-1+$ & $-1-$ \\
\hline Oberried & 2.870 & $+/-$ & $+/+$ & $+/-$ & $+1-$ & $-1-$ & $-1-$ \\
\hline Herrischried & 2.663 & $+/+$ & $-1+$ & $+/+$ & $-1+$ & $-1+$ & $-1-$ \\
\hline St. Peter & 2.550 & $+/+$ & $+/+$ & $+/+$ & $+1-$ & $-1+$ & $-/+$ \\
\hline Auenheim & 2.420 & $+/+$ & $+/+$ & $-1-$ & $-1-$ & $+/+$ & $-1-$ \\
\hline Schönenberg & 2.010 & $-1-$ & $+/+$ & $-/+$ & $+/+$ & $-1-$ & $-1-$ \\
\hline Greffern & 1.950 & $+/+$ & $+/+$ & $+/+$ & $+1-$ & $+/+$ & $-1-$ \\
\hline Todtmoos & 1.896 & $+/-$ & $+/+$ & $+/-$ & $+/-$ & $-1-$ & $-1-$ \\
\hline Malsburg & 1.485 & $-1+$ & $+/+$ & $+/+$ & $+1-$ & $-1-$ & $-1-$ \\
\hline Leibstadt (CH) & $1.307^{\star}$ & $+1-$ & $+/+$ & $+/-$ & $+/-$ & $-1-$ & $-1-$ \\
\hline Hasel & 1.116 & $+/+$ & $+/+$ & $+/+$ & $+1-$ & $-1-$ & $-/+$ \\
\hline Jechtingen & 1.110 & $+/+$ & $-1+$ & $-1+$ & $-1-$ & $-1-$ & $-1-$ \\
\hline Buch & 810 & $-1-$ & $-1+$ & $-1-$ & $-1+$ & $-1-$ & $-1-$ \\
\hline Todtnauberg & $740^{\star}$ & $-1-$ & $+/+$ & $+/+$ & $+1-$ & $-1-$ & $-1-$ \\
\hline Bombach & 660 & $+/+$ & $+/+$ & $+/+$ & $+1-$ & $+/+$ & $-1+$ \\
\hline Holzen & 570 & $+/+$ & $+/+$ & $+/+$ & $+/-$ & $-1+$ & $-1-$ \\
\hline Reichenbach & $400^{*}$ & $+/+$ & $+/+$ & $+/+$ & $+/-$ & $-1-$ & $-1-$ \\
\hline Neuenweg & $330^{\star}$ & $+/-$ & $+/-$ & $+/+$ & $+/-$ & $-1-$ & $-1-$ \\
\hline
\end{tabular}

Mit Blick auf die Tabellen 6 und 7 kann die Frage nach der Begrenzung von Präteritalformen auf größere Orte klar verneint werden. Selbst in den kleinsten Orten Neuenweg mit 330 und Reichenbach mit 400 Einwohnern kommen von den 14 zur Untersuchung herangezogenen Verben neun bzw. zehn im Präteritum vor.

Es stellt sich allerdings die Frage, ob in größeren Orten mehr Verben im Präteritum vorkommen als in kleineren Orten. Um diese Frage zu beantworten, habe ich einen bivariaten Korrelationstest nach Pearson durchgeführt. Der damit ermittelte Korrelationskoeffizient $\mathrm{R}^{2}$ kann Werte zwischen -1 und +1 annehmen. Ein Wert von -1 zeigt einen perfekt negativen Zusammenhang an; ein Wert von +1 
einen perfekt positiven. Hat der Koeffizient $\mathrm{R}^{2}$ den Wert 0 besteht überhaupt kein linearer Zusammenhang zwischen den beiden Werten.

Der Pearson-Koeffizient ergibt allerdings hier ein nicht-signifikantes Ergebnis. Die nachfolgende Abbildung 4 verdeutlicht die nicht-signifikante Korrelation zwischen Einwohnerzahl und Anzahl der Verbtypes.

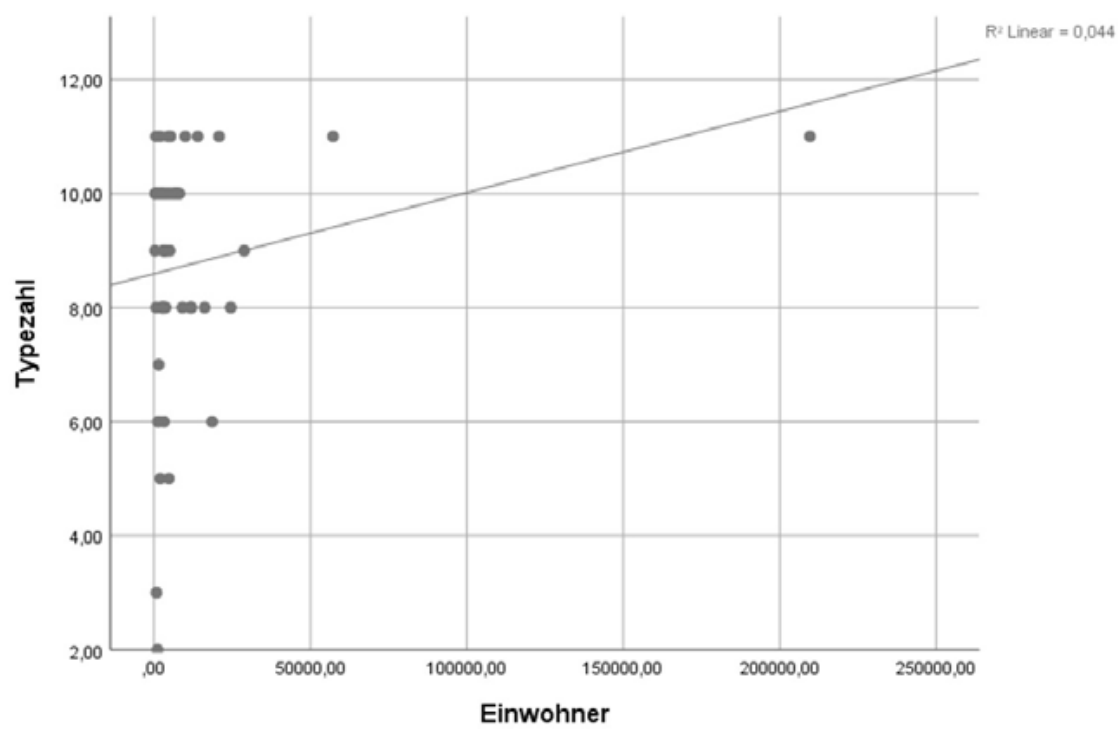

Abb. 4: Einfache Streuung mit Anpassungslinie für den Zusammenhang zwischen Verbtypezahl und Anzahl an Einwohnern

Das heißt, die Einwohnerzahl und die Anzahl der (hier untersuchten) Verbtypes korrelieren nicht signifikant miteinander. Obwohl einige Ausreißer diesen Eindruck erwecken mögen (vgl. Freiburg und Offenburg mit elf Verbtypes gegenüber Buch und Jechtingen mit drei bzw. zwei Verbtypes), ist das Präterituminventar in den großen Orten nicht größer als in den kleineren Orten.

Da in dieser Arbeit spontansprachliche Daten untersucht wurden, kann nicht garantiert werden, dass alle Verbtypes in jedem Ort vorkommen. Dementsprechend ist in vielen Orten für einige Verben weder eine Perfekt- noch eine Präteritumform belegt. Da diese Verben als 'Präteritum nicht vorhanden' gemessen werden, handelt es sich dabei um einen Störfaktor. Um diesen Störfaktor herauszurechnen, habe ich die Anzahl der Präteritumformen, die in einem Ort zu finden waren, durch die Anzahl an Verben geteilt, die entweder im Perfekt oder im Präteritum im jeweiligen Ort belegt sind und anschließend mit zehn 
multipliziert. Bei Achern beispielsweise kommen drei der 14 Verben (dürfen, mögen und sollen) weder im Perfekt noch im Präteritum vor. Die sieben nachweisbaren Präteritalformen in Achern werden also durch elf geteilt (ergibt 0,73 ) und mit zehn multipliziert (ergibt 7,3). Die untenstehende Abbildung 5 illustriert das Ergebnis.

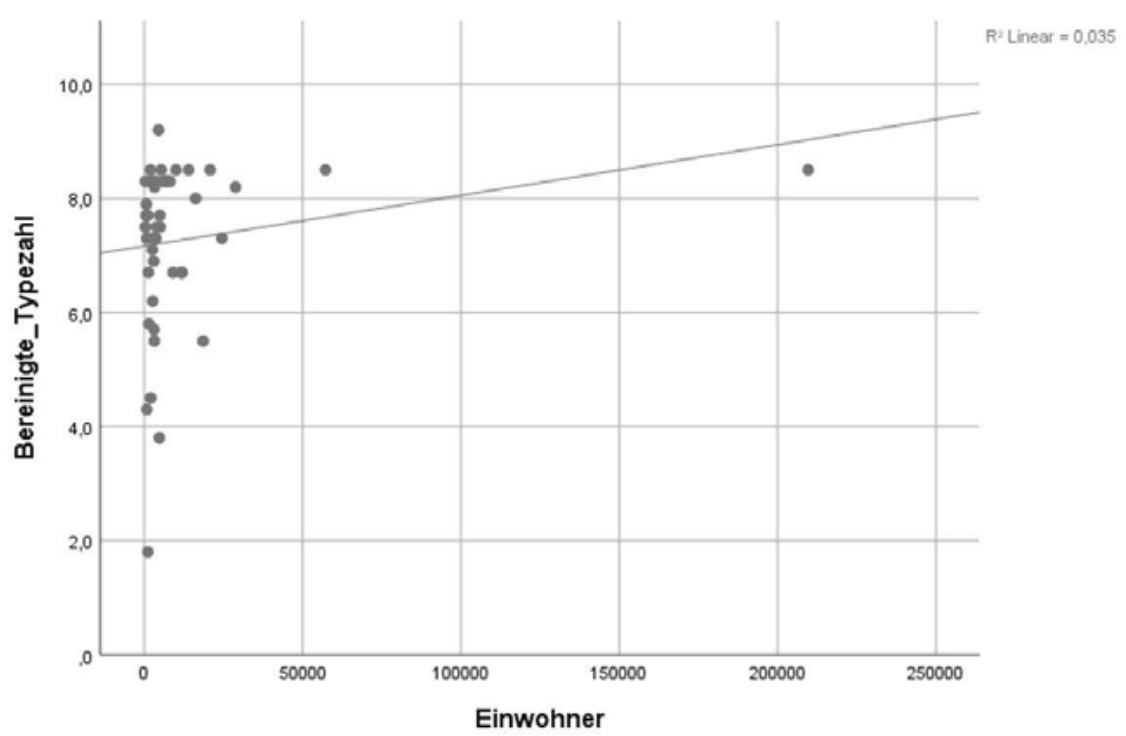

Abb. 5: Einfache Streuung mit Anpassungslinie für den Zusammenhang zwischen bereinigter Verbtypezahl und Anzahl an Einwohnern

Auch für die bereinigte Verbtypezahl ergibt der bivariate Korrelationstest nach Pearson allerdings kein signifikantes Ergebnis.

Um eine Vergleichbarkeit zwischen den Orten herzustellen, kann es auch sinnvoll sein, Orte auszuschließen, in denen mindestens ein Verb weder im Perfekt noch im Präteritum vorkommt. Da die Modalverben in sehr vielen Orten in keinem der beiden Tempora vorkommen, betrachte ich lediglich die Vollverben. Somit schließe ich die Orte Buch und Leibstadt (CH) für diese Korrelationsanalyse aus. Auch diese Analyse zeigt, dass es keinen Zusammenhang zwischen Einwohnerzahl und Anzahl an Verben gibt ( $R^{2}$ beträgt 0,017 ).

Ausgehend von den 14 Verben aus frequenten schwachen, starken und irregulären Verben sowie Modalverben lässt sich daher festhalten, dass das Formeninventar für das Präteritum nicht mit der Einwohnerzahl des Erhebungsortes korreliert. Das Präteritum ist vielmehr von Großstädten wie Freiburg bis hin zu 
kleinen Dörfern wie Neuenweg gleichermaßen vorhanden. Dieses Ergebnis deckt sich auch mit den oben dargestellten Befunden für die areale Ausbreitung des Präteritums. Im folgenden Kapitel 3.1.5 möchte ich nun der Frage nachgehen, wie die Frequenz der Präteritalformen vom ersten zum zweiten Untersuchungszeitraum zugenommen hat.

\subsubsection{Zunahme der Präteritalformen in der Zeit}

Dieses Kapitel betrachtet die Häufigkeit des Vorkommens der Verben in Perfekt und Präteritum. ${ }^{16}$ Dabei möchte ich in der real time und in der apparent time untersuchen, ob die Frequenz der Präteritalformen gegenüber den Perfektformen für die Verben zugenommen hat. Dazu werden zunächst die Korpora SSA und REDI+FLARS (dt.) und anschließend die ältere mit der jüngeren Sprechergruppe in REDI+FLARS (dt.) verglichen. Ich ziehe diejenigen Verben zum Vergleich heran, deren Präteritum in mindestens einem der beiden Korpora mindestens zweimal vorkommt. Das sind neben den bereits in Kapitel 3.1.3 vorgestellten Verben (sagen, haben, geben, gehen, kommen, werden, dürfen, können, müssen, sollen, wollen und sein) die Verben aussehen, denken, finden, halten, heißen, kennen, lassen, liegen, sitzen, stehen und wissen. Hierbei sind zum Teil verschiedene Verben zusammengefasst. Grundlegend war dafür, dass sie sich in der Grundbedeutung (und dementsprechend in der Aktionsart) nicht voneinander unterscheiden. So werden beispielsweise die Verben heimkommen und hinkommen, die im Duden als einzelne Verben gelistet sind (Duden 2017: 538 und 549), zum Verb kommen gezählt, da sie die Grundbedeutung von kommen lediglich spezifizieren, aber nicht grundlegend ändern. Im Gegensatz dazu bewerte ich das Verb aussehen als eigenes Verb, da es sich in semantischer und morpho-syntaktischer Valenz vom Verb sehen unterscheidet.

Tabelle 8 zeigt die quantitative Verteilung der Präterital- und Perfektformen bei den jeweiligen Verben im SSA; Tabelle 9 in REDI+FLARS (dt.).

16 Teile dieses Unterkapitels wurden mit einer Teilmenge der Daten bereits in Leonhard (2021) publiziert. 
Tab. 8: Absolute und relative Häufigkeiten von Perfekt und Präteritum einzelner Verben im Korpus SSA

\begin{tabular}{|c|c|c|c|}
\hline & Perfekt & Präteritum & Gesamt \\
\hline denken & 270 (100\%) & $0 \quad(0 \%)$ & $270(100 \%)$ \\
\hline halten & 119 (100\%) & $0 \quad(0 \%)$ & 119 (100\%) \\
\hline kennen & $186(100 \%)$ & $0 \quad(0 \%)$ & $186(100 \%)$ \\
\hline lassen & 132 (100\%) & $0 \quad(0 \%)$ & 132 (100\%) \\
\hline sagen & 3730 (100\%) & $0 \quad(0 \%)$ & 3730 (100\%) \\
\hline wissen & $229(99,6 \%)$ & $1(0,43 \%)$ & $230(100 \%)$ \\
\hline haben & 3920 (99,4\%) & $22(0,56 \%)$ & 3942 (100\%) \\
\hline müssen & $2103(99,1 \%)$ & $20(0,94 \%)$ & $2123(100 \%)$ \\
\hline sitzen & $75(98,7 \%)$ & $1(1,3 \%)$ & $76(100 \%)$ \\
\hline heißen & $482(98,4 \%)$ & $8(1,6 \%)$ & $490(100 \%)$ \\
\hline dürfen & $195(98,0 \%)$ & $4(2,0 \%)$ & 199 (100\%) \\
\hline können & 971 (98,0\%) & $20(2,0 \%)$ & 991 (100\%) \\
\hline finden & 44 (97,8\%) & 1 (2,2\%) & 45 (100\%) \\
\hline gehen & $1138(97,8 \%)$ & $26(2,2 \%)$ & 1164 (100\%) \\
\hline kommen & $1896(96,5 \%)$ & $69(3,5 \%)$ & $1965(100 \%)$ \\
\hline geben & 857 (96,3\%) & $33(3,7 \%)$ & $890(100 \%)$ \\
\hline aussehen & $40(95,2 \%)$ & $2(4,8 \%)$ & $42(100 \%)$ \\
\hline wollen & 499 (93,4\%) & $35(6,6 \%)$ & $534(100 \%)$ \\
\hline liegen & 101 (92,7\%) & $8(7,3 \%)$ & $109(100 \%)$ \\
\hline stehen & 292 (92,4\%) & $24(7,6 \%)$ & $316(100 \%)$ \\
\hline werden & $385(87,7 \%)$ & $54(12,3 \%)$ & 439 (100\%) \\
\hline sein & $5239(57,2 \%)$ & 3917 (42,8\%) & $9156(100 \%)$ \\
\hline sollen & $38(55,9 \%)$ & $30(44,1 \%)$ & 68 (100\%) \\
\hline
\end{tabular}

Im Korpus SSA ist bei den meisten Verben eine sehr geringe Präteritumfrequenz festzustellen. 17 der 23 Verben weisen in weniger als 5\% Präteritum auf. Bei fünf dieser Verben ist gar kein Präteritum belegt (denken, halten, kennen, lassen und sagen). Das Präteritum der Verben liegen (7,3\%), stehen (7,6\%), werden (12,3\%) und wollen (6,6\%) ist etwas häufiger. Auffällig ist, dass 47 der 54 Präteritalformen des Verbs werden Hilfsverben zur Passivkonstruktion sind. Nur sieben der 54 Belege sind Kopulaverben. Lediglich bei den zwei Verben sollen $(44,1 \%)$ und sein $(42,8 \%)$ ist das Präteritum in etwa so oft belegt wie das Perfekt. 
Tab. 9: Absolute und relative Häufigkeiten von Perfekt und Präteritum einzelner Verben im Korpus REDI+FLARS (dt.)

\begin{tabular}{|c|c|c|c|}
\hline & Perfekt & Präteritum & Gesamt \\
\hline sagen & 1997 (99,9\%) & $2(0,10 \%)$ & 1999 (100\%) \\
\hline halten & 35 (92,1\%) & $3(7,9 \%)$ & 38 (100\%) \\
\hline denken & $183(92,0 \%)$ & $16(8,0 \%)$ & 199 (100\%) \\
\hline sitzen & 111 (89,5\%) & $13(10,5 \%)$ & 124 (100\%) \\
\hline lassen & $26(86,7 \%)$ & $4(13,3 \%)$ & 30 (100\%) \\
\hline kennen & 69 (84,1\%) & $13(15,9 \%)$ & 82 (100\%) \\
\hline haben & $1233(77,4 \%)$ & $360(22,6 \%)$ & 1593 (100\%) \\
\hline wissen & $119(76,8 \%)$ & $36(23,2 \%)$ & 155 (100\%) \\
\hline kommen & 735 (75,6\%) & $237(24,4 \%)$ & 972 (100\%) \\
\hline stehen & $63(75,0 \%)$ & $21(25,0 \%)$ & 84 (100\%) \\
\hline gehen & $370 \quad(72,0 \%)$ & $144(28,0 \%)$ & 514 (100\%) \\
\hline liegen & 23 (71,9\%) & $9(28,1 \%)$ & 32 (100\%) \\
\hline heißen & $115(71,0 \%)$ & $47(29,0 \%)$ & $162(100 \%)$ \\
\hline aussehen & $7(63,6 \%)$ & $4(36,4 \%)$ & 11 (100\%) \\
\hline müssen & $298(62,3 \%)$ & $180(37,7 \%)$ & 478 (100\%) \\
\hline können & $208(53,1 \%)$ & $184(46,9 \%)$ & 392 (100\%) \\
\hline werden & $171(50,3 \%)$ & 169 (49,7\%) & 340 (100\%) \\
\hline finden & $42(47,7 \%)$ & $46(52,3 \%)$ & 88 (100\%) \\
\hline geben & $168(42,5 \%)$ & $227(57,5 \%)$ & $395(100 \%)$ \\
\hline dürfen & $28(41,8 \%)$ & $39(58,2 \%)$ & 67 (100\%) \\
\hline wollen & $94(25,3 \%)$ & $277(74,7 \%)$ & 371 (100\%) \\
\hline sollen & $5(12,2 \%)$ & $36(87,8 \%)$ & $41(100 \%)$ \\
\hline sein & 665 (9,7\%) & 6202 (90,3\%) & 6867 (100\%) \\
\hline
\end{tabular}

In REDI+FLARS (dt.) zeigt sich ein anderes Bild. Für jedes dieser Verben sind Präteritalformen belegt. Dabei hat die Frequenz des Präteritums bei jedem der Verben zugenommen. Das Verb sagen hat allerdings mit zwei Belegen $(0,10 \%)$ auch in REDI+FLARS (dt.) eine sehr geringe Belegfrequenz, sodass diese Präteritalverwendungen eher als Ausnahmen zu betrachten sind.

Die nachfolgende Tabelle 10 stellt die relative Präteritumfrequenz der einzelnen Verben für SSA und REDI+FLARS (dt.) gegenüber. 
Tab. 10: Relative Häufigkeiten des Präteritums einzelner Verben in den Korpora SSA und REDI+FLARS (dt.)

\begin{tabular}{|c|c|c|c|}
\hline & $\begin{array}{l}\text { Präteritum } \\
\text { SSA }\end{array}$ & $\begin{array}{l}\text { Präteritum REDI+FLARS } \\
\text { (dt.) }\end{array}$ & $\begin{array}{l}\text { Differenz der relativen } \\
\text { Präteritumfrequenz }\end{array}$ \\
\hline sagen & $0 \%$ & $0,10 \%$ & $0,10 \%$ \\
\hline halten & $0 \%$ & $7,9 \%$ & $7,9 \%$ \\
\hline denken & $0 \%$ & $8,0 \%$ & $8,0 \%$ \\
\hline sitzen & $1,3 \%$ & $10,5 \%$ & $9,2 \%$ \\
\hline lassen & $0 \%$ & $13,3 \%$ & $13,3 \%$ \\
\hline kennen & $0 \%$ & $15,9 \%$ & $15,9 \%$ \\
\hline stehen & $7,6 \%$ & $25,0 \%$ & $17,4 \%$ \\
\hline liegen & $7,3 \%$ & $28,1 \%$ & $20,8 \%$ \\
\hline kommen & $3,5 \%$ & $24,4 \%$ & $20,9 \%$ \\
\hline haben & $0,56 \%$ & $22,6 \%$ & $22,0 \%$ \\
\hline wissen & $0,43 \%$ & $23,2 \%$ & $22,8 \%$ \\
\hline gehen & $2,2 \%$ & $28,0 \%$ & $25,8 \%$ \\
\hline heißen & $1,6 \%$ & $29,0 \%$ & $27,4 \%$ \\
\hline aussehen & $4,8 \%$ & $36,4 \%$ & $31,6 \%$ \\
\hline müssen & $0,94 \%$ & $37,7 \%$ & $36,8 \%$ \\
\hline werden & $12,3 \%$ & $49,7 \%$ & $37,4 \%$ \\
\hline sollen & $44,1 \%$ & $87,8 \%$ & $43,7 \%$ \\
\hline können & $2,0 \%$ & $46,9 \%$ & $44,9 \%$ \\
\hline sein & $42,8 \%$ & $90,3 \%$ & $47,5 \%$ \\
\hline finden & $2,2 \%$ & $52,3 \%$ & $50,1 \%$ \\
\hline geben & $3,7 \%$ & $57,5 \%$ & $53,8 \%$ \\
\hline dürfen & $2,0 \%$ & $58,2 \%$ & $56,2 \%$ \\
\hline wollen & $6,6 \%$ & $74,7 \%$ & $68,1 \%$ \\
\hline
\end{tabular}

Die Präteritalformen der Verben denken (8,0\%), halten (7,9\%), kennen (15,9\%) und lassen (13,3\%), die im SSA gar kein Präteritum aufweisen, sind durchschnittlich seltener als die der restlichen Verben. Bei den zwölf Verben aussehen, finden, geben, gehen, haben, heißen, kommen, sitzen, wissen, dürfen, können und müssen sind im SSA Präteritalformen belegt, aber das Präteritum macht weniger als 5\% der Belege aus. In REDI+FLARS (dt.) weisen diese Verben Frequenzen zwischen 10,5\% beim Verb sitzen (1,3\% im SSA) und 58,2\% beim Verb dürfen (2,0\% im SSA) auf. Die Präteritalformen der Verben liegen (7,3\% im SSA), stehen (7,6\% im SSA), 
werden (12,3\% im SSA) und wollen (6,6\% im SSA) sind dagegen zum Korpus REDI+FLARS (dt.) mit 28,1\% (liegen), 25,0\% (stehen), 49,7\% (werden) und 74,7\% (wollen) mit Ausnahme von wollen verhältnismäßig schwach gewachsen. Auch hier fällt beim Verb werden der hohe Anteil an Hilfsverben zur Passivbildung auf. 156 der 169 Belege sind Auxiliare; 13 sind Kopulaverben. Die vergleichsweise geringe Zunahme könnte zumindest bei den Verben liegen und stehen mit der geringen Frequenz dieser Verben erklärt werden. Während für das Verb stehen nur 84 Belege in Perfekt oder Präteritum zu finden sind, sind es beim Verb liegen sogar nur 32 Belege. Bei den Verben sollen und sein, die bereits im SSA mit 44,1\% bzw. 42,8\% vergleichsweise häufig im Präteritum gebraucht werden, überwiegt in REDI+FLARS (dt.) nun das Präteritum mit 87,8\% bzw. 90,3\% deutlich.

Ein bivariater Korrelationstest nach Pearson, der die relative Häufigkeit des Präteritums der einzelnen Verben im SSA und in REDI+FLARS (dt.) vergleicht, ergibt ein höchstsignifikantes Ergebnis für den Zusammenhang zwischen relativer Häufigkeit des Präteritums im SSA und in REDI+FLARS (dt.). Für diesen Test wurde das Verb sagen ausgeschlossen, da dessen Präteritalform auch in REDI+FLARS (dt.) fast nicht vorkommt. Die Korrelation nach Pearson ist mit einem Wert von $0,747^{\star \star \star}(\mathrm{n}=22)$ stark. Der Korrelationskoeffizient $\mathrm{R}^{2}$ beträgt dementsprechend 0,558, d. h. 55,8\% der einen Variablen lassen sich durch die andere Variable vorhersehen.

Die nachfolgende Abbildung 6 verdeutlicht diese höchstsignifikante Korrelation. Auf der y-Achse liegen die prozentualen Anteile an Präteritalverwendungen gegenüber den Perfektverwendungen der jeweiligen Verben in REDI+FLARS (dt.), auf der x-Achse die Präteritalverwendungen im SSA. Es zeigt sich ein linear positiver Zusammenhang zwischen der relativen Präteritalverwendung in REDI+FLARS (dt.) und im SSA. 


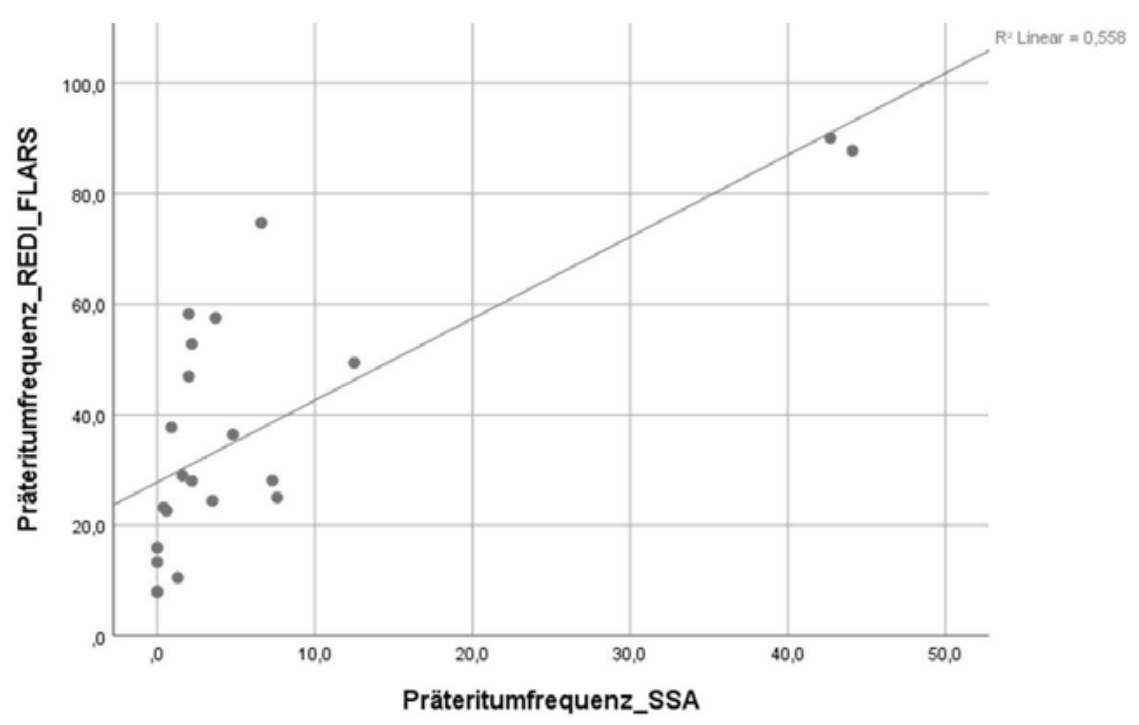

Abb. 6: Einfache Streuung mit Anpassungslinie für den Zusammenhang zwischen relativer Präteritumfrequenz in REDI+FLARS (dt.) und im SSA

Die Verben sein und sollen korrelieren dabei allerdings besonders stark. Schließt man diese beiden Verben aus der Korrelationsanalyse aus, ergibt sich kein signifikanter Zusammenhang zwischen der Frequenz der Verben im SSA und in REDI+FLARS (dt.).

In Leonhard (2021) wurden für den Vergleich von Perfekt und Präteritum lediglich die Untersuchungsorte betrachtet, die in beiden Korpora enthalten sind. Der Vergleich dieser Ergebnisse mit den hier präsentierten zeigt, dass es hinsichtlich des Tempusgebrauchs kaum Unterschiede zum Gesamtgebiet gibt (vgl. Leonhard 2021: 7-11). Diese Tatsache unterstreicht die Möglichkeit eines stringenten Vergleichs der Korpora SSA und REDI+FLARS (dt.).

Der Apparent-Time-Vergleich der älteren mit der jüngeren Sprechergruppe in REDI+FLARS (dt.) zeigt, dass die jüngere Sprechergruppe die meisten Verben noch häufiger im Präteritum verwendet als die ältere Gruppe. Tabelle 11 verdeutlicht das. Berücksichtigt wurden in dieser Tabelle nur Verben, bei denen mindestens zehn Präteritum-Belege in REDI+FLARS (dt.) vorkommen. Da bei der Erhebung das Alter einiger Sprecher nicht festgehalten wurde und diese Sprecher daher keiner der beiden Gruppen zugeordnet werden konnten, weicht die Zahl der Präteritum-Belege einiger Verben geringfügig von der Anzahl im Gesamtkorpus REDI+FLARS (dt.) ab. 
Tab. 11: Absolute und relative Häufigkeiten des Präteritums einzelner Verben in der älteren und der jüngeren Sprechergruppe in REDI+FLARS (dt.)

\begin{tabular}{|c|c|c|c|c|c|}
\hline \multirow[b]{2}{*}{ sollen } & \multicolumn{2}{|c|}{$\begin{array}{l}\text { Präteritum REDI+FLARS (dt.) } \\
\text { (alt) }\end{array}$} & \multicolumn{2}{|c|}{$\begin{array}{l}\text { Präteritum REDI+FLARS (dt.) } \\
\text { (jung) }\end{array}$} & \multirow{2}{*}{$\begin{array}{l}\text { Differenz der rela- } \\
\text { tiven Präteritum- } \\
\text { frequenz } \\
-4,4 \%\end{array}$} \\
\hline & $28 / 30$ & $(93,3 \%)$ & $8 / 9$ & $(88,9 \%)$ & \\
\hline stehen & $16 / 63$ & $(25,4 \%)$ & $3 / 13$ & $(23,1 \%)$ & $-2,3 \%$ \\
\hline sitzen & $8 / 68$ & $(11,8 \%)$ & $5 / 50$ & $(10,0 \%)$ & $-1,8 \%$ \\
\hline sein & $4299 / 4868$ & $(88,3 \%)$ & $1696 / 1769$ & $(95,9 \%)$ & $+7,6 \%$ \\
\hline denken & $3 / 119$ & $(2,5 \%)$ & $12 / 68$ & $(17,6 \%)$ & $+15,1 \%$ \\
\hline gehen & $93 / 374$ & $(24,9 \%)$ & $48 / 117$ & $(41,0 \%)$ & $+16,1 \%$ \\
\hline wollen & $198 / 277$ & $(71,5 \%)$ & $73 / 83$ & $(88,0 \%)$ & $+16,5 \%$ \\
\hline kommen & $152 / 723$ & $(21,0 \%)$ & $78 / 205$ & $(38,0 \%)$ & $+17,0 \%$ \\
\hline geben & $148 / 278$ & $(53,2 \%)$ & $69 / 97$ & $(71,1 \%)$ & $+17,9 \%$ \\
\hline werden & $105 / 238$ & $(44,1 \%)$ & $64 / 95$ & $(67,4 \%)$ & $+23,3 \%$ \\
\hline haben & $195 / 1120$ & $(17,4 \%)$ & $158 / 385$ & $(41,0 \%)$ & $+23,6 \%$ \\
\hline dürfen & $24 / 47$ & $(51,1 \%)$ & $13 / 17$ & $(76,5 \%)$ & $+25,4 \%$ \\
\hline können & $116 / 289$ & $(40,1 \%)$ & $61 / 87$ & $(70,1 \%)$ & $+30,0 \%$ \\
\hline kennen & $5 / 61$ & $(8,2 \%)$ & $8 / 18$ & $(44,4 \%)$ & $+36,2 \%$ \\
\hline müssen & $118 / 381$ & $(31,0 \%)$ & $57 / 83$ & $(68,7 \%)$ & $+37,7 \%$ \\
\hline heißen & $32 / 132$ & $(24,2 \%)$ & $15 / 23$ & $(65,2 \%)$ & $+41,0 \%$ \\
\hline wissen & $15 / 107$ & $(14,0 \%)$ & $19 / 33$ & $(57,6 \%)$ & $+43,6 \%$ \\
\hline finden & $5 / 32$ & $(15,6 \%)$ & $41 / 53$ & $(77,4 \%)$ & $+61,8 \%$ \\
\hline
\end{tabular}

Bei einigen Verben (sollen, stehen und sitzen) verwendet die jüngere Gruppe das Präteritum etwas seltener als die ältere Gruppe. Ein Chi-Quadrat-Test zeigt allerdings, dass diese Unterschiede nicht-signifikant sind. Bei den restlichen Verben gebraucht die jüngere Sprechergruppe zum Teil deutlich häufiger die Präteritumform. Während der Unterschied beim Verb sein mit 7,6\% vergleichsweise gering ist, verwenden die jüngeren Sprecher die Präteritumform von finden mit 77,4\% vs. 15,6\% extrem viel häufiger als die älteren Sprecher. Der Grund, warum der Unterschied zwischen jüngeren und älteren Sprechern bei sein vergleichsweise gering ist, könnte sein, dass dieses Verb mit $88,3 \%$ von der älteren Gruppe bereits sehr häufig gebraucht wird. Eine weitere Steigerung kann dementsprechend nicht mehr so stark ausfallen.

Anders ist es bei Verben, die von der älteren Gruppe vergleichsweise selten im Präteritum gebraucht werden. Bei den Verben kennen, müssen, heißen, wissen 
und finden, die von der älteren Gruppe zwischen 8,2\% und 31,0\% im Präteritum gebraucht werden, steigt die relative Verwendung des Präteritums zwischen $36,2 \%$ und $61,8 \%$.

Es lässt sich festhalten, dass die relative Frequenz des Präteritums aller Verben, die im Gesamtkorpus mindestens zweimal gebraucht werden, vom SSA zu REDI+FLARS (dt.) deutlich zugenommen hat. Die relative Frequenz der Präteritalformen des Verbs sagen ist auch in REDI+FLARS (dt.) extrem gering, sodass durchaus angezweifelt werden kann, dass die Präteritalform dieses Verbs tatsächlich Verwendung findet. Die restlichen 22 Verben sind dagegen mit relativen Frequenzen des Präteritums zwischen 7,9\% und 87,8\% zweifellos in Gebrauch. Der Vergleich des älteren Korpus SSA und des jüngeren Korpus REDI+FLARS (dt.) bestätigt auf den ersten Blick die bereits in Kapitel 3.1.3 aufgestellte Vermutung, dass die Frequenz des Präteritums in REDI+FLARS (dt.) umso größer ist, je größer sie im SSA ist. Schließt man allerdings die besonders stark korrelierenden Verben sein und sollen aus, so zeigt die Korrelationsanalyse keinen signifikanten Zusammenhang zwischen den Verben im SSA und in REDI+FLARS (dt.) an. Auch der Apparent-Time-Vergleich der älteren mit der jüngeren Sprechergruppe in REDI+FLARS (dt.) zeigt bei den meisten Verben einen deutlichen Anstieg der Präteritumfrequenz. Der Sprachwandel, der durch den Real-Time-Vergleich der Korpora SSA und REDI+FLARS (dt.) ermittelt werden konnte, scheint sich auch beim Apparent-Time-Vergleich der älteren mit der jüngeren Sprechergruppe in REDI+FLARS (dt.) fortzusetzen.

Kapitel 3.1.6 stellt nun die Formenbestände der beiden Korpora gegenüber und geht der Frage nach, welche Verben präteritumaffin sind und welche Gemeinsamkeiten diese Verben haben.

\subsubsection{Formeninventar des Präteritums}

In diesem Kapitel stelle ich das Formeninventar in den Korpora SSA und REDI+FLARS (dt.) vor. ${ }^{17}$ Tabelle 12 dient zur Erklärung der Symbole aus Tabelle 13.

17 Teile dieses Unterkapitels wurden mit einer Teilmenge der Daten bereits in Leonhard (2021) publiziert. 
Tab. 12: Erklärung der verwendeten Symbole in Tabelle 13

\begin{tabular}{ll}
\hline+ & Präteritumform ist belegt \\
\hline$(+)$ & Präteritumform ist kaum belegt (seltener als 1\% im Vergleich \\
& zur Perfektform) \\
- & Präteritumform ist nicht belegt \\
\hline
\end{tabular}

Die nachfolgende Tabelle 13 präsentiert die Verben, bei denen mindestens zwei Präteritum-Belege in mindestens einem der beiden Korpora belegt sind. Der Vergleich der beiden Korpora zeigt, dass die Anzahl der unterschiedlichen Verben im Untersuchungszeitraum zugenommen hat. Während im SSA für 15 Verben (und für drei weitere sehr selten) Präteritum belegt ist, sind in REDI+FLARS (dt.) 22 Verben (und eines sehr selten) im Präteritum nachweisbar. Die Präteritalform des Verbs sagen ist dabei aufgrund der sehr geringen Belegzahl von zwei und einem relativen Anteil von 0,1\% als idiolektale Form zweier Sprecher zu bewerten und wird daher nicht näher behandelt.

Tab. 13: Formenbestand der präteritumfähigen Verben in den Korpora SSA und REDI+FLARS (dt.)

\begin{tabular}{lll}
\hline & SSA & REDI+FLARS (dt.) \\
\hline aussehen & + & + \\
denken & - & + \\
finden & + & + \\
geben & + & + \\
gehen & + & + \\
haben & $(+)$ & + \\
halten & - & + \\
heißen & + & + \\
kennen & - & + \\
kommen & + & + \\
lassen & - & + \\
liegen & + & + \\
sagen & - & ++ \\
sitzen & + & + \\
stehen & + & + \\
werden & + & + \\
\hline
\end{tabular}




\begin{tabular}{lll}
\hline & SSA & REDI+FLARS (dt.) \\
\hline wissen & $(+)$ & + \\
dürfen & + & + \\
können & + & + \\
müssen & $(+)$ & + \\
sollen & + & + \\
wollen & + & + \\
sein & + & + \\
Anzahl der verschiedenen & $15(18)$ & $22(23)$ \\
Verben & & \\
\hline
\end{tabular}

Auffällig ist, dass alle präteritumaffinen Verben stark oder irregulär und zumindest einigermaßen frequent sind. Die Frequenz alleine scheint dabei kein hinreichendes Kriterium für die Präteritumbildung zu sein, da von den höchstfrequenten Verben sagen und machen (29807 bzw. 11708 Belege in den Korpora SSA und REDI+FLARS (dt.) $)^{18}$ kein Präteritum gebildet wird. Zudem wird deutlich, dass die meisten der 22 Verben, die im Korpus REDI+FLARS (dt.) im Präteritum gebrauchten werden, States sind (vgl. Kap. 2.3.2). Das in Kapitel 2.3.2 dargestellte Schema zur Einteilung der Verben in verschiedene Aktionsarten definiert States als durative, nicht-dynamische und atelische Verben. Das heißt, Zustandsverben haben eine zeitliche Ausdehnung (=durativ); die mit dem Verb beschriebene Situation führt keine Veränderung herbei, benötigt keinen Energieaufwand (=nicht-dynamisch) und hat keinen Grenzpunkt (=atelisch), die Handlung ist also auf kein Ende gerichtet (anders als beispielsweise bei aufstehen oder einschlafen). Diese drei Eigenschaften treffen auf die Mehrzahl der 22 präteritumaffinen Verben im Korpus REDI+FLARS (dt.) zu, weshalb sie als Zustandsverben bezeichnet werden können. Zu diesen zählen die Verben aussehen, denken, haben, halten, kennen, lassen, liegen, sitzen, stehen, wissen, dürfen, können, müssen, sollen, wollen und sein.

Daneben finden sich in obiger Liste (vgl. Tabelle 13) auch Verben, die per se keine Zustandsverben sind. Das sind finden, geben, gehen, heißen, kommen und werden. Allerdings haben diese Verben (mit Ausnahme des Verbs werden) stative Teilbedeutungen. Ich möchte im Folgenden die verschiedenen Teilbedeutungen der Verben, die im Korpus REDI+FLARS (dt.) vorkommen, vorstellen und aufzeigen, welche Aktionsart sie haben. Dabei präsentiere ich auch die quantitative

18 Die Modalverben sollen und wollen haben dagegen nur eine Beleghäufigkeit von 2239 bzw. 4391 in den beiden Korpora. 
Verteilung der Präteritalformen auf die verschiedenen Aktionsarten beim jeweiligen Verb. Aufgrund der Vielzahl an Bedeutungen bei einigen Verben habe ich versucht, sehr ähnliche Bedeutungen zusammenzufassen. Grundlegend dafür war, dass sich die Bedeutungen nur minimal voneinander unterscheiden und derselben Aktionsart zuzuordnen sind. Beim Verb werden ist keine stative Teilbedeutung festzustellen. Dieses Verb bildet allerdings insofern eine Ausnahme, da - wie in Kapitel 3.1.5 bereits beschrieben - 92,3\% der Belege zur Passivbildung verwendet werden. In Kapitel 3.2.6 werde ich die Passivbildung durch die Verben sein und werden hinsichtlich Perfekt und Präteritum vergleichen.

\section{finden}

Beim Verb finden lassen sich im Wesentlichen zwei Bedeutungen unterscheiden: zum einen die Bedeutung '(nach einer Suche) auf etwas treffen oder stoßen' („Nach längerer Suche finde ich das Haus“) und zum anderen die Bedeutung 'etwas auf eine bestimmte Weise beurteilen oder empfinden' („Ich finde das Haus schön“). Zunächst präsentiere ich ein Beispiel, bei dem das Verb finden die erste Bedeutung ausdrückt, danach eines, bei dem die zweite Bedeutung zu sehen ist.

In Beispiel (28) fordert der Explorator EX1_1052 die Gewährsperson GP1_1064 auf, ihr Dialektgebiet auf einer Karte einzuzeichnen (Z. 01).

(28) Bombach (REDI+FLARS (dt.))

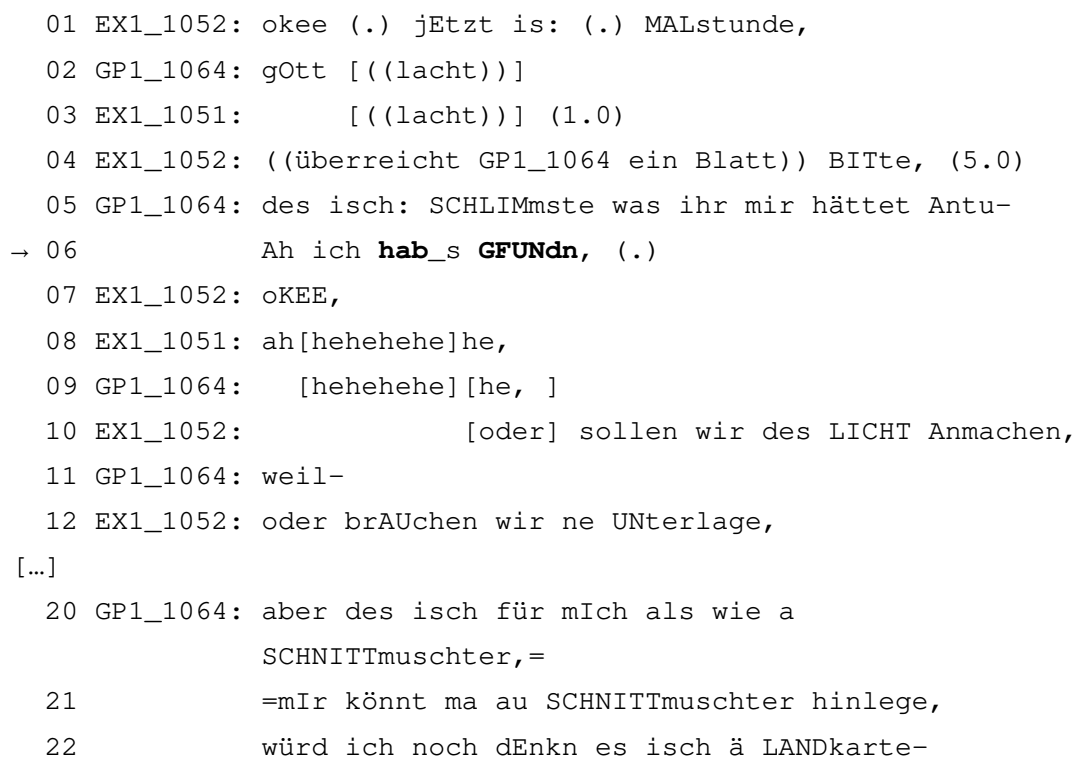




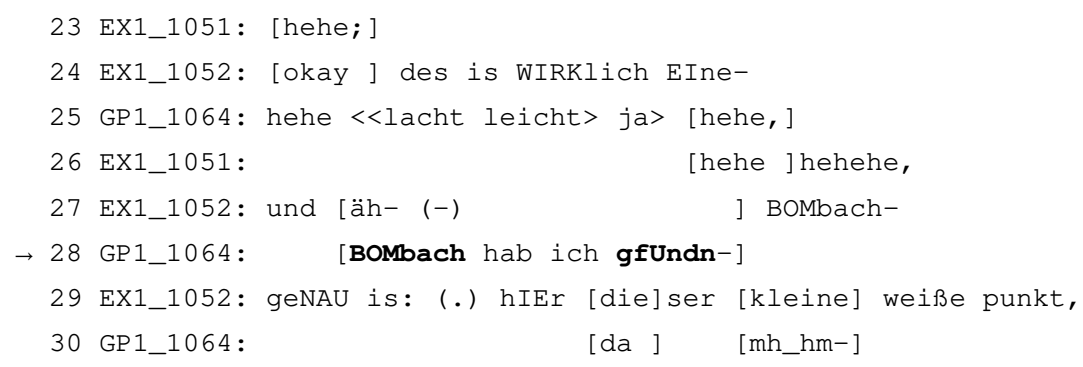

EX1_1052 überreicht GP1_1064 eine Karte (Z. 03), auf der weiße Punkte ohne Beschriftung eingezeichnet sind (Z. 29). Einer dieser Punkte ist der Heimatort der Gewährsperson (Bombach), der ihr bei der Einzeichnung des Dialektgebiets als Orientierung dienen soll (Z. 27-29). In Zeile 05 äußert sie ihre Probleme mit Karten. Das change-of-state Token „Ah“ (vgl. Heritage 1984) in Zeile 06 zeigt ein Verstehen oder Auffinden an, das die Sprecherin in Zeile 06 explizit macht, indem sie sagt, sie habe Bombach gefunden. In den Zeilen 20-22 betont sie noch einmal, dass sie Schwierigkeiten mit Karten habe. Der Explorator EX1_1052 möchte ihr in den Zeilen 27 und 29 Bombach als Orientierungspunkt zeigen. GP1_1064 erklärt parallel dazu, dass sie Bombach bereits gefunden habe (Z. 28). Das Verb finden wird in den Zeilen 06 und 28 in der Bedeutung '(nach einer Suche) auf etwas stoßen/treffen' gebraucht. Wie bereits in Kapitel 2.3.2 beschrieben handelt es sich beim Verb finden in dieser Bedeutung um die Aktionsart Achievement.

In Beispiel (29) fragt der Explorator EX1_1052 die Gewährsperson GP1_1075, welcher Dialekt ihr am wenigsten gefalle (Z. 01).

(29) Breisach (REDI+FLARS (dt.))

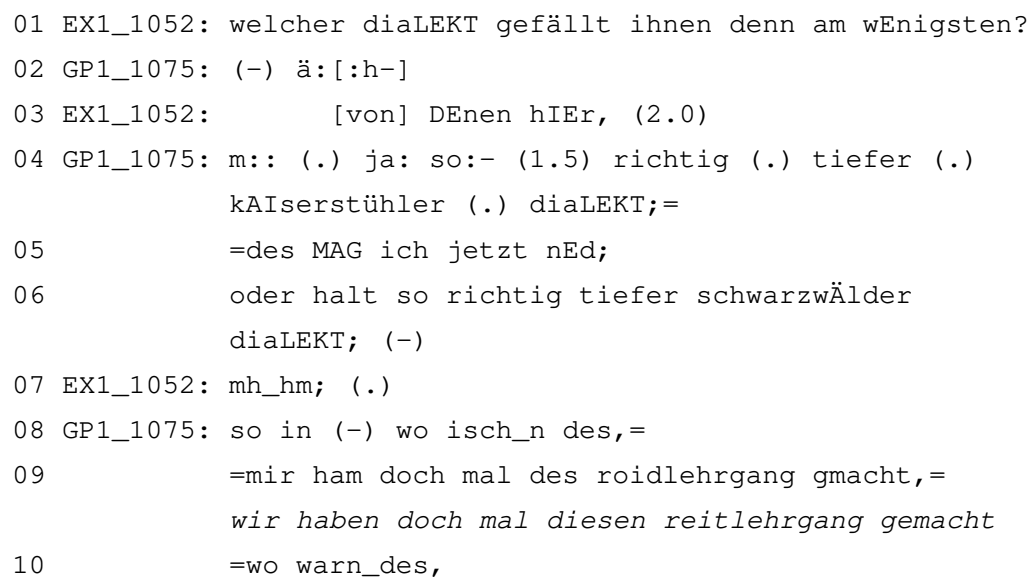




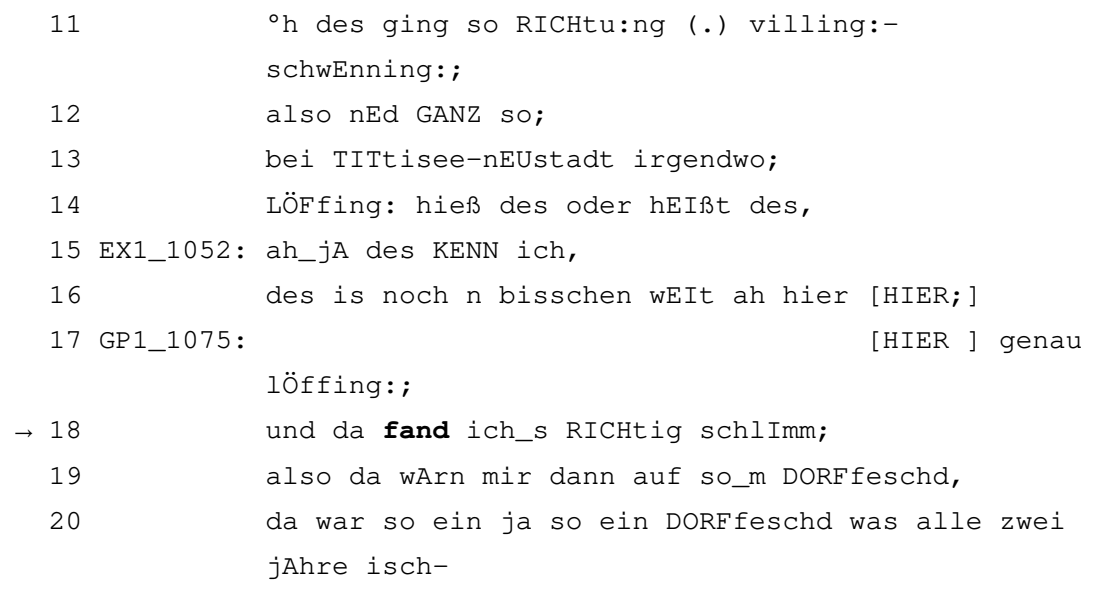

Die Sprecherin antwortet auf diese Frage, sie möge sehr starken Kaiserstühler und Schwarzwälder Dialekt nicht (Z. 04-06). Ab Zeile 08 initiiert sie eine Spezifizierung. Nach einer längeren Wortsuche nach dem Namen eines Ortes (Z. 08-13) nennt sie Löffingen (Z. 14) und erzählt, dass sie es dort „RICHtig schlImm“ (Z. 18) fand. Das Verb finden drückt hier anders als in Beispiel (28) nicht den erfolgreichen Abschluss einer Suche aus, sondern die Einschätzung einer Sache (hier eines Dialekts). Mit ihrer Äußerung in Zeile 18 beantwortet die Gewährsperson GP1_1075 die von EX1_1052 in Zeile 01 gestellte Frage nach ihrer Einschätzung zum unbeliebtesten Dialekt. Im Gegensatz zu Beispiel (28) ist das Verb finden hier um eine adverbiale Gruppe („RICHtig schlImm“) erweitert. Anders als bei der Bedeutung 'auf etwas stoßen' ist bei der Bedeutung 'etwas einschätzen' eine solche adverbiale Gruppe nicht fakultativ, sondern obligatorisch. Dementsprechend lassen sich bei allen Verwendungen des Verbs finden mit dieser Bedeutung solche Prädikativergänzungen feststellen. Die Bedeutung 'etwas einschätzen' ist als State zu interpretieren: Die hier ausgedrückte Situation ist andauernd (=durativ), es wird keine Veränderung herbeigeführt (=nicht-dynamisch) und sie ist nicht auf ein Ziel hin ausgerichtet (=atelisch).

Der quantitative Vergleich der Präteritalformen zeigt, dass alle 46 Belege des Verbs finden im Präteritum die Bedeutung 'etwas einschätzen' haben und somit als States zu beurteilen sind.

\section{geben}

Beim Verb geben sind in meinem Korpus drei Bedeutungen auszumachen: 1.) die Bedeutung 'jemandem etwas überreichen’ („Ich gebe dir das Buch“), 2.) die 
Bedeutung ‘ergeben’ (,Zwei plus zwei (er)gibt vier“) und 3.) ‘existieren’ („Es gab viele Bücher in der Bücherei“).

Beispiel (30) zeigt eine Verwendung des Verbs geben mit der Bedeutung 'jemandem etwas überreichen'. Hier fragt die Exploratorin EX1_1051 nach der Katze der Gewährsperson GP2_1069.

(30) Herbolzheim (REDI+FLARS (dt.))

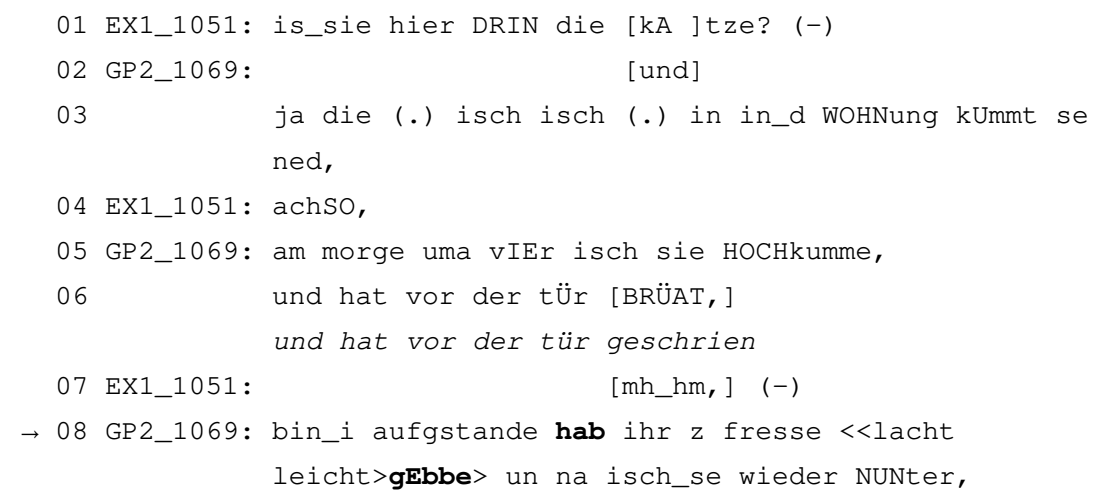

Im Vorfeld dieses Ausschnitts erzählt GP2_1069 von seiner Katze. Daraufhin fragt EX1_1051, ob sie in der Wohnung sei (Z. 01). GP2_1069 antwortet, die Katze komme nicht in die Wohnung (Z. 02). Morgens sei sie vor die Tür gekommen und habe geschrien (Z. 05-06), woraufhin er ihr etwas $\mathrm{zu}$ fressen gegeben habe (Z. 08). Das Verb geben hat hier die Bedeutung 'jemandem etwas überreichen'. Formal wird das durch die Argumentstruktur mit Subjekt (,i“), Dativobjekt (,ihr“) und Akkusativobjekt ((etwas) „z fresse“) deutlich (Z. 08); semantisch dadurch, dass GP2_1069 mit seiner Äußerung in Zeile 08 ausdrückt, dass er seiner Katze das Essen 'überreicht'. Diskutabel ist hierbei, ob es sich aktionsartlich um ein Achievement oder ein Accomplishment handelt. Beide Aktionsarten sind dynamisch und telisch: Geben erfordert einen Energieaufwand und führt eine Veränderung der Situation herbei (=dynamisch) und ist auf ein Ziel hin ausgerichtet (=telisch). GP2_1069 verändert die Situation, da die Katze durch sein Handeln Essen erhält. Zielorientiert ist seine Handlung, da sie auf einen erfolgreichen Abschluss ausgerichtet ist (der Katze etwas zu fressen $\mathrm{zu}$ geben). Fraglich ist dagegen, ob der Vorgang durativ oder nicht-durativ ist. Betrachtet man ihn als durativ, so ist die Verbalhandlung als Accomplishment zu betrachten, wird sie als nicht-durativ angesehen, ist es ein Achievement. Die Frage, ob man den Vorgang als durativ oder nicht-durativ erachtet, ist damit verbunden, ob bei geben der singuläre Moment gemeint ist, in dem der Besitz eines Gegenstandes von 
Lebewesen A zu Lebewesen B wechselt, oder ob darunter der komplette Vorgang verstanden wird, der zum Überreichen einer Sache erforderlich ist (dazu gehören in diesem konkreten Fall unter anderem das Holen des Futters sowie das Befüllen und Bereitstellen des Napfes etc.).

Eine Verwendung des Verbs geben, die klar als nicht-durativ und damit als Achievement anzusehen ist, zeigt Beispiel (31).

(31) Kork (REDI+FLARS (dt.))

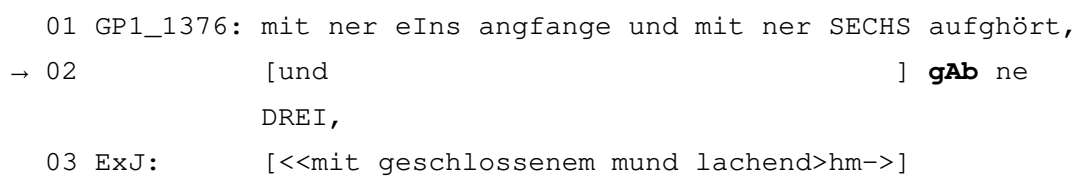

Die Exploratorin ExJ erzählt im Vorfeld dieses Ausschnitts, sie habe Physik mit der Note vier abgeschlossen. Daraufhin erwidert die Gewährsperson GP1_1376, sie habe Physik mit der Note drei abgeschlossen. Sie habe eine eins und eine sechs erhalten (Z. 01), was eine drei ergebe (Z. 02). Geben hat hier die Bedeutung 'zum Ergebnis haben'. Eine zeitliche Ausdehnung ist hier nicht zu sehen. Verrechnet man die Note sechs mit der Note eins, entsteht augenblicklich die Note drei. Anders als in der Bedeutung 'jemandem etwas überreichen' fehlt hier ein Dativobjekt.

In Beispiel (32) erzählt die Gewährsperson GP1_1110 vom Turnunterricht aus ihrer Jugend.

(32) Bötzingen (REDI+FLARS (dt.))

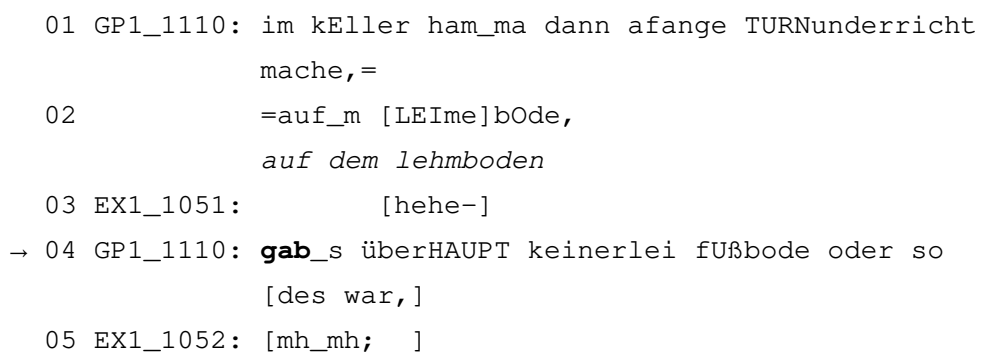

Damals haben sie den Turnunterricht im Keller auf dem Lehmboden machen müssen (Z. 01-02), da es dort keinen Fußboden gab (Z. 05). Das Verb geben hat hier die Bedeutung 'existieren'. Formal lässt sich ein Unterschied zu den beiden vorherigen Bedeutungen an dem Expletivum („es“) (beziehungsweise hier in 
getilgter Form „_s“(Z. 04)), dem Akkusativobjekt (,keinerlei fUßbode“) und dem fehlenden Dativobjekt erkennen. Semantisch ist die Bedeutung 'existieren' daran ersichtlich, dass hier ein Austausch mit existieren möglich ist:

(33) a. Es gab überhaupt keinerlei Fußboden.

b. Es existierte überhaupt keinerlei Fußboden.

Aktionsartlich ist diese Bedeutung als State zu sehen, da die hier ausgedrückte Situation ohne zeitliche Begrenzung ist (=durativ), keine Veränderung herbeigeführt wird (=nicht-dynamisch) und nicht auf ein Ziel hin ausgerichtet ist (=atelisch). Beschrieben wird ein unveränderter Zustand.

Der quantitative Vergleich offenbart, dass 226 der 227 Präteritalformen des Verbs geben als State zu beurteilen sind (vgl. Beispiel (32)). Ein Beleg (vgl. Beispiel (31)) ist ein Achievement.

Tab. 14: Verteilung der Präteritalformen des Verbs geben nach der Aktionsart

\begin{tabular}{llllll}
\hline \multicolumn{2}{l}{ Achievement } & State & \multicolumn{3}{c}{ Gesamt } \\
\hline 1 & $(0,44 \%)$ & 226 & $(99,6 \%)$ & 227 & $(100 \%)$ \\
\hline
\end{tabular}

\section{gehen}

Im Korpus REDI+FLARS (dt.) finden sich für das Verb gehen in einem Vergangenheitstempus fünf verschiedene Bedeutungen. Das Verb losgehen hat zwei Bedeutungen ('sich physisch in Bewegung setzen' und 'beginnen'):

1. 'von A nach B bewegen'

2. 'zur Schule gehen/die Schule besuchen'

3. 'funktionieren/möglich sein'

4. 'sich bis zu einem (Zeit-/Orts-)Punkt erstrecken'

5. 'Thema sein'

In Beispiel (34) ist die Verwendung des Verbs losgehen in der Bedeutung 'anfangen/beginnen’ zu sehen. Die Sprecherin erzählt hier vom Beginn des Zweiten Weltkriegs.

(34) Bötzingen (REDI+FLARS (dt.))

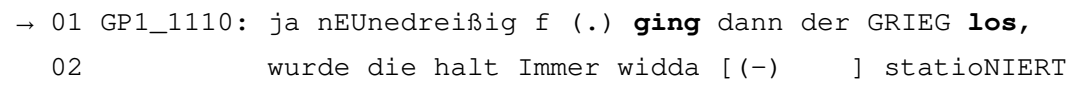


und so,

03 EX1_1051:

04 EX1_1052:

$$
\begin{array}{r}
{\left[\mathrm{mh}_{-}[\mathrm{hm},]\right.} \\
{[\mathrm{hm}-]}
\end{array}
$$

Belege mit dieser Bedeutung sind als Achievement zu kategorisieren. Der Moment, in dem der Krieg beginnt, besitzt keine zeitliche Ausdehnung. Es gibt lediglich ein vor dem Krieg und ein nach Beginn des Krieges. Aus diesem Grund ist diese Verbbedeutung nicht-durativ. Da eine Veränderung der Situation herbeigeführt und ein Energieaufwand notwendig ist, ist das Verb dynamisch. Gleichzeitig ist die Verbalsituation zielorientiert (auf den Krieg) und somit telisch.

Beispiel (35) illustriert die Verwendung des Verbs gehen in seiner Bedeutung 'von A nach B bewegen'. Die Gewährsperson GP1_1162 erzählt hier von der Rivalität benachbarter Orte mit unterschiedlicher Konfession.

(35) Herrischried (REDI+FLARS (dt.))

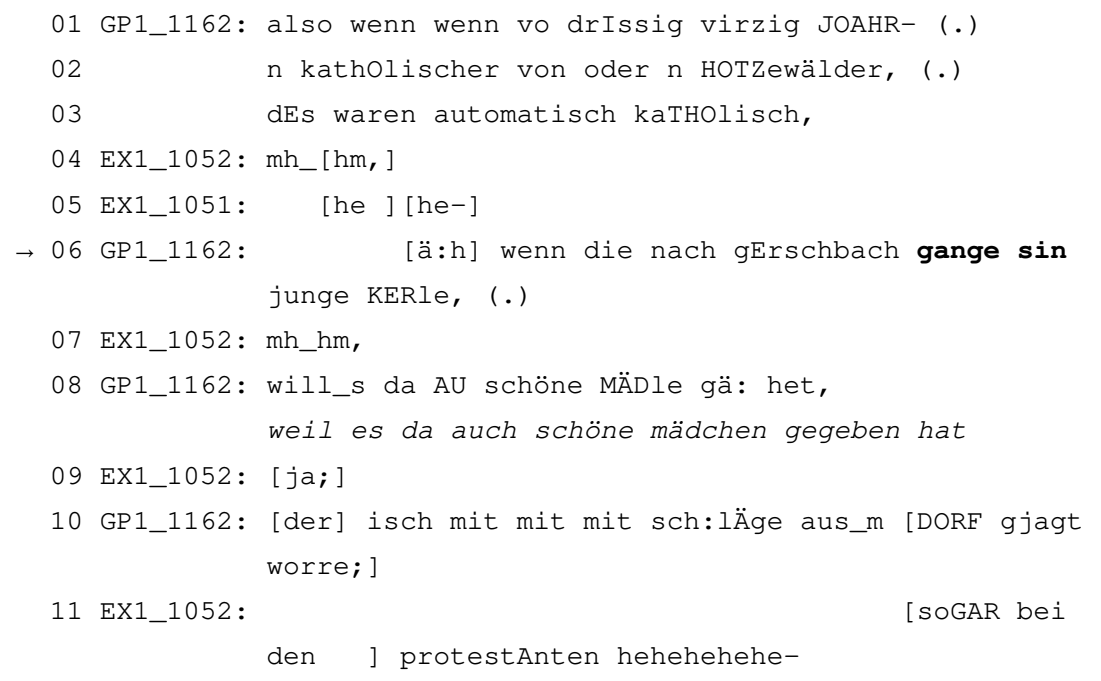

GP1_1162 berichtet, wenn Katholiken früher in seinen Ortsteil Gersbach gekommen seien (Z. 01-06), wären sie mit Schlägen aus dem Dorf gejagt worden (Z. 10). Das Verb gehen hat hier die Bedeutung 'von A nach B bewegen'. Semantisch wird hier die tatsächliche Bewegung von einem katholischen Ort beziehungsweise dem Hotzenwald nach Gersbach thematisiert. Formal werden entsprechend die obligatorischen Argumente Subjekt („die“) und Adverbialergänzung („nach gErschbach") realisiert. Das fakultative Argument von A ist hier zwar nicht realisiert, es lässt sich aber leicht als vom Hotzenwald ergänzen. Aktionsartlich 
handelt es sich bei dieser Bedeutung um ein Accomplishment. Die Verbalsituation weist hierbei eine zeitliche Ausdehnung auf (=durativ), sie führt eine Veränderung herbei, da das Subjekt seine Position verändert (=dynamisch), und sie ist auf ein Ziel (in diesem Fall Gersbach) ausgerichtet (=telisch).

In Beispiel (36) berichten die Gewährspersonen GP1_1189 und GP2_1189 von einem Nachbarsjungen, der für sie unerklärlicherweise standarddeutsch spricht.

(36) Malsburg (REDI+FLARS (dt.))

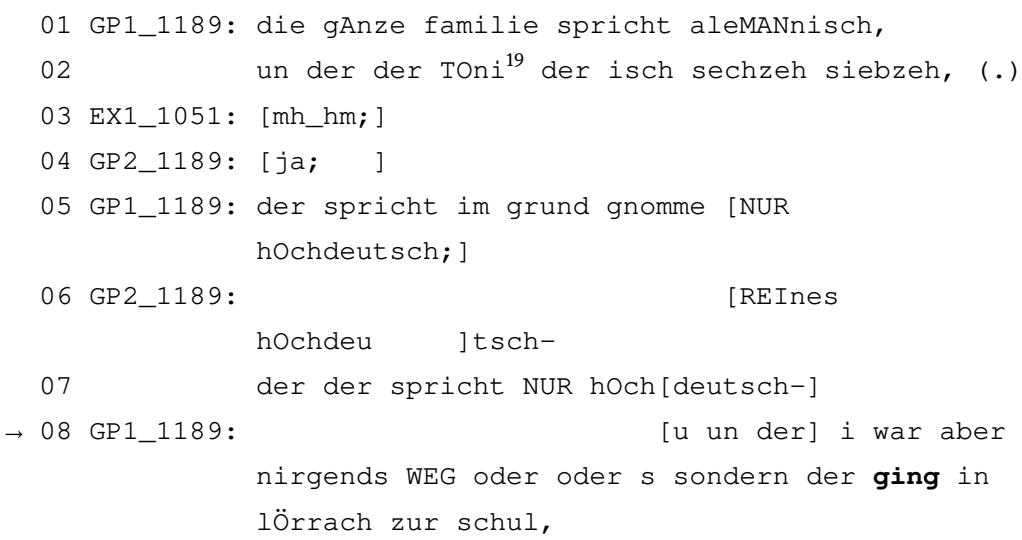

In Zeile 01-05 erzählt GP1_1189, ihr Nachbarsjunge Toni sei der einzige in der Familie, der nicht Alemannisch spreche, sondern „NUR hOchdeutsch“ (Z. 05). Nach der Bekräftigung durch GP2_1189 (Z. 06) expandiert GP1_1189 seine Erzählung in Zeile 08 und nimmt einen möglichen Einwand vorweg und fügt hinzu, Toni habe nie den Ort verlassen und gehe im circa 20 Kilometer entfernten Lörrach zur Schule. Die Bedeutung des Verbs gehen lässt sich hier nur mit der Prädikativergänzung „zur schul“ (Z. 08) erschließen. Neben dieser Prädikativergänzung sind auch ein Subjekt („der“) und eine Adverbialergänzung (,in lÖrrach“) obligatorische Argumente. Semantisch wird hier keine Bewegung ausgedrückt, sondern ein Zustand (der des Besuchens der Schule). Dementsprechend fordert die Präposition ,in“ in diesem Fall nicht den Akkusativ, der eine Richtung anzeigen würde, sondern den Dativ, der auf eine Position hinweist. Vergleiche dazu die Sätze (37):

19 In den Transkripten wurden ausschließlich Pseudonyme gewählt, die in der Silbenlänge und in der Namensherkunft den Originalnamen entsprechen. 
(37) a. Der Ballon schwebt über die Brücke.

b. Der Ballon schwebt über der Brücke.

Das Verb gehen in dieser Bedeutung ist der Aktionsart State zuzuordnen. Der Schulbesuch weist keine zeitliche Begrenzung auf (=durativ), er führt keine Veränderung herbei (=nicht-dynamisch) und ist nicht auf ein Ziel hin ausgerichtet (=atelisch).

Die Sprecherin GP1_1337 berichtet in Beispiel (38) von ihren sprachlichen Gewohnheiten in ihrer Arbeit.

(38) Freistett (REDI+FLARS (dt.))

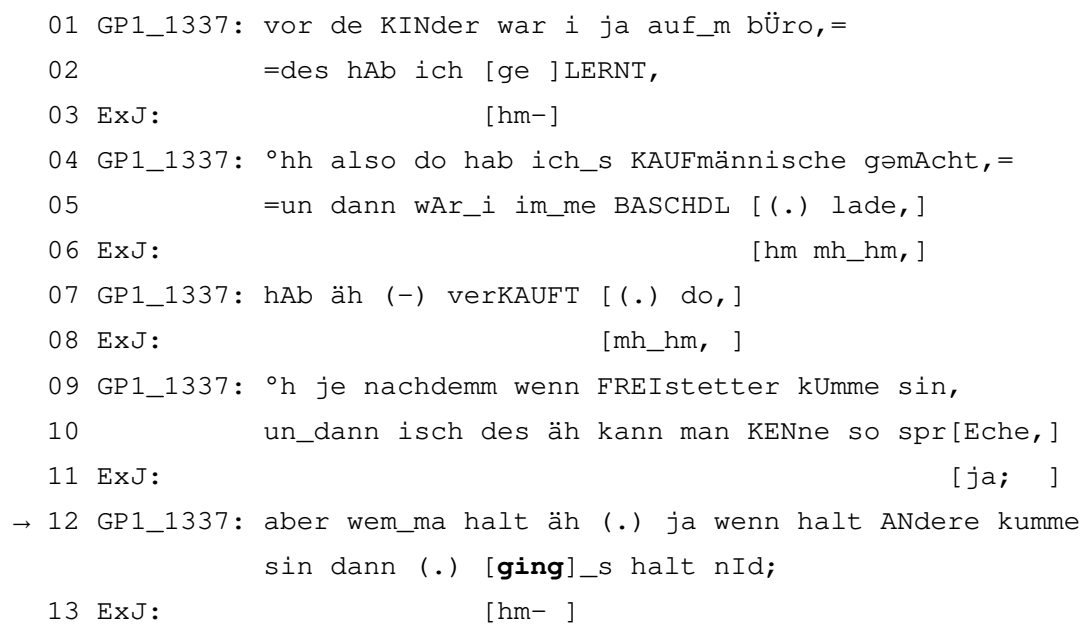

Im Vorfeld dieses Ausschnitts fragt die Exploratorin ExJ, ob sich die Sprachgewohnheiten der Gewährsperson im Laufe der Zeit verändert haben. GP1_1337 erzählt daraufhin von ihrer Arbeit in einem Bastelladen in Freistett (ab Z. 05). Wenn Freistetter dort eingekauft hätten, habe sie ihren eigenen Dialekt sprechen können (Z. 09-10). Bei Auswärtigen sei das nicht gegangen (Z. 12). Das Verb gehen hat hier die Bedeutung 'möglich sein'. Morpho-syntaktisch fordert gehen hier lediglich ein Subjekt. Es wird keine Bewegung beschrieben, sondern ausgedrückt, ob etwas möglich ist. Aus diesem Grund könnte die Konstruktion „ging_s halt nId“ (Z. 12) durch war_s halt nid möglich ersetzt werden. Auch diese Bedeutung ist als State zu betrachten, da es sich um einen andauernden und unbegrenzten Zustand handelt (=durativ), ohne inhärente Veränderung (=nicht-dynamisch) und auf kein Ziel gerichtet (=atelisch). 
In Beispiel (39) thematisiert die Gewährsperson GP1_1217 den unterschiedlichen Dialektalitätsgrad zwischen Städtern und Dorfbewohnern (Z. 01-03).

(39) Oberried (REDI+FLARS (dt.))

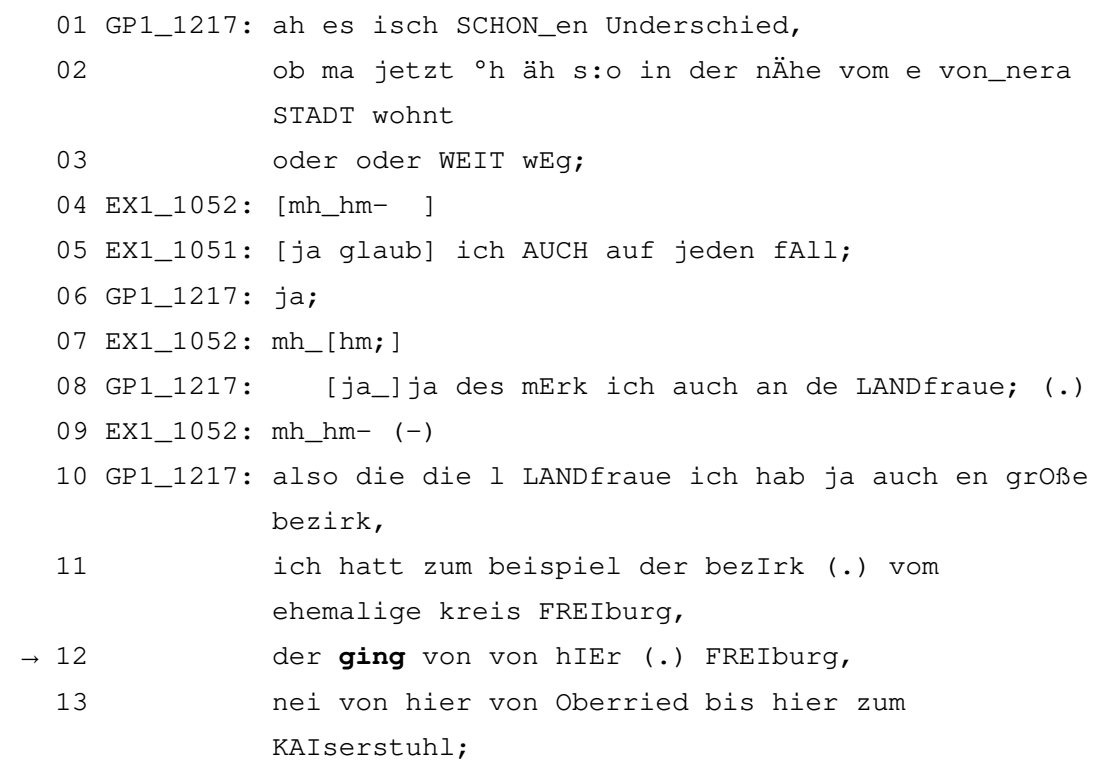

Nachdem GP1_1217 erklärt hat, dass es einen Unterschied mache, ob man in einer Stadt wohnt oder weiter davon weg (Z. 01-03), expandiert sie ihre Einschätzung ab Zeile 08 mit einem Beispiel über Landfrauen. Vor ihrer Einschätzung, wie sehr diese Landfrauen Dialekt sprechen, beschreibt sie die Größe des Bezirks, in dem sie als Meisterin der ländlichen Hauswirtschaft zuständig war (Z. 10-11). Dieser erstreckte sich „,vom ehemalige kreis FREIburg“ (Z. 11) bis „zum KAIserstuhl“ (Z. 13). Das Verb gehen in Zeile 12 drückt auch hier keine Bewegung von einem Punkt zu einem anderen aus, sondern ist synonym zu den Verben sich erstrecken oder sich ausdehnen. Dementsprechend fordert es die obligatorischen Argumente Subjekt („der“) und Adverbialergänzung (,bis hier zum KAIserstuhl“) sowie das fakultative Argument Adverbialergänzung (,von hIEr (.) FREIburg“) (Z. 12-13). Die Aktionsart des Verbs gehen ist in dieser Bedeutung ein State. Die Beschreibung der Größe des Bezirks ist permanent (=durativ), ohne Veränderung (=nicht-dynamisch) und nicht zielorientiert (=atelisch).

Beispiel (40) zeigt die Verwendung des Verbs gehen in der Bedeutung 'Thema sein'. Der Sprecher GP1_1086 erzählt hier von seinem im Krieg verschollenen Vater. 
(40) Waldkirch (REDI+FLARS (dt.))

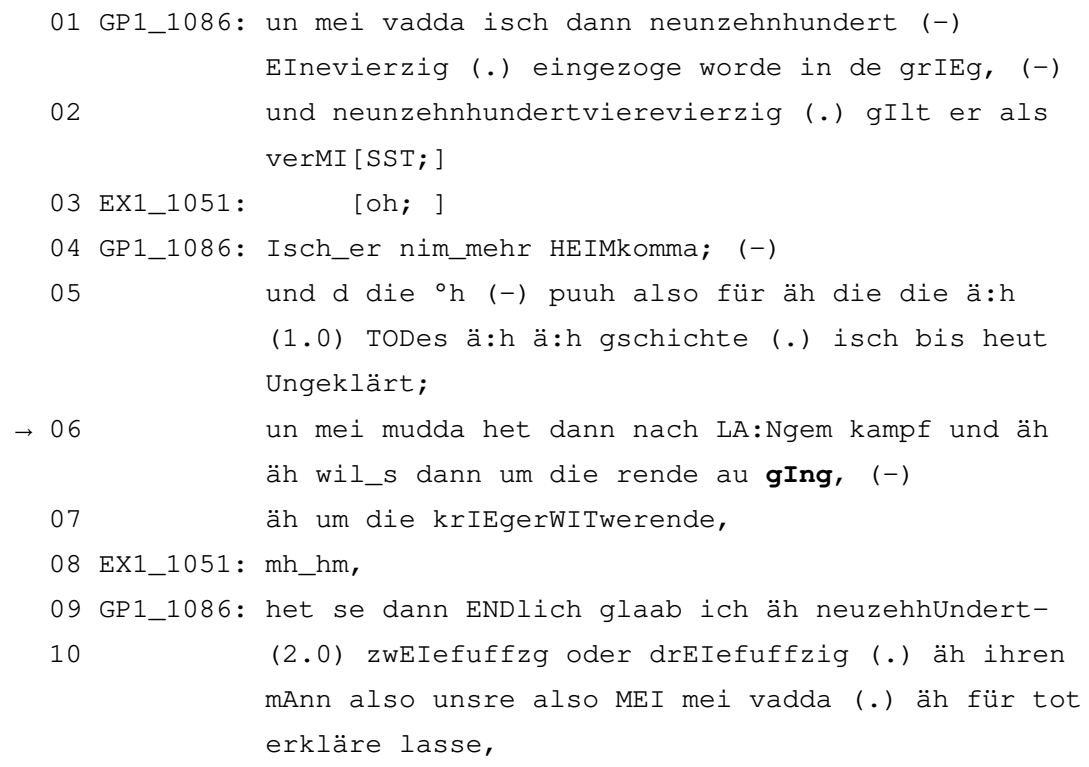

1941 sei der Vater von GP1_1086 eingezogen (Z. 01) und 1944 als vermisst gemeldet worden (Z. 02). Da die Todesumstände bis heute ungeklärt gewesen seien ( $Z$. 05), habe seine Mutter ihn erst 1952 oder 1953 für tot erklären lassen (Z. 10), um Anspruch auf die Kriegerwitwenrente zu erhalten (Z. 06-07). Das Verb gehen in Zeile 06 drückt hierbei die Bedeutung 'Thema sein' aus. Die Äußerungen dann ging es um die Rente und dann war die Rente das Thema sind also austauschbar. In dieser Bedeutung fordert gehen ein Scheinsubjekt (,,s“) und eine Prädikativergänzung („um die rende“). Es handelt sich dabei um die Aktionsart State, da die Verbalsituation durativ, nicht-dynamisch und atelisch ist.

Ein quantitativer Vergleich der Präteritalformen des Verbs gehen für die verschiedenen Aktionsarten zeigt, dass mit 74,3\% die große Mehrheit der Belege States sind. 6,9\% sind als Achievement zu bewerten. Diese gehen allesamt auf das Verb losgehen (sowohl in der Bedeutung des konkreten Beginns des Laufens als auch in der Bedeutung des abstrakten Beginnens) zurück. Accomplishments, also die eigentliche Grundbedeutung des Verbs gehen, machen nur 27 der 144 Präteritalformen aus (18,8\%). 
Tab. 15: Verteilung der Präteritalformen des Verbs gehen nach der Aktionsart

\begin{tabular}{|c|c|c|c|c|c|c|c|}
\hline \multicolumn{2}{|c|}{ Accomplishment } & \multicolumn{2}{|c|}{ Achievement } & \multirow{2}{*}{$\frac{\text { State }}{107}$} & \multicolumn{3}{|c|}{ Gesamt } \\
\hline 27 & $(18,8 \%)$ & 10 & $(6,9 \%)$ & & $(74,3 \%)$ & 144 & $(100 \%)$ \\
\hline
\end{tabular}

\section{heißen}

Das Verb heißen weist im Korpus REDI+FLARS (dt.) zwei Bedeutungen auf. Zum einen die Bedeutung 'einen Namen tragen' („Sie heißt Michaela“) und zum anderen die Bedeutung 'gesagt werden' („Dann hieß es, wir sollten um zehn im Bett sein").

In Beispiel (41) erzählt die Gewährsperson GP1_1068 von einer Wallfahrtskirche, die „kaPELleli“ (Z. 12) hieß.

(41) Herbolzheim (REDI+FLARS (dt.))

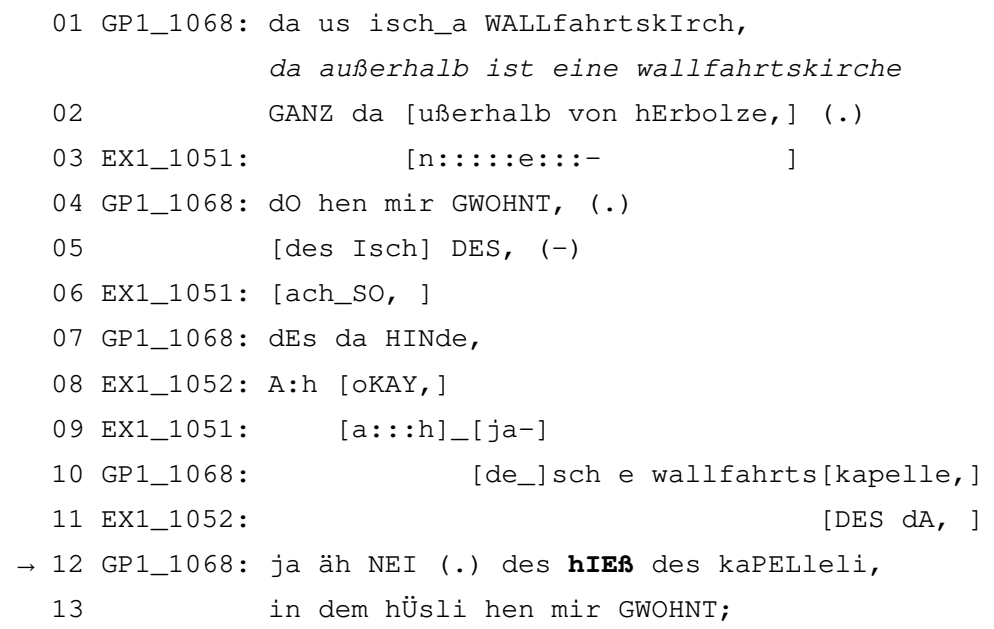

Diese Bedeutung ist als State zu beurteilen, da sie eine zeitliche (unbegrenzte) Ausdehnung besitzt (=durativ), nicht zielorientiert ist (=atelisch) und kein Energieaufwand nötig ist (=nicht-dynamisch).

Die Verwendung des Verbs heißen in Beispiel (42) hat die Bedeutung 'gesagt werden'. Hier erzählt der Sprecher GP1_1343 von Vorurteilen zwischen den benachbarten Orten Greffern und Lichtenau. 
(42) Greffern (REDI+FLARS (dt.))

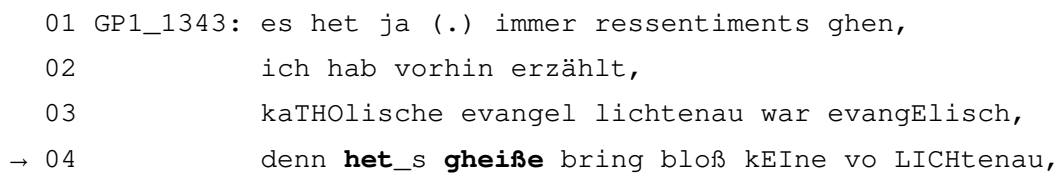

Aufgrund verschiedener Konfessionen in Greffern und Lichtenau (Z. 03) habe es Vorurteile in Greffern gegeben (Z. 01). Daher sollte man keine Liebesbeziehung mit einer Frau aus Lichtenau eingehen (Z. 04). Das Verb heißen drückt hier nicht den Namen von etwas oder jemandem aus, sondern verbalisiert eine wiederkehrende Äußerung. Die Gesamtperspektive ist durchaus als durativ und damit unabgeschlossen einzustufen, aktionsartlich dagegen besitzt die Verbalhandlung (fast) keine zeitliche Ausdehnung. ${ }^{20}$ Die Verbalhandlung wird also über eine unbegrenzte Zeitdauer immer wieder singulär geäußert. Jede einzelne Verbalhandlung ist nicht-durativ (darauf bezieht sich die Aktionsart), die gesamte Äußerung (,denn het_s gheiße bring bloß kEIne vo LICHtenau“ Z. 04) ist dagegen andauernd. Die Verbalhandlung selbst ist dynamisch (d. h. sie benötigt einen Energieaufwand), aber nicht zielorientiert (sie markiert also nicht das Ende oder den Beginn einer Situation). Aus diesem Grund ist die Verbbedeutung 'gesagt werden' als Semelfactive anzusehen.

Insgesamt machen States mit 38 (80,9\%) gegenüber neun Belegen (19,1\%) den Großteil aller Verwendungen beim Verb heißen im Präteritum aus.

Tab. 16: Verteilung der Präteritalformen des Verbs heißen nach der Aktionsart

\begin{tabular}{llllll}
\hline \multicolumn{2}{l}{ Achievement } & State & \multicolumn{3}{l}{ Gesamt } \\
\hline 9 & $(19,1 \%)$ & 38 & $(80,9 \%)$ & 47 & $(100 \%)$ \\
\hline
\end{tabular}

\section{kommen}

Aufgrund der Vielzahl an Bedeutungen, die beim Verb kommen im Korpus REDI+FLARS (dt.) zu finden sind, beschränkt sich die folgende Aufstellung auf die Bedeutungen, die in Präteritalformen ausgedrückt werden. Es lassen sich dabei folgende Bedeutungen unterscheiden:

1. 'von A nach B gelangen'

20 Zum Zusammenspiel von Aktionsart und Aspekt beziehungsweise (Un-)Abgeschlossenheit der Situation siehe auch Kap. 2.2.3. 
2. 'Teil von etwas werden'

3. 'in Erscheinung treten/sich ereignen/eröffnet/erfunden werden'

4. 'irgendwo sein'

5. 'seinen Grund/Ursprung in etwas haben'

In Beispiel (43) erzählt die Gewährsperson GP1_1324, dass sie schon als Kind nie nach Frankreich gefahren sei (Z. 01).

(43) Auenheim (REDI+FLARS (dt.))

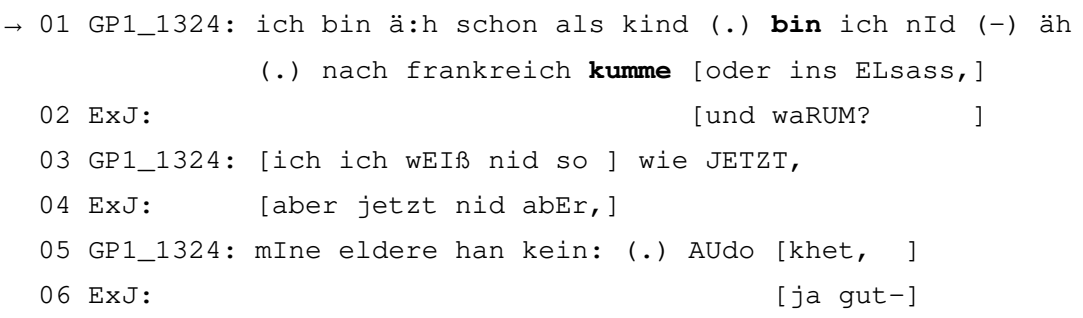

Auenheim liegt direkt an der deutsch-französischen Grenze. Dennoch gibt GP1_1324 an, sie sei schon als Kind nicht nach Frankreich gekommen (Z. 01). Auf die Nachfrage der Exploratorin ExJ in Zeile 02 erklärt GP1_1324 zunächst, sie wisse nicht warum (Z. 03). Begründet es dann allerdings damit, dass ihre Eltern kein Auto gehabt hätten (Z. 05). Die Exploratorin ExJ akzeptiert diese Erklärung in Zeile 06. Kommen hat hier die Bedeutung 'von A nach B gelangen'. Die morphosyntaktische Valenz umfasst ein Subjekt (,ich“), eine obligatorische Adverbialergänzung (,nach frankreich“) und eine hier nicht realisierte, fakultative Adverbialergänzung (von a). Semantisch wird hier eine Bewegung ausgedrückt. Dies wird unter anderem daran deutlich, dass als Grund für die fehlende Bewegung das nicht vorhandene Auto (also ein Fortbewegungsmittel) angeführt wird. Aktionsartlich ist diese Verbbedeutung ein Accomplishment. Die Verbalhandlung ist zeitlich ausgedehnt (=durativ), erfordert einen Energieaufwand (=dynamisch) und ist auf ein Ziel hin ausgerichtet (=telisch).

Die Gewährsperson GP1_1413 berichtet in Beispiel (44) von ihrer Bewerbung als Lehrer.

(44) Offenburg (REDI+FLARS (dt.))

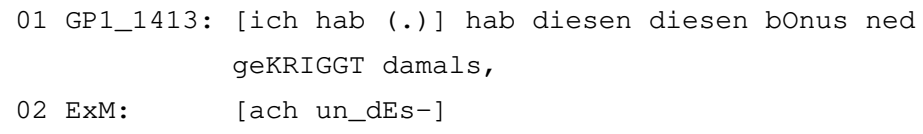




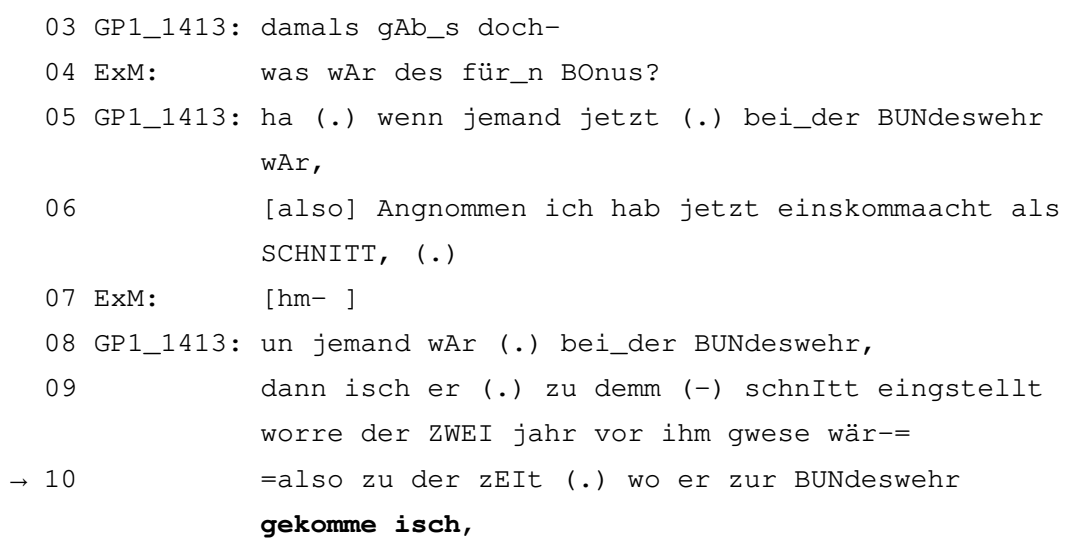

Für die Bewerbung als Lehrer habe es für Männer, die Wehrdienst geleistet haben (Z. 08), einen Bonus gegeben (Z. 01), d. h. einen verbesserten Notenschnitt ( $Z$. 09-10). GP1_1413 selbst habe diesen Bonus allerdings nicht erhalten (Z. 01). In Zeile 10 terminiert er den Schnitt genauer: Es sei der Schnitt zu der Zeit, zu der jemand zur Bundeswehr gekommen sei. Die Bedeutung des Verbs kommen ist hier der in Beispiel (43) dargestellten sehr ähnlich. Auch in Beispiel (44) steht das Ziel im Vordergrund (=telisch) und es ist ein Energieaufwand notwendig (=dynamisch). Allerdings wird hier keine konkrete Bewegung ausgedrückt, bei der sich jemand oder etwas von einem Punkt zu einem anderen bewegt, sondern lediglich der Moment erfasst, bei dem jemand Teil von etwas wird (=nicht-durativ). Dementsprechend ist auch die fakultative Adverbialergänzung, die den Ursprung der Bewegung ausdrückt, nicht vorhanden (und auch nicht ergänzbar). Als Aktionsart lässt sich diese Bedeutung den Achievements zuordnen.

Beispiel (45) präsentiert einen Fall für eine Verwendung von kommen mit der Bedeutung 'in Erscheinung treten/sich ereignen/eröffnet/erfunden werden'. Der Sprecher GP1_97 erzählt hier von seinen Aufzeichnungen über strukturelle Veränderungen durch die Post, Telefon und die Eisenbahnerfindung (Z. 03).

(45) Altheim bei Riedlingen (SSA)

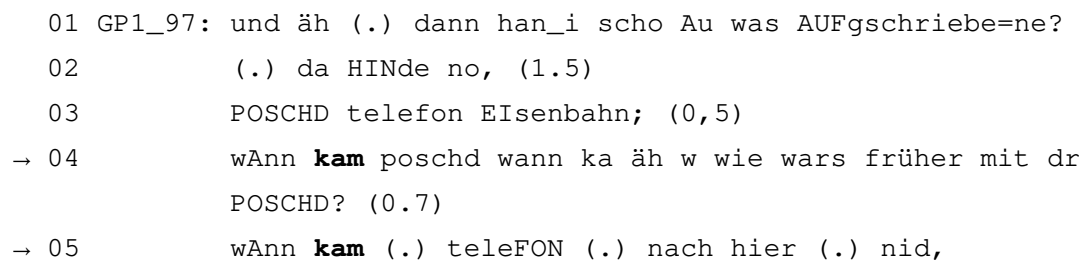


GP1_97 erzählt, er habe aufgeschrieben (Z. 01), wann die Post (Z. 04) und wann das Telefon kam (Z. 05). Kommen drückt hierbei keine Bewegung im eigentlichen Sinn aus, sondern das Erscheinen von etwas. Post und Telefon bewegen sich nicht nach Altheim, sondern werden dort eröffnet beziehungsweise dorthin transportiert. Das Verb fordert hier neben einem Subjekt (,poschd“ (Z. 04) beziehungsweise „teleFON“ (Z. 05)) nur eine fakultative Adverbialergänzung (nach $x$ ), die in der Äußerung in Zeile 05 („,nach hier“) realisiert ist. Diese Verbbedeutung ähnelt in einigen Punkten der bei Beispiel (44) beschriebenen. Auch diese ist als Achievement zu klassifizieren, da die Verbalhandlung keine zeitliche Ausdehnung besitzt, d. h. kein Prozess beschrieben wird, sondern das Vorhandensein des Subjekts in ein Davor und ein Danach unterteilbar ist (=nicht-durativ), einen Energieaufwand benötigt und eine Veränderung der Situation erzeugt wird (=dynamisch) und zielorientiert (=telisch) ist.

In Beispiel (46) berichtet der Sprecher GP1_1112 von seinem Urlaub an der Nordsee.

(46) Bötzingen (REDI+FLARS (dt.))

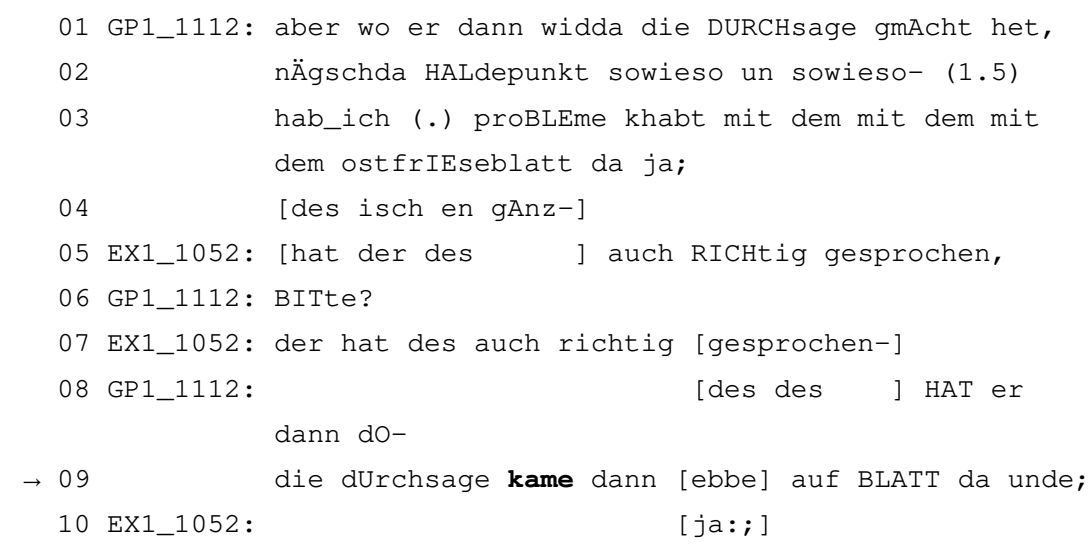

Im Vorfeld dieses Ausschnitts erzählt GP1_1112, er sei mit einem Reisebus an die Nordsee gefahren. Dort habe er sich mit dem aus Norddeutschland stammenden Busfahrer unterhalten und ihn gut verstanden. Die Durchsagen des Busfahrers seien allerdings auf Plattdeutsch gewesen, sodass er ihn nur noch schwer verstanden habe (Z. 01-03). Kommen in Zeile 09 drückt anders als die eben beschriebenen Situationen keine dynamische Verbalhandlung aus, sondern einen $\mathrm{Zu}$ stand. Das ist morpho-syntaktisch an den fehlenden Adverbialergänzungen des Ursprungs und des Ziels zu erkennen. Semantisch lässt sich der Zustand durch die Austauschbarkeit des Verbs kommen durch sein feststellen: 
(47) a. Die Durchsagen kamen auf Platt.

b. Die Durchsagen waren auf Platt.

Die Äußerung die Durchsagen kamen auf Platt ähnelt also der Verwendung von kommen in Äußerungen wie: Der Film kommt im Kino (vs. Der Film kommt ins Kino). Hierbei kann das Vorhandensein eines Subjekts auch nicht in ein Davor und ein Danach unterteilt werden, wie dies in Beispiel (45) möglich ist. Stattdessen wird ein zeitlich unbegrenzter Zustand beschrieben. Aus diesem Grund ist die Verbbedeutung in (46) als durativ, nicht-dynamisch und atelisch zu betrachten und damit aktionsartlich als State.

Die Sprecherin GP1_1324 schildert in Beispiel (48) einen Aufenthalt in Köln, bei dem sie als Kind Verständigungsschwierigkeiten hatte.

(48) Auenheim (REDI+FLARS (dt.))

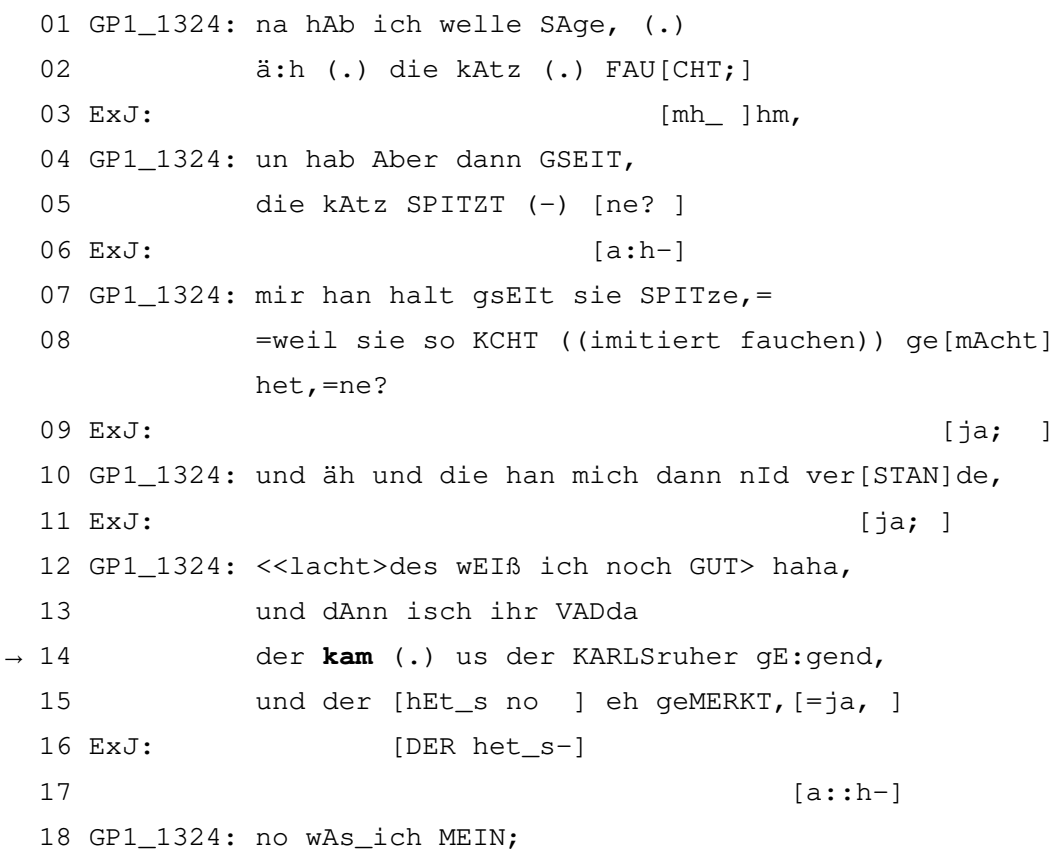

In den Zeilen 01-05 berichtet GP1_1324, sie habe bei ihrem Besuch in Köln anstelle des standardsprachlichen Ausdrucks „FAUCHT“ (Z. 02) die niederalemannische Bezeichnung „SPITZT“ (Z. 05) verwendet. Sie expandiert diese Äußerung um eine Erklärung des dialektalen Wortes in den Zeilen 07-08. Daraufhin erzählt sie, dass sie nicht verstanden wurde (Z. 10). Lediglich der Vater einer Freundin 
habe sie verstanden (Z. 13-18). Der Grund, warum er sie verstanden hatte, wird in Zeile 14 angegeben („der kam (.) us der KARLSruher gE:gend“). Auch wenn eine Bewegung von einem Ort zu einem anderen mitgedacht wird (hier also von der Karlsruher Gegend nach Köln), steht hier der Ursprung einer Person im Vordergrund. Das wird morpho-syntaktisch daran deutlich, dass eine die Richtung anzeigende Adverbialergänzung (nach $x$ ) fehlt und eine den Ursprung anzeigende Adverbialergänzung (,us der KARLSruher gE:gend“ (Z. 14)) obligatorisch ist. Im Gegensatz dazu ist bei der Verbbedeutung ‘von A nach B gelangen’ die den Ursprung anzeigende Adverbialergänzung fakultativ und die das Ziel anzeigende Adverbialergänzung obligatorisch. Semantisch wird die Ursprungsbedeutung in Beispiel (48) daran ersichtlich, dass der Ursprung als Begründung herangezogen wird. Eine Begründung für das Verständnis des Niederalemannischen wäre mit einer Bewegung nicht möglich. Aus diesem Grund könnte die Äußerung „der kam (.) us der KARLSruher gE:gend“ durch die Äußerung der war us der Karlsruher Gegend ersetzt werden, allerdings nicht durch die Äußerung der hat sich us der Karlsruher Gegend (hier her) bewegt. Die Bedeutung 'seinen Grund/Ursprung in etwas haben' ist dementsprechend als State zu betrachten. Es handelt sich um eine irreversible Eigenschaft, d. h. einen durativen Zustand, der keinen Energieaufwand benötigt oder eine Veränderung herbeiführt (=nicht-dynamisch) und nicht zielorientiert ist (=atelisch).

Der Vergleich der Aktionsarten für die Präteritalformen des Verbs kommen ergibt ein weniger klares Bild als bei den bisherigen Verben. Achievements sind mit 17 Belegen $(17,2 \%)$ deutlich unterrepräsentiert. Demgegenüber sind Accomplishments mit 109 Belegen (46,0\%) und States mit 111 Belegen (46,8\%) in etwa gleich häufig. Die Präteritalformen des Verbs kommen sind also deutlich seltener States als die der bisher betrachteten Verben.

Tab. 17: Verteilung der Präteritalformen des Verbs kommen nach der Aktionsart

\begin{tabular}{llllllll}
\hline \multicolumn{2}{l}{ Accomplishment } & \multicolumn{2}{l}{ Achievement } & \multicolumn{2}{l}{ State } & \multicolumn{2}{l}{ Gesamt } \\
\hline 109 & $(46,0 \%)$ & 17 & $(7,2 \%)$ & 111 & $(46,8 \%)$ & 237 & $(100 \%)$ \\
\hline
\end{tabular}

Eine Stichprobe der Perfektformen des Verbs kommen zeigt demgegenüber eine grundlegend andere Verteilung. Bei Perfektformen machen Accomplishments mit $366(80,3 \%)$ die überwiegende Mehrheit aus und States mit 14 Belegen (3,1\%) einen äußerst geringen Anteil. 
Tab. 18: Stichprobenhafte Verteilung der Perfektformen des Verbs kommen nach der Aktionsart

\begin{tabular}{llllllll}
\hline \multicolumn{2}{l}{ Accomplishment } & \multicolumn{2}{l}{ Achievement } & \multicolumn{2}{l}{ State } & \multicolumn{2}{l}{ Gesamt } \\
\hline 366 & $(80,3 \%)$ & 76 & $(16,7 \%)$ & 14 & $(3,1 \%)$ & 456 & $(100 \%)$ \\
\hline
\end{tabular}

Angesichts dessen kann für das Präteritum des Verbs kommen eine deutliche Tendenz zu stativen Verbbedeutungen nachgewiesen werden, die allerdings weniger ausgeprägt ist als bei anderen Verben.

Zusammenfassend lässt sich daher festhalten, dass mit Ausnahme des Verbs werden alle Verben, die im Untersuchungskorpus eine Präteritalform aufweisen, entweder Zustandsverben sind oder eine stative Teilbedeutung haben, die im Präteritum gebraucht wird. Das heißt, wir erkennen eine Spezialisierung hinsichtlich der Aktionsart.

\subsubsection{Korrelation zwischen Präteritumgebrauch und Standardnähe}

Es stellt sich die Frage, ob standardnähere Sprecher tendenziell häufiger Präteritum gebrauchen als standardfernere Sprecher. Zwar wäre es denkbar, die Berufsmilieus der Gewährspersonen als Dialektalitätsmarker heranzuziehen, wobei Personen mit kommunikationsorientierten Berufen tendenziell standardnäher sprechen als Personen mit handwerklichen Berufen. Diese Erfassung der Standardnähe erfolgt allerdings indirekt und ist damit etwas fehlerbehaftet. Stattdessen ziehe ich als Marker für Dialektalität für jeden Sprecher die Partizipien der Perfektformen der Verben haben, sagen und sein heran und untersuche das Verhältnis von Standard- und Dialektformen. Produziert ein Sprecher beispielsweise sieben Dialekt- und drei Standardformen so hat er eine relative Standardnähe von 30\%. Diese Methode der Ermittlung der Standardnähe erfolgt ausgehend von den geäußerten Belegen der Sprecher und ist daher unmittelbar und präziser als der Umweg über die Berufsmilieus. Den so ermittelten Dialektalitätsgrad vergleiche ich mit der Präteritumfrequenz der Sprecher, also der relativen Anzahl von Präterital- gegenüber Perfektformen. Zunächst untersuche ich, ob die relative Anzahl der standardnahen gegenüber den standardfernen Partizipien zwischen SSA und REDI+FLARS (dt.) zugenommen hat. Anschließend führe ich eine Korrelationsanalyse nach Pearson durch, um den Dialektalitätsgrad und die Präteritum- 
frequenz der einzelnen Sprecher aus REDI+FLARS (dt.) miteinander zu vergleichen. $^{21}$

Wie in Kapitel 3.1.2 dargestellt habe ich standardnahe Sprechweisen ausgeschlossen. Daher finden sich in den Korpora erwartungsgemäß keine eigentlichen Standardformen dieser Partizipien II. Es lassen sich allerdings standardnahe und standardferne Formen unterscheiden. Als standardferne Formen habe ich die Varianten betrachtet, die im SSA als basisdialektale Varianten angegeben sind. Das sind unter anderem gsat, gsait, gseit für standardsprachliches gesagt (vgl. die SSA-Karte II/30.05), kha, kho, khat, khet für standardsprachliches gehabt (vgl. die SSA-Karte III/1.502) und gsi, gsei, gwen, gwea für standardsprachliches gewesen (vgl. die SSA-Karte III/1.512). Als standardnahe Formen betrachte ich Varianten, die phonologisch näher am Standard sind als die standardfernen Formen: also gsagt, khabt sowie gwese, gewese und gwesen.

Von jedem Sprecher sollten insgesamt zehn Belege ausgewertet werden, wobei auf eine gleichmäßige Verteilung auf die drei Partizipien geachtet wurde. Aufgrund der im bisherigen Kapitel beschriebenen Präteritumexpansion und des damit verbundenen Perfektschwunds fanden sich allerdings (vor allem) in REDI+FLARS (dt.) nicht für alle Sprecher zehn Belege. Besonders die Anzahl des Partizips von sein ist stark zurückgegangen, sodass eine ausgewogene Verteilung der drei Partizipien in REDI+FLARS (dt.) nicht möglich war. Die Partizipien von gewesen machen in diesem Korpus nur 23,0\% aus, die Partizipien von gehabt $33,5 \%$ und die von gesagt 43,6\%. Die vergleichsweise hohe Zahl der Varianten von gesagt erklärt sich durch die fehlende Ausbreitung des Präteritums bei diesem Verb.

Der Vergleich zwischen den beiden Korpora zeigt, dass die relative Zahl der standardfernen Varianten und damit der Dialektalitätsgrad zwischen SSA und REDI+FLARS (dt.) stark abgenommen hat. Während im SSA 3119 (90,7\%) der 3437 Partizipien standardfern sind, sind es in REDI+FLARS (dt.) nur noch 973 (46,3\%) von 2102. Dieses Ergebnis unterstreicht die Validität der hier gewählten Methode zur Ermittlung des Dialektalitätsgrads, da auch in etlichen anderen Untersuchungen ein solcher Rückgang an Dialektalität im Alemannischen festgestellt wurde (vgl. u. a. Streck 2012 und Schwarz 2015). Die nachfolgenden Tabellen 19 und 20 präsentieren die Verteilung der standardnahen und standardfernen Varianten der jeweiligen Partizipien.

21 Da der Dialektalitätsgrad bereits durch die hier vorgestellte Methode ermittelt werden soll, ist eine Unterteilung der Sprecher in eine jüngere und eine ältere Gruppe hierbei nicht nötig. 
Tab. 19: Verteilung der standardnahen und standardfernen Varianten der Partizipien von haben, sagen und sein im SSA

\begin{tabular}{lrrl}
\hline & Standardnah & Standardfern & Gesamt \\
\hline gehabt & $29(2,6 \%)$ & $1074(97,4 \%)$ & $1103(100 \%)$ \\
gesagt & $236(21,0 \%)$ & $890(79,0 \%)$ & $1126(100 \%)$ \\
gewesen & $53(4,4 \%)$ & $1155(95,6 \%)$ & $1208(100 \%)$ \\
Gesamt & $318(9,3 \%)$ & $3119(90,7 \%)$ & $3437(100 \%)$ \\
\hline
\end{tabular}

Tab. 20: Verteilung der standardnahen und standardfernen Varianten der Partizipien von haben, sagen und sein in REDI+FLARS (dt.)

\begin{tabular}{lccc}
\hline & Standardnah & Standardfern & \multicolumn{1}{c}{ Gesamt } \\
\hline gehabt & $251(35,9 \%)$ & $449(64,1 \%)$ & $700(100 \%)$ \\
gesagt & $716(77,7 \%)$ & $206(22,3 \%)$ & $922(100 \%)$ \\
gewesen & $162(33,8 \%)$ & $318(66,3 \%)$ & $480(100 \%)$ \\
Gesamt & $1129(53,7 \%)$ & $973(46,3 \%)$ & $2102(100 \%)$ \\
\hline
\end{tabular}

Der Vergleich der jeweiligen Partizipien zeigt zum einen, dass die standardnahen Varianten der Partizipien zwischen SSA und REDI+FLARS (dt.) deutlich zugenommen haben. Zum anderen offenbart der Vergleich der Partizipien im jeweiligen Korpus große Unterschiede zwischen den Partizipien. Die standardfernen Varianten von gehabt und gewesen sind mit 97,4\% bzw. 95,6\% im SSA und 64,1\% bzw. 66,3\% in REDI+FLARS (dt.) im jeweiligen Korpus etwa gleich verteilt. Die standardfernen Varianten von gesagt weichen mit 79,0\% bzw. 22,3\% davon deutlich ab.

Die folgende Abbildung 7 visualisiert die Verteilung der standardfernen Varianten der drei Partizipien im SSA und in REDI+FLARS (dt.). 


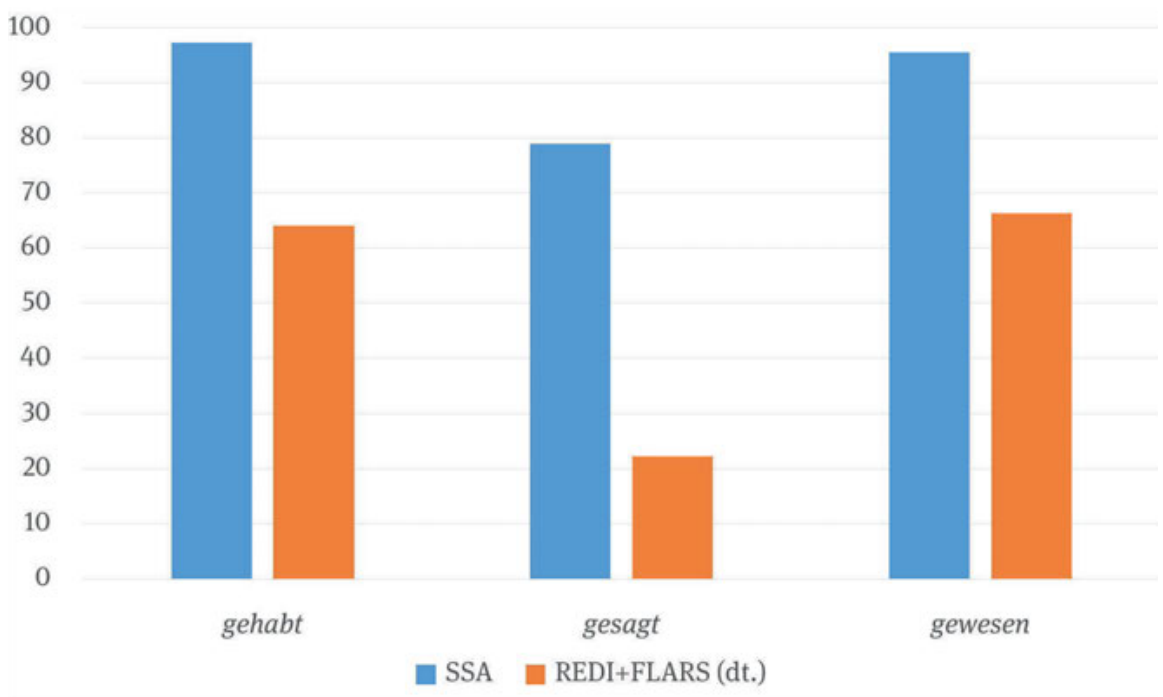

Abb. 7: Relative Häufigkeit der standardfernen Varianten von gehabt, gesagt und gewesen im SSA und in REDI+FLARS (dt.)

Ich möchte nun der Frage nachgehen, ob die Standardnähe und die Häufigkeit der Präteritumverwendung der einzelnen Sprecher in REDI+FLARS (dt.) miteinander korreliert. Da die Partizipien in REDI+FLARS (dt.) nicht gleichmäßig verteilt sind und unterschiedlich häufig als standardnahe beziehungsweise standardferne Variante realisiert werden, müssen sie getrennt voneinander betrachtet werden. Aufgrund der ähnlichen Verteilung der Varianten der Partizipien von gehabt und gewesen können diese gemeinsam behandelt werden. Die Dialektalität wird also doppelt gemessen. Einmal mit den Varianten von gehabt und gewesen und einmal mit denen von gesagt. Für Bestimmung des Dialektalitätsgrads der einzelnen Sprecher wurden nur Gewährspersonen aufgenommen, bei denen mindestens fünf Belege von gehabt/gewesen beziehungsweise gesagt nachweisbar waren. Durch die Trennung der Partizipien waren für einige Sprecher nicht genug Partizipien vorhanden, um sie in die Analyse aufzunehmen. Allerdings stützt die doppelte Messung der Dialektalität die Validität der Ergebnisse.

Verglichen wird diese Dialektalität mit der Präteritumfrequenz der einzelnen Sprecher. In Kapitel 3.1.5 wurde deutlich, dass die präteritumaffinen Verben unterschiedlich häufig im Präteritum vorkommen. Das heißt, die Verben sein und sollen werden mit 90,3\% bzw. 87,8\% deutlich häufiger im Präteritum gebraucht als beispielsweise gehen und haben mit 28,0\% bzw. 22,6\%. Aus diesem Grund ist 
es notwendig, die Verben nach ihrer relativen Präteritumfrequenz zu gruppieren. Für jeden Sprecher sollten für die Präteritumfrequenz mindestens zehn Belege von Präteritum und/oder Perfekt erfasst werden. Die nachfolgende Tabelle 21 illustriert die Gruppen.

Tab. 21: Gruppierung der präteritumaffine Verben nach deren relativer Präteritumfrequenz in REDI+FLARS (dt.)

\begin{tabular}{|c|c|c|c|}
\hline & Verb & Präteritum & Gesamt \\
\hline \multirow[t]{2}{*}{ Gruppe 1} & sein & $6202(90,3 \%)$ & \multirow{2}{*}{$6238(90,3 \%)$} \\
\hline & sollen & $36(87,8 \%)$ & \\
\hline \multirow[t]{5}{*}{ Gruppe 2} & dürfen & $39(58,2 \%)$ & \multirow{5}{*}{$665(51,8 \%)$} \\
\hline & geben & $227(57,5 \%)$ & \\
\hline & finden & $46(52,3 \%)$ & \\
\hline & werden & $169(49,7 \%)$ & \\
\hline & können & $184(46,9 \%)$ & \\
\hline \multirow[t]{9}{*}{ Gruppe 3} & müssen & $180(37,7 \%)$ & \multirow{9}{*}{$1038(25,9 \%)$} \\
\hline & aussehen & $4(36,4 \%)$ & \\
\hline & heißen & $47(29,0 \%)$ & \\
\hline & liegen & $9(28,1 \%)$ & \\
\hline & gehen & $144(28,0 \%)$ & \\
\hline & stehen & $21(25,0 \%)$ & \\
\hline & kommen & $237(24,4 \%)$ & \\
\hline & wissen & $36(23,2 \%)$ & \\
\hline & haben & $360(22,6 \%)$ & \\
\hline \multirow[t]{5}{*}{ Gruppe 4} & kennen & $13(15,9 \%)$ & \multirow{5}{*}{$49(10,4 \%)$} \\
\hline & lassen & $4(13,3 \%)$ & \\
\hline & sitzen & $13(10,5 \%)$ & \\
\hline & denken & 16 (8,0\%) & \\
\hline & halten & $3(7,9 \%)$ & \\
\hline Gruppe 5 & wollen & 277 (74,7\%) & 277 (74,7\%) \\
\hline
\end{tabular}

Die Gruppen 4 und 5 konnten aufgrund der geringen Belegzahl von 49 bzw. 277 nicht zur Analyse herangezogen werden, da kein Sprecher zehn Belege aufwies. Gruppe 2 wurde ebenfalls ausgeschlossen, da in dieser nur 29 Sprecher mindestens zehn Belege produzierten (26 für den Dialektalitätsgrad nach gehabt/gewesen und 25 für den Dialektalitätsgrad nach gesagt). Die Präteritumfrequenz wird 
deshalb mit Hilfe der Gruppen 1 und 3 gemessen. Diese vergleiche ich jeweils mit den Dialektalitätsgraden, die sich durch die Standardnähe der Varianten von gehabt/gewesen und von gesagt ergeben. Somit werden im Folgenden vier Vergleiche gemacht:

1. Dialektalitätsgrad nach gehabt/gewesen mit Präteritumfrequenz von sein und sollen

2. Dialektalitätsgrad nach gesagt mit Präteritumfrequenz von sein und sollen

3. Dialektalitätsgrad nach gehabt/gewesen mit Präteritumfrequenz der Gruppe 3

4. Dialektalitätsgrad nach gesagt mit Präteritumfrequenz der Gruppe 3

5. Standardnähe und Präteritumfrequenz sind in Prozent angegeben, um eine metrische Skala für die Korrelationsanalyse zu ermöglichen.

\section{Dialektalitätsgrad nach gehabt/gewesen mit Präteritumfrequenz von sein und sollen}

Ein Korrelationstest nach Pearson ergibt für die Korrelation des Dialektalitätsgrads, der durch die dialektalen/regionalen Varianten von gehabt und gewesen ermittelt wurde, und der Präteritumfrequenz der Verben sein und sollen einen Wert von 0,329 ${ }^{\star \star \star}(n=126)$ und somit eine schwache Korrelation. Der Korrelationskoeffizient $\mathrm{R}^{2}$ beträgt dementsprechend 0,108.

Der Mittelwert der Standardnähe beträgt 37,2\%, der Mittelwert der Präteritumfrequenz 85,7\%. Die Standardabweichung ist bei der Standardnähe mit 40,5\% sehr hoch, bei der Präteritumfrequenz mit 14,8\% relativ gering. Bei der Standardnähe gibt es also sehr viele Extremwerte, bei der Präteritumfrequenz konzentrieren sich die Werte auf einen relativ geringen Bereich.

Die nachfolgende Abbildung 8 verdeutlicht diese höchstsignifikante, aber schwache Korrelation. Auf der y-Achse liegen die prozentualen Anteile an Präteritalverwendungen gegenüber den Perfektverwendungen der Verben sein und sollen in REDI+FLARS (dt.), auf der x-Achse die relativen Werte der standardnahen Varianten der Partizipien gehabt und gewesen. Es zeigt sich ein linear positiver Zusammenhang zwischen der Präteritumfrequenz und der Standardnähe. 


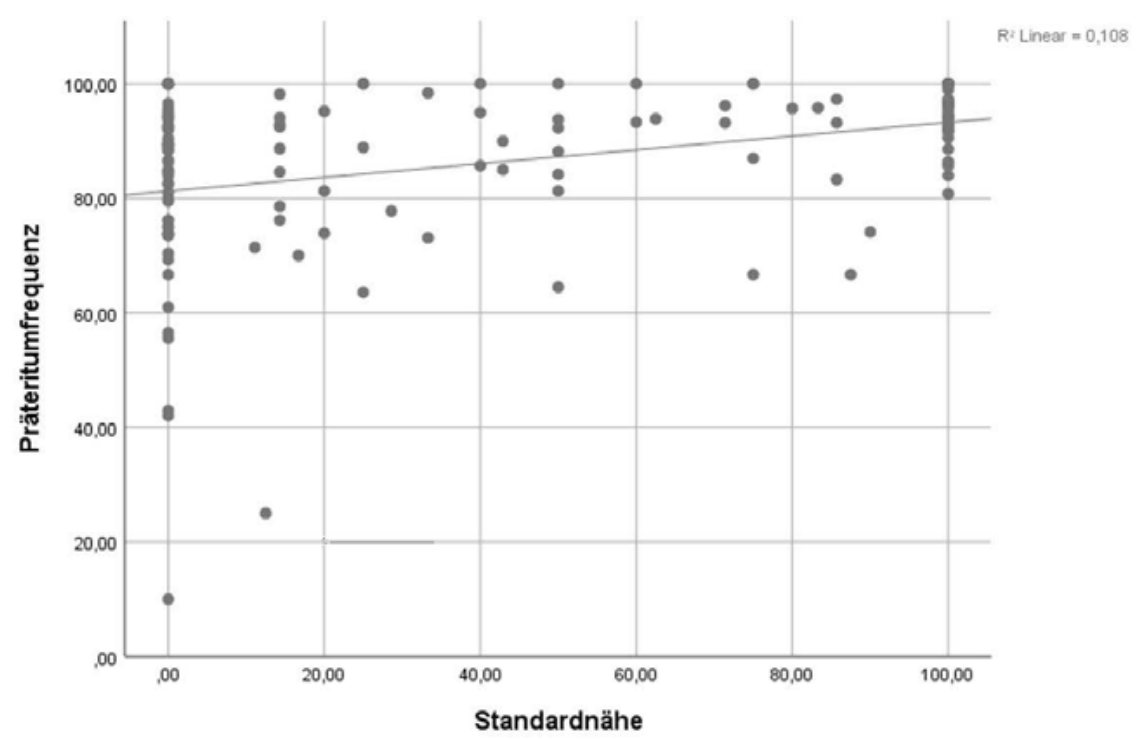

Abb. 8: Einfache Streuung mit Anpassungslinie für den Zusammenhang zwischen Präteritumfrequenz von sein und sollen und Standardnähe nach gehabt/gewesen

\section{Dialektalitätsgrad nach gesagt mit Präteritumfrequenz von sein und sollen}

Die Korrelation der Präteritumfrequenz der Verben sein und sollen und des Dialektalitätsgrads, der mit Hilfe der dialektalen/regionalen Varianten des Partizips gesagt ermittelt wurde, zeigt mit einem Wert von $0,424^{\star \star \star}(n=124)$ eine etwas stärkere Korrelation an, die aber dennoch als schwach zu bewerten ist. Der Korrelationskoeffizient $\mathrm{R}^{2}$ beträgt dementsprechend 0,180. Da nicht bei allen Sprechern sowohl für gehabt/gewesen als auch für gesagt ausreichend Belege nachgewiesen werden konnten, unterscheiden sich die Sprechergruppen für die mit gehabt/gewesen und gesagt bestimmten Dialektalitätsgrade. Dadurch lassen sich die leichten Unterschiede erklären.

Der Mittelwert der Standardnähe beträgt 77,9\%, der Mittelwert der Präteritumfrequenz 93,0\%. Die Standardabweichung ist bei der Standardnähe mit 35,0\% sehr hoch, bei der Präteritumfrequenz mit 7,5\% sehr gering. Bei der Standardnähe gibt es also sehr viele Extremwerte, bei der Präteritumfrequenz konzentrieren sich die Werte auf einen äußerst geringen Teil.

Die nachfolgende Abbildung 9 verdeutlicht diese höchstsignifikante, schwache Korrelation. Auf der y-Achse liegen die prozentualen Anteile an Präteritalverwendungen gegenüber den Perfektverwendungen der Verben sein und sollen in REDI+FLARS (dt.), auf der $\mathrm{x}$-Achse die relativen Werte der standardnahen 
Varianten des Partizips gesagt. Es zeigt sich ein linear positiver Zusammenhang zwischen der Präteritumfrequenz und der Standardnähe.

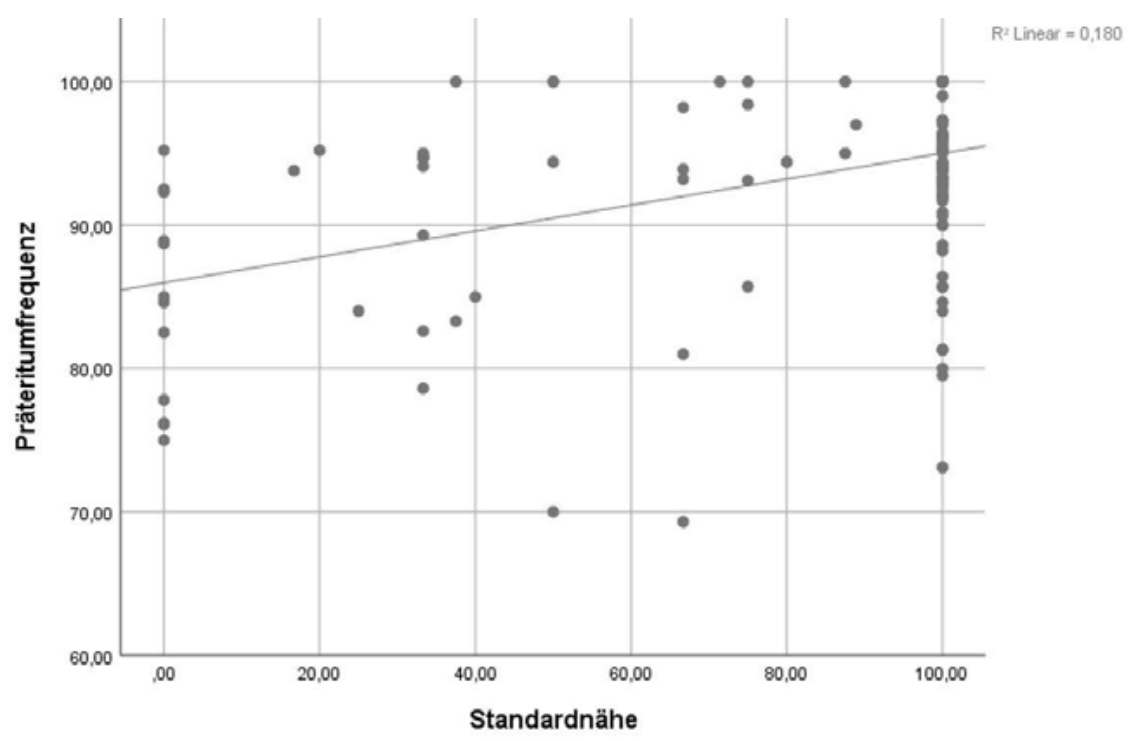

Abb. 9: Einfache Streuung mit Anpassungslinie für den Zusammenhang zwischen Präteritumfrequenz von sein und sollen und Standardnähe nach gesagt

Es wird eine schwache Korrelation der Standardnähe der Varianten von gehabt/gewesen und gesagt und der Präteritumfrequenz von sein und sollen ersichtlich. Standardnähe korreliert also nur bedingt mit einer erhöhten Präteritumfrequenz dieser Verben. Das lässt sich wohl dadurch erklären, dass sein und sollen mit einer relativen Präteritumfrequenz von 90,3\% bzw. 87,8\% von allen Sprechern verwendet werden, unabhängig davon, wie standardnah sie sprechen.

\section{Dialektalitätsgrad nach gehabt/gewesen mit Präteritumfrequenz der Gruppe 3}

Die Korrelation des Dialektalitätsgrads der dialektalen/regionalen Varianten von

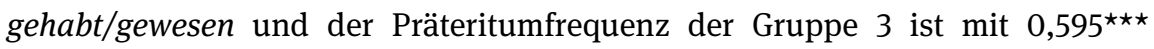
$(\mathrm{n}=110)$ mittelstark. Der Korrelationskoeffizient $\mathrm{R}^{2}$ beträgt dementsprechend 0,353. Der Mittelwert der Standardnähe beträgt 34,6\%, der Mittelwert der Präteritumfrequenz 19,1\%. Die Standardabweichung ist bei der Standardnähe mit 39,7\% sehr hoch, bei der Präteritumfrequenz mit 22,1\% mittelhoch. Bei der Standardnähe gibt es also sehr viele Extremwerte, bei der Präteritumfrequenz 
verteilen sich die Werte auf ein breites Spektrum. Die nachfolgende Abbildung 10 verdeutlicht diese höchstsignifikante, mittelstarke Korrelation. Auf der y-Achse liegen die prozentualen Anteile an Präteritalverwendungen gegenüber den Perfektverwendungen der Verben aus Gruppe 3, auf der x-Achse die relativen Werte der standardnahen Varianten der Partizipien gehabt/gewesen. Es zeigt sich ein linear positiver Zusammenhang zwischen der Präteritumfrequenz und der Standardnähe.

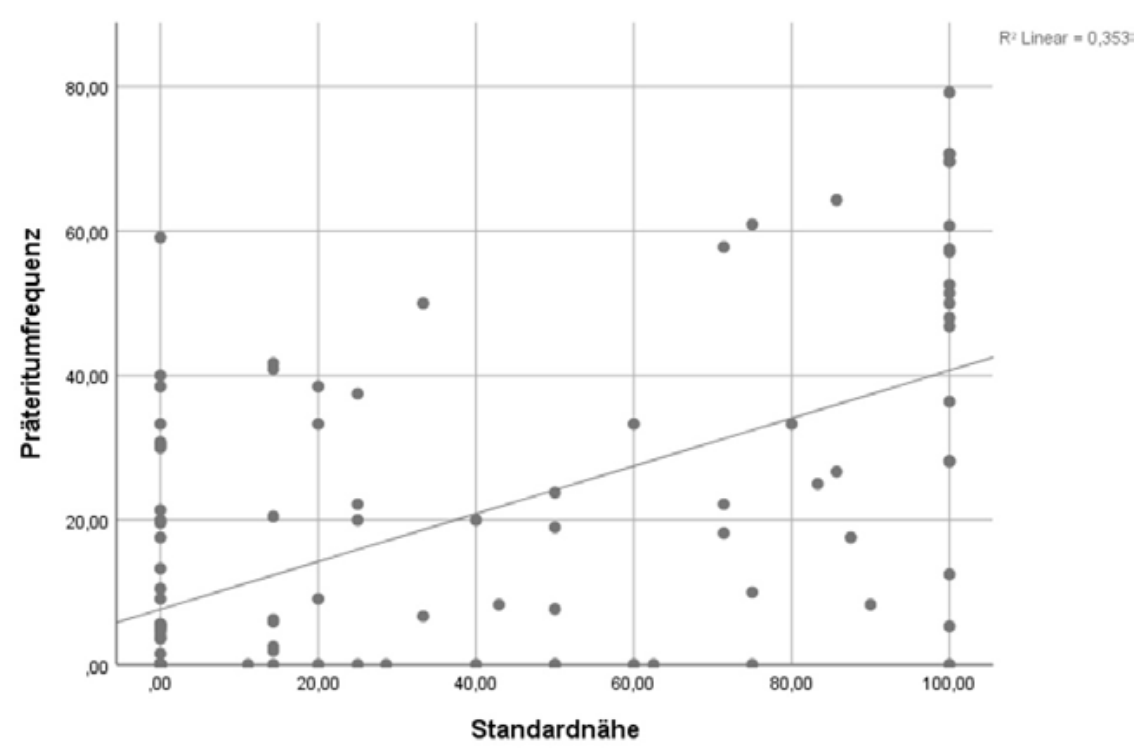

Abb. 10: Einfache Streuung mit Anpassungslinie für den Zusammenhang zwischen Präteritumfrequenz der Gruppe 3 und Standardnähe nach gehabt/gewesen

\section{Dialektalitätsgrad nach gesagt mit Präteritumfrequenz der Gruppe 3}

Die Korrelation der Präteritumfrequenz der Verben der Gruppe 3 und des Dialektalitätsgrads, der mit Hilfe der dialektalen/regionalen Varianten des Partizips gesagt ermittelt wurde, ist mit einem Wert von $0,556^{\star \star \star}(\mathrm{n}=88)$ ebenfalls mittelstark. Der Korrelationskoeffizient $\mathrm{R}^{2}$ beträgt dementsprechend 0,309.

Der Mittelwert der Standardnähe ist 71,2\%, der Mittelwert der Präteritumfrequenz 27,0\%. Die Standardabweichung ist bei der Standardnähe mit 39,2\% sehr hoch, bei der Präteritumfrequenz mit 26,1\% mittelhoch. Bei der Standardnähe gibt es also sehr viele Extremwerte, bei der Präteritumfrequenz verteilen sich die Werte auf ein breites Spektrum. 
Die nachfolgende Abbildung 11 verdeutlicht diese höchstsignifikante, mittelstarke Korrelation. Auf der y-Achse liegen die prozentualen Anteile an Präteritalverwendungen gegenüber den Perfektverwendungen der Verben aus Gruppe 3, auf der $\mathrm{x}$-Achse die relativen Werte der standardnahen Varianten des Partizips gesagt. Es zeigt sich ein linear positiver Zusammenhang zwischen der Präteritumfrequenz und der Standardnähe.

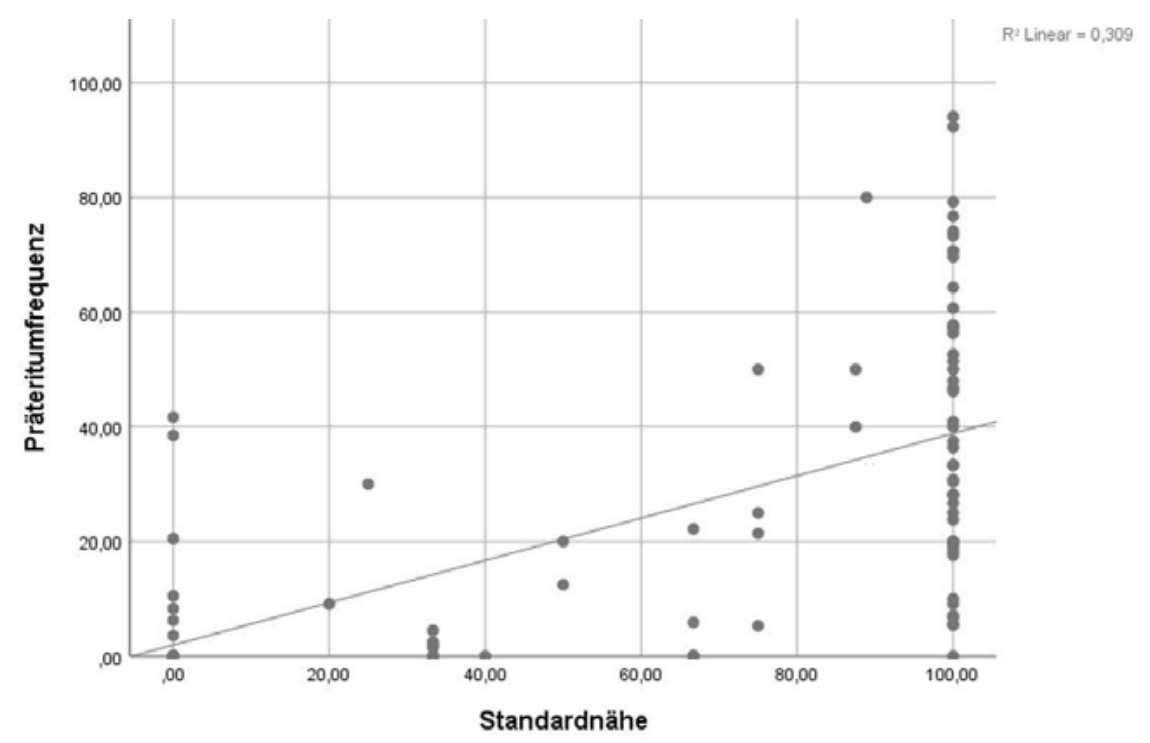

Abb. 11: Einfache Streuung mit Anpassungslinie für den Zusammenhang zwischen Präteritumfrequenz der Gruppe 3 und Standardnähe nach gesagt

\section{Zusammenfassung}

Zwischen der Präteritumfrequenz der Verben aus Gruppe 3 und der Standardnähe der Varianten von gehabt/gewesen und gesagt gibt es jeweils eine mittelstarke Korrelation. Standardnähere Sprecher verwenden also tendenziell mehr Präteritalformen der Verben aus Gruppe 3 als standardfernere Sprecher. Während die im Präteritum hochfrequenten Verben sein und sollen von standardnäheren und standardferneren Sprechern etwa gleich oft verwendet werden, ist bei den Verben aussehen, gehen, haben, heißen, kommen, liegen, müssen, stehen und wissen ein Unterschied in der Frequenz zu sehen, der mit der Standardnähe des jeweiligen Sprechers korreliert. Dieses Ergebnis lässt den Schluss zu, dass einige Verben (sein und sollen) vollkommen ins Alemannische Deutschlands integriert wurden und deshalb von allen Sprachschichten verwendet werden und dass 
andere Verben weniger stark integriert sind, weshalb diese von standardnäheren Sprechern häufiger verwendet werden als von standardferneren Sprechern.

\subsubsection{Das Präteritum als Entlehnung aus dem Standard?}

In diesem Kapitel gehe ich der Frage nach, ob die Präteritalformen aus dem Standard entlehnt wurden oder ob der Präteritumschwund im Alemannischen anders als bisher angenommen nicht vollständig abgeschlossen war. Ich untersuche hierzu, ob die Präteritum-Belege Standard- oder Dialektformen sind. Sollten die Präteritum-Belege standardnah sein, spräche dies für eine Entlehnung aus dem Standard. Da das Flexionssuffix $\{$ st $\}$ der 2. Person Singular des Präsens im Alemannischen palatalisiert wird, werte ich für diese Analyse die Belege der 2. Person Singular aller Verben des Präteritums aus. Zusätzlich untersuche ich eine Stichprobe aus 848 Belegen (708 aus REDI+FLARS (dt.) und 140 aus dem SSA) der Verben finden, geben, gehen, heißen, kommen, sein, stehen und wissen.

Für die Belege der 2. Person Singular zeigt sich, dass im SSA das Flexionssuffix $\{\mathrm{st}$ \} bei allen zehn Belegen palatalisiert wurde, in REDI+FLARS (dt.) wurde das Flexionssuffix bei 57 der 60 Belege palatalisiert. Die Auswertung der Stichprobe aus 848 Belegen zeigt außerdem in beiden Korpora bei nahezu allen Präteritalformen Lenisierungen oder Tilgungen. Von Lenisierungen ist vor allem das Phonem [t] zur Präteritalbildung bei den Modalverben und den irregulären Verben (z. B. denken und haben) betroffen. Beispiel (49) illustriert dies.

(49) Bötzingen (REDI+FLARS (dt.))

01 GP1_1112: früher hadde_ma noch viel KOHle,

Der Sprecher GP1_1112 verwendet hier bei der Präteritalform „hadde“ (Z. 01) den stimmhaften Plosiv [d] anstelle des stimmlosen Plosivs [t] des Standarddeutschen. Die Präteritalform der 3. Person Singular ist also an das Alemannische assimiliert.

Getilgt werden hauptsächlich die auslautenden Phoneme [ə] und [n]. Dies betrifft beispielsweise beim Modalverb sollen die 1. und 3. Person Singular und Plural (ich sollte $\rightarrow$ ich sollt; er sollte $\rightarrow$ er sollt; wir sollten $\rightarrow$ wir sollte und sie sollten $\rightarrow$ sie sollte) und beim Verb heißen die 1. und 3. Person Plural (wir hießen $\rightarrow$ wir hieße und sie hießen $\rightarrow$ sie hieße). Das folgende Beispiel (50) zeigt eine solche $n$-Tilgung am Wortende. 
(50) Breisach (REDI+FLARS (dt.))

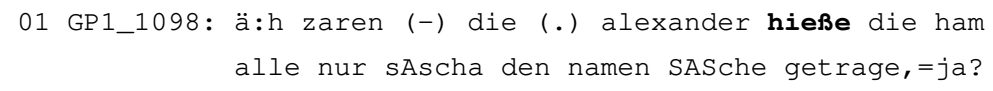

Das auslautende [n] der Präteritalform des standarddeutschen hießen ist hier wie bei den meisten Belegen der 3. Person Plural getilgt. Genauso wie bei Lenisierungen sind auch Tilgungen im SSA und in REDI+FLARS (dt.) gleichermaßen häufig zu beobachten.

In wenigen Fällen findet sich für die 3. Person Plural anstelle der [n]-Tilgung die standardfernere Variante des Flexionsmorphems \{-et\}. Die Beispiele (51) und (52) demonstrieren die Verwendungen dieser Variante. In Beispiel (51) erzählt die Sprecherin GP1_1313, dass sie in der Volksschule keine Fremdsprache gelernt hätte und dass ein oder zwei Schüler aufs Gymnasium gingen.

(51) Au am Rhein (REDI+FLARS (dt.))

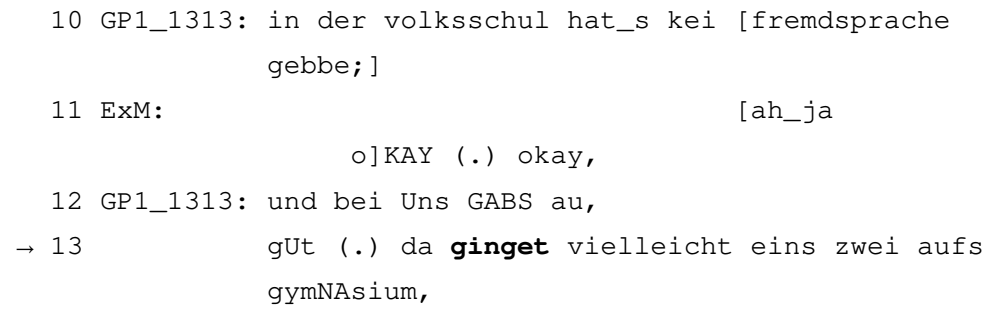

GP1_1313 verwendet bei der Präteritalform „ginget“ (Z. 13) das standardferne Flexionsmorphem $\{$ et $\}$. Auch im folgenden Beispiel (52) äußert eine Sprecherin dieses Morphem beim Verb „kAmet“ in Zeile 02.

(52) Bötzingen (REDI+FLARS (dt.))

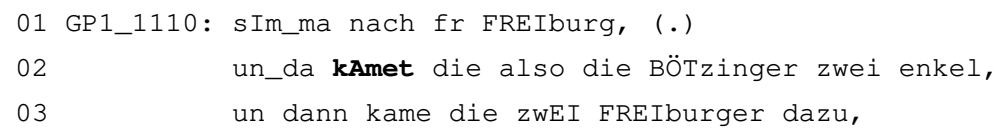

Bemerkenswert ist hierbei, dass GP1_1110 in Zeile 03 für die Präteritalform „kame“ die standardnähere Variante mit getilgtem [n] wählt.

Insgesamt sind in der Stichprobe aus REDI+FLARS (dt.) vier der 67 Belege der 3. Person Plural mit der Endung \{et\} realisiert. Das entspricht 6,0\%. Diese vier Belege entfallen auf die beiden Sprecherinnen GP1_1313 und GP1_1110. Im SSA ist der Anteil dieser standardferneren Variante mit 16,7\% etwas höher als in 
REDI+FLARS (dt.). Aufgrund der geringen Belegzahl von zwei Belegen mit \{et\} und zehn Belegen mit getilgtem [n] ist zwar unklar, ob die Anzahl der standardfernen Varianten tatsächlich abgenommen hat, allerdings würde sich eine solche Annäherung an den Standard mit den Ergebnissen aus Kapitel 3.1.7 decken.

Da nur zwei Sprecherinnen in der Stichprobe aus REDI+FLARS (dt.) das Flexionsmorphem $\{$ et $\}$ verwenden, lohnt eine nähere Betrachtung dieser Gewährspersonen. Ich vergleiche dabei jeweils das Verhältnis von $\{$ et $\}$ und $\{e\}$ als Flexionsendung der 3. Person Plural im Präteritum mit dem Präsens. Die Tabellen 22 und 23 illustrieren diese Verteilungen.

Tab. 22: Verteilung der Flexionsmorpheme $\{$ et $\}$ und $\{$ e $\}$ im Präteritum und Präsens bei GP1_1313

\begin{tabular}{lllllll}
\hline & \{et\} & \multicolumn{3}{c}{$\mathbf{e}$} & \multicolumn{3}{c}{ Gesamt } \\
\hline Präteritum & 2 & $(25,0 \%)$ & 6 & $(75,0 \%)$ & 8 & $(100 \%)$ \\
Präsens & 14 & $(87,5 \%)$ & 2 & $(12,5 \%)$ & 16 & $(100 \%)$ \\
\hline
\end{tabular}

Tab. 23: Verteilung der Flexionsmorpheme $\{e t\}$ und $\{e\}$ im Präteritum und Präsens bei GP1_1110

\begin{tabular}{lllllll}
\hline & \{et\} & \multicolumn{3}{c}{ \{e\} } & \multicolumn{3}{c}{ Gesamt } \\
\hline Präteritum & 2 & $(16,7 \%)$ & 10 & $(83,3 \%)$ & 12 & $(100 \%)$ \\
Präsens & 5 & $(50,0 \%)$ & 5 & $(50,0 \%)$ & 10 & $(100 \%)$ \\
\hline
\end{tabular}

Es zeigt sich, dass beide Sprecherinnen im Präteritum die Flexionsendung der 3. Person Plural \{et\} mit 25,0\% bzw. 16,7\% deutlich seltener verwenden als das Flexionsmorphem $\{\mathrm{e}\}$ mit 75,0\% bzw. 83,3\%. Im Präsens dagegen wird die standardfernere Variante mit 87,5\% bzw. 50,0\% deutlich häufiger gebraucht als im Präteritum.

Es lässt sich also festhalten, dass alle regiolektalen Merkmale des Alemannischen auch bei den Präterita angewendet werden. Das Flexionsmorphem \{et\}, das als standardferner $\mathrm{zu}$ betrachten ist als beispielsweise $n$-Tilgungen, findet dagegen kaum Verwendung. Das deutet darauf hin, dass es sich bei den Präteritum-Belegen um Entlehnungen aus dem Standard handelt, die an den Dialekt assimiliert wurden. Für die Entlehnung aus dem Standard spricht außerdem die Tatsache, dass es keine dialektspezifischen Formen des Präteritums gibt, wie die in manchen Ortsgrammatiken erfasste alte Form was des Indikativ Präteritum von sein (vgl. Fischer 1920: 1330 und Hufnagl 1967: 132). Die Präteritum-Belege 
im SSA und in REDI+FLARS (dt.) weisen also lediglich regiolektale, aber keine basisdialektalen Merkmale auf. Dagegen sind die Partizipien gehabt, gesagt, gewesen zur Perfektbildung im SSA in 97,4\%, 79,0\% bzw. 95,6\% und in REDI+FLARS (dt.) in 64,1\%, 21,8\% bzw. 66,3\% standardfern (vgl. Kap. 3.1.7).

\subsubsection{Zusammenfassung}

Ziel dieses Kapitels war es, die Entwicklung des Präteritums von 1974 bis 2013 zu beschreiben. Den Ausgangspunkt der Untersuchung bildete die Beobachtung, dass es ein frequentes Präteritum in meinen Daten gibt. Dabei zeigte sich, dass das Präteritum spätestens seit Mitte der 1970er Jahre im Alemannischen Deutschlands an Häufigkeit zunimmt.

In der Forschungsliteratur herrscht größtenteils die Meinung vor, eine Verwendung des Präteritums diene im Süddeutschen Raum dazu, vornehm und formell (vgl. Widén 1970: 283) oder besonders standardsprachlich und schriftdeutsch zu klingen (vgl. Fischer 2018: 213). Die Wirkung als formell oder schriftdeutsch entsteht durch das Code-Switching vom Dialekt zum Standard, mit dem häufig eine Verwendung des Präteritums einhergeht. Aus diesem Grund habe ich Äußerungen von der eigentlichen Analyse ausgeschlossen, die neben einer möglicherweise standardnahen Präteritalform verschiedene standardsprachliche Merkmale aufweisen und daher nicht Teil des eigentlichen Dialekts sind, sondern einen Wechsel vom Dialekt zum Standard darstellen.

Präteritalverwendungen finden sich aber nicht nur in standardnahen, sondern auch in dialektalen Sprechweisen. Dabei ist anzunehmen, dass das Präteritum aus dem Standard entlehnt und an das Badischalemannische phonologisch assimiliert wurde. Dafür spricht, dass fast alle Präteritalformen regiolektale Merkmale wie Palatalisierung, Tilgung und Lenisierung aufweisen, aber im Gegensatz zu den Partizipien keine dialektspezifischen Formen existieren. Dieses Ergebnis passt zu der Beobachtung von Müller-Wehingen (1930: 51), der im Saargau „ein Eindringen schriftsprachlicher Elemente“ in Form eines Präteritumgebrauchs der jüngeren Generation feststellt.

Obwohl das Präteritum durch den Kontakt mit dem Standard in das Badischalemannische wiedereingedrungen ist, zeigte sich beim Vergleich des Dialektalitätsgrads mit der Präteritumfrequenz der Sprecher nur eine schwache bis mittlere Korrelation. Während standardnähere Sprecher die Verben sein und sollen nur etwas häufiger im Präteritum verwenden als standardfernere Sprecher, gibt es zwischen dem Dialektalitätsgrad und der Präteritumfrequenz der Verben aussehen, gehen, haben, heißen, kommen, liegen, müssen, stehen und wissen eine 
mittelstarke Korrelation. Das heißt, die Verben sein und sollen sind vollständig, die restlichen Verben weitestgehend ins Badischalemannische Deutschlands integriert. Aufgrund dieser Durchdringung aller Sprachschichten und der Assimilation an den Regiolekt lässt sich das Präteritum zweifellos als Teil des Dialekts auffassen.

Ich konnte beim Vergleich des älteren Korpus SSA (1974-1985) mit dem jüngeren Korpus REDI+FLARS (dt.) (2007-2013) eine massive Zunahme der arealen Verteilung, der Tokenfrequenz und der Verbtypes, die Präteritum bilden, feststellen. Auch der Apparent-Time-Vergleich der älteren mit der jüngeren Sprechergruppe in REDI+FLARS (dt.) zeigte bei den meisten Verben einen deutlichen Anstieg der Präteritumfrequenz. Der Sprachwandel, der durch den Real-TimeVergleich der Korpora SSA und REDI+FLARS (dt.) ermittelt werden konnte, scheint sich auch beim Apparent-Time-Vergleich der älteren mit der jüngeren Sprechergruppe in REDI+FLARS (dt.) fortzusetzen.

Mit Ausnahme der Verben machen und mögen war zu beobachten, dass bei allen untersuchten Verben die areale Ausbreitung und die Tokenfrequenz der Präteritalformen zwischen dem SSA und REDI+FLARS (dt.) stark zugenommen haben, sodass in REDI+FLARS (dt.) in jedem Untersuchungsort Präteritalformen vorkommen. Dabei haben die Verben, die im SSA die größte areale Ausbreitung des Präteritums haben, auch in REDI+FLARS (dt.) die größte areale Ausbreitung des Präteritums. Für die Verben mit der geringsten Ausbreitung ist es umgekehrt.

Bei den Verben geben, kommen, werden, dürfen, können und müssen sind im SSA in fast allen Orten nur Perfektformen zu finden; in REDI+FLARS (dt.) ist das Perfekt-Präteritum-Verhältnis (beinahe) ausgeglichen. Bei den Verben haben und gehen kommt das Präteritum nur vereinzelt im Untersuchungsgebiet des SSA vor. In REDI+FLARS (dt.) ist der Anteil an Perfekt zwar immer noch höher; aber der des Präteritums hat deutlich zugenommen. Die Verben sollen und sein weisen bereits im SSA ein in etwa ausgeglichenes Verhältnis der beiden Tempora auf. In REDI+FLARS (dt.) stellt bei diesen Verben nun das Präteritum die überwiegende Mehrheit an Belegen dar. Besonders auffällig ist die Verteilung beim Modalverb wollen. Bei diesem ist im SSA der Anteil an Perfekt deutlich größer als der des Präteritums; in REDI+FLARS (dt.) dagegen überwiegt das Präteritum deutlich.

Ein Vergleich der Einwohnerzahl der Orte mit der Anzahl an verwendeten Verbtypes zeigte, dass keine signifikante Korrelation zwischen der Größe des Ortes und der Präteritumverwendung besteht. Die Präteritumverwendung beschränkt sich also, anders als von Rowley (1983: 165) vermutet, nicht auf größere Orte, sondern umfasst das gesamte Untersuchungsgebiet.

Auch die relative Frequenz des Präteritums (verglichen mit der des Perfekts) aller Verben, die im Gesamtkorpus mindestens zweimal gebraucht werden, hat 
vom SSA zu REDI+FLARS (dt.) zugenommen. Die relative Frequenz der Präteritalformen des Verbs sagen ist auch in REDI+FLARS (dt.) extrem gering. Die restlichen 22 Verben, die in REDI+FLARS (dt.) im Präteritum vorkommen, sind dagegen mit relativen Frequenzen des Präteritums zwischen 7,9\% und 90,3\% zweifellos in Gebrauch.

Bei der Frage, welche Verben das Präteritum bilden, stellte sich heraus, dass 16 der 22 Verben in REDI+FLARS (dt.), die ein Präteritum aufweisen, Zustandsverben sind. Von den restlichen sechs besitzen fünf Verben eine stative Teilbedeutung und werden hauptsächlich oder ausschließlich in dieser Teilbedeutung im Präteritum gebraucht. Das sind Bedeutungen, die von der Grundbedeutung verschieden sind und die aktionsartlich als State zu beurteilen sind. Fischer stellt einen stärkeren Präteritumerhalt bei irregulären und starken Verben fest: „Es sind häufige Verben, die zum Grundwortschatz gehören und von denen viele eine imperfektive Verbsemantik haben“ (Fischer 2018: 390; vgl. auch Harnisch 1997: 120-123 sowie Trost 2019). Die wieder in den Dialekt eingedrungenen Präteritalformen betreffen also genau die Verben, die besonders resistent gegenüber dem Präteritumschwund waren. Diese lassen sich daher als besonders präteritumaffin bezeichnen. Ich werde in Kapitel 3.2.4 auf die Gründe eingehen, warum gerade diese Verben beziehungsweise deren Teilbedeutungen für das Präteritum empfänglich sind.

Fischer (2018) stellt zudem eine Abbauhierachie des Präteritumschwunds nach Verbklassen vor, nach der das Verb sein besonders resistent ist, gefolgt von Modal-/Hilfsverben, irregulären/starken Verben und schwachen Verben. Diese Abbauhierarchie spiegelt sich nur grob im Wiedereindringen des Präteritums ins Badischalemannische wider. Tatsächlich ist das Verb sein mit 42,8\% und 90,3\% in REDI+FLARS (dt.) in beiden Korpora sehr häufig im Präteritum und über das gesamte Untersuchungsgebiet etwa gleich verteilt. Bei den Modal- und Hilfs-verben zeigt sich ein heterogenes Bild. Während das Modalverb mögen in keinem der beiden Korpora im Präteritum zu finden ist (dies liegt vermutlich auch an der grundsätzlich extrem niedrigen Frequenz dieses Verbs in einem Vergangenheitstempus), zählen die restlichen Modalverben zu den Verben mit der höchsten relativen Frequenz an Präteritum. Diese unterscheidet sich zwischen den Modalverben allerdings deutlich. Das Modalverb sollen weist mit 44,1\% und 87,8\% in den beiden Korpora nach dem Kopulaverb sein die relativ gesehen meisten Präteritalformen auf. Bei dürfen, können, müssen und wollen ist die relative Präteritumfrequenz dagegen mit Werten zwischen 0,9\% und 6,6\% im SSA und mit Werten zwischen $37,7 \%$ und $74,7 \%$ in REDI+FLARS (dt.) (deutlich) geringer. Auch beim Hilfsverb werden bestätigt sich die Beobachtung Fischers. Dieses ist mit 12,3\% bzw. 49,7\% ähnlich häufig im Präteritum zu finden wie die Modalverben. 
Die Präteritalformen der Hilfsverben haben und sein wurden in diesem Kapitel nicht betrachtet. In Kapitel 4.2.4 gehe ich auf diese beiden Hilfsverben zur Plusquamperfektbildung näher ein und zeige, dass das Hilfsverb sein mit 62 Belegen im SSA und 28 Belegen in REDI+FLARS (dt.) und das Hilfsverb haben mit zwei bzw. 36 Belegen extrem selten vorkommen. Die irregulären und starken Verben sind mit Frequenzen von $0 \%$ bis $7,3 \%$ im SSA und von $7,9 \%$ bis $57,5 \%$ in REDI+FLARS (dt.) durchschnittlich seltener als die Modal- und Hilfsverben. Allerdings sind einzelne starke Verben (beispielsweise geben in REDI+FLARS (dt.) mit 57,5\%) deutlich frequenter als einzelne Modal- oder Hilfsverben (beispielsweise müssen mit 37,7\%). Fischers Beobachtung ist also grundsätzlich zu bestätigen, wobei einige Ausnahmen zu erkennen sind.

Es konnte eine starke Zunahme des Präteritums in arealer Ausbreitung, Verbtypes und Tokenfrequenz beobachtet werden. Während es im SSA nur vereinzelt vorkommt, ist das Präteritum in den jüngeren Daten fester Bestandteil des Badischalemannischen. Obwohl eine leichte Korrelation zwischen Dialektalitätsgrad und Präteritumverwendung festgestellt werden konnte, umfasst das Präteritum alle Sprachschichten sowie alle Untersuchungsorte. Das folgende Kapitel behandelt die Frage, welche Auswirkungen sich auf das Tempusgefüge bei der Koexistenz zweier Tempora der einfachen Vergangenheit ergeben.

\subsection{Perfekt \& Präteritum im heutigen Alemannischen in Baden}

In Kapitel 3.1 konnte ich aufzeigen, dass das Präteritum seit der Mitte des 20. Jahrhunderts im Alemannischen Deutschlands hinsichtlich arealer Ausbreitung und Häufigkeit im Vergleich mit der Perfektform sowie der Anzahl an Verben, bei denen eine Präteritumform nachweisbar war, stark zugenommen hat. Dabei konnte ich für 22 Verben ein frequentes Präteritum im Korpus REDI+FLARS (dt.) nachweisen. In diesem Kapitel möchte ich nun Perfekt und Präteritum als koexistierende Tempora der einfachen Vergangenheit in REDI+FLARS (dt.) miteinander vergleichen. Da ich in diesem Kapitel keinen diachronen Vergleich anstelle, ist eine Unterscheidung in eine jüngere und eine ältere Sprechergruppe nicht zielführend. Ich betrachte lediglich die 22 Verben, die ein Präteritum bilden, da Perfekt und Präteritum auch nur bei diesen miteinander konkurrieren können. Dabei stellt sich die Frage, ob Perfekt und Präteritum - wie auch im Standarddeutschen - zwei verschiedene Funktionen und Verwendungsweisen haben. Zunächst gebe ich daher einen Überblick über die in der Forschungsliteratur beschriebenen Funktionen der beiden Tempora im Standarddeutschen und ergänze diese mit eigenen Überlegungen hinsichtlich der Verwendungskontexte. 


\subsubsection{Funktionen von Perfekt und Präteritum im Standarddeutschen}

Die meisten Autoren sehen einen semantischen Unterschied zwischen Perfekt und Präteritum (Hennig 2000: 26). Gegensätzliche Meinungen finden sich vor allen Dingen in Arbeiten der DaF-Literatur. Jäger (2007: 29) bemerkt, dass im DaFBereich Perfekt und Präteritum als prinzipiell immer austauschbar dargestellt werden und sich unterschiedliche Verwendungen durch Textsorte und regionale Unterschiede ergäben. In den meisten anderen Arbeiten werden dem Perfekt im Gegensatz zum Präteritum verschiedene Bedeutungen zugeschrieben. Das Perfekt kann demnach auch auf gegenwärtige und zukünftige Ereignisse verweisen. Neben diesen Bedeutungsvarianten, die offensichtlich nicht mit dem Präteritum austauschbar sind, kann das Perfekt auch Vergangenheit ausdrücken. ${ }^{22}$ Inwieweit es in dieser Vergangenheitsbedeutung mit dem Präteritum ausgetauscht werden kann, ist allerdings umstritten.

Grundlegend für die Beschreibung des Perfekts ist bei den meisten Autoren die Annahme, das Perfekt sei ambig (vgl. u. a. Thieroff 1992: 165 und Hennig 2000: 26). Dabei gäbe es eine Variante, die sich mit dem Präteritum austauschen lasse (=nicht-perfektisches Perfekt), und eine Variante, die sich nicht mit dem Präteritum austauschen lasse (=perfektisches Perfekt) (Hennig 2000: 26). Hennig stellt die These auf, dass „nicht jedes Perfekt durch ein Präteritum, aber jedes Präteritum durch ein Perfekt ersetzt werden kann“ (Hennig 2000: 27, FN 47). Während dem Präteritum meistens keine spezifischen Funktionen abseits der Vergangenheitsbedeutung zugeschrieben werden, weisen die meisten Autoren dem perfektischen Perfekt eine spezifische Funktion zu, die es auch in der Vergangenheitsbedeutung vom Präteritum abgrenzt.

Einige Autoren sehen trotz unterschiedlicher Beschreibungsmuster als spezifische Funktion des Perfekts Gegenwartsbezug oder Resultativität an (vgl. u. a. Ehrich/Vater 1989: 107-110, Zifonun et. al. 1997: 1697-1702, Weinrich 2007: 227 und Duden 2016: 518). Leiss (1992: 156 und 278) und Gillmann (2016: 315) erkennen dagegen nur beim Perfekt mit Hilfsverb sein eine Tendenz zur Resultativität. Für eine Gegenwartsrelevanz, aber explizit gegen eine Resultativitätsbedeutung spricht sich vor allen Dingen Thieroff aus, da Sätze mit im Perfekt stehendem finiten Verb nicht oder nur schwer mit der adverbialen Angabe (immer) noch verbunden werden könnten (1992: 176). Thieroffs Idee ist dabei, dass für

22 Hierbei ist anzumerken, dass in bestimmten formelhaften Verwendungen auch das Präteritum auf Gegenwärtiges oder Zukünftiges referieren kann. Dabei handelt es sich jedoch um feste Konstruktionen, die nicht mit dem Perfekt gebildet werden können (vgl. dazu Sätze wie Wer bekam das Schnitzel? (siehe auch Rödel 2007: 61)). 
Resultativität notwendigerweise das beschriebene Ereignis und die erzielte Wirkung gleichzeitig Gültigkeit besitzen müssten (Thieroff 1992: 176).

In anderen Arbeiten findet sich häufig als spezifische Perfektbedeutung Abgeschlossenheit beziehungsweise Vollzug oder Vollendung (Brinkmann 1971: 339-340, Glinz 1971: 149 und Jung 1984: 200). Obwohl nur wenige Autoren mit den Begriffen perfektiv und imperfektiv (vgl. Eisenberg 1994: 121) sowie perfektiv und durativ (vgl. Heidolph et al. 1984: 508-509) von Aspekt im eigentlichen Sinn sprechen, lassen auch die Beschreibungen der anderen hier zitierten Autoren den Schluss zu, das Perfekt könne perfektiven Aspekt (=abgeschlossen) ausdrücken. Darüber hinaus schreiben einige Autoren dem Perfekt in einigen Kontexten Resultativität beziehungsweise Gegenwartsrelevanz und in anderen Abgeschlossenheit zu (Erben 1980: 94, Heidolph et. al. 1984: 514 und Welke 2005: 315-316). Einen Vorschlag zur Zusammenführung dieser beiden eigentlich unvereinbaren Positionen (Abgeschlossenheit vs. Resultativität/Nicht-Abgeschlossenheit) machen unter anderem Musan (1999: 36-37), Helbig/Buscha (2001: 135), Szczepaniak (2011: 133-134) und Fischer (2018: 192) mit der Überlegung, dass das Perfekt eine Handlung beschreibt, die abgeschlossen ist und deren Resultat fortbesteht. Diese Perfektverwendung lässt sich auch als gegenwartsbezogene Vergangenheit bezeichnen.

Ehrich/Vater (1989: 124) und später Zifonun et al. (1997: 1697-1702) erklären diesen Gegenwartsbezug des Perfekts mit Hilfe der Referenzzeit. Während beim Präteritum Referenz- und Ereigniszeit zusammenfallen, die beide zeitlich vor der Sprechzeit liegen, sind beim Perfekt Referenz- und Sprechzeit identisch, die zeitlich nach der Ereigniszeit lokalisiert sind (Ehrich/Vater 1989: 124). Beim Perfekt betrachtet der Sprecher also von der in der Gegenwart liegenden Referenzzeit aus ein Ereignis. Dadurch entstehe ein Gegenwartsbezug. Fischer bezeichnet diese Gegenwartslesart des Perfekts ausgehend von der Beschreibung Ehrich/Vaters (1989) als retrospektiven Aspekt (2018: 197) (vgl. Kap. 2.1.1). Dieser Aspekt entstehe, indem die Referenzzeit der Ereigniszeit nachgeordnet und zeitgleich zum Folgezustand einer Situation lokalisiert wird. Der Nachzustand diene als Bezugspunkt für die Referenzzeit, von der aus die Verbalsituation retrospektiv betrachtet werden könne (ebd.).

Unabhängig davon, ob die Gegenwartslesart des Perfekts nur durch die Anordnung der Reichenbach'schen Zeiten zu erklären ist oder als (retrospektiver) Aspekt zu verstehen ist, bedarf es einer empirischen Untersuchung des möglichen Gegenwartsbezugs des Perfekts. Ich werde daher in Kapitel 3.2.2 der Frage nachgehen, ob das Perfekt im Gegensatz zum Präteritum eine Gegenwartsrelevanz besitzen kann. 
In Kapitel 2.2.1 habe ich die These vorgestellt, dass Aspekt in den meisten Sprachen Ausdruck findet. Allerdings wird Aspekt nur in einigen wenigen Sprachen als grammatische Kategorie am Verb markiert. Da es im Deutschen und im Alemannischen keine grammatisch am Verb angezeigte Aspektopposition gibt (wie beispielsweise in den slawischen Sprachen), stellt sich die Frage, ob mittels der Tempusvariation Perfekt/Präteritum der perfektive und imperfektive Aspekt vermittelt wird. Um dies zu überprüfen, betrachte ich Äußerungen mit Perfekt als finitem Verb und Äußerungen mit Präteritum als finitem Verb und untersuche, ob eine Korrelation zwischen der Tempusverwendung und abgeschlossenen und unabgeschlossenen Situationen besteht. Sollten Äußerungen mit Perfekt als finitem Verb systematisch abgeschlossene Situationen beschreiben und Äußerungen mit Präteritum als finitem Verb unabgeschlossene Situationen, läge der Schluss nahe, dass es im Alemannischen eine grammatische Kategorie Aspekt gibt und die Aspektopposition perfektiv/imperfektiv mit Hilfe der Tempusvariation ausgedrückt wird (Kap. 3.2.3).

Daneben könnten auch die Aktionsarten Informationen signalisieren, die eigentlich vom Aspekt vermittelt werden (Situationen als abgeschlossen oder unabgeschlossen darzustellen). Daher vergleiche ich Perfekt und Präteritum hinsichtlich möglicher Affinitäten $\mathrm{zu}$ Aktionsarten. Dabei betrachte ich nur diejenigen Verben, die mehrere Aktionsarten haben können. Hierzu zählen die Verben finden, geben, gehen, heißen und kommen (Kap. 3.2.4).

Neben den möglichen semantischen Unterschieden zwischen Perfekt und Präteritum könnten sich auch aufgrund der verschiedenen morpho-syntaktischen Beschaffenheit der beiden Tempora Unterschiede in der Verwendung ergeben. Das Perfekt bildet im Gegensatz zum Präteritum eine Verbalklammer, d. h. die rechte Satzklammer ist bei Perfektkonstruktionen immer besetzt. Der Verbalklammer wird von vielen Autoren eine wichtige Funktion im Deutschen zugeschrieben (vgl. Sieberg 1984: 147, Ronneberger-Siebold 1991: 208-209, Leiss 1992: 278, Nübling et al. 2017: 117-127 und Fischer 2018: 355). Leiss (1992: 278) und Fischer (2018: 129) stellen fest, dass das Präteritum von Verben, die zur Klammerbildung neigen, (häufiger) erhalten ist (so z. B. Modalverben, vgl. Kap. 3.1), da bei diesen Verben die Verwendung des Perfekts keinen Vorteil bringt. Glinz betont, dass mit der Klammerbildung des Perfekts eine „größere Umständlichkeit“ (1971: 152) einhergeht. Das Präteritum ermögliche dagegen knappere und konzentriertere Aussagen (ebd.).

Es stellt sich nun die Frage, ob in Kontexten, in denen die rechte Satzklammer bei beiden Tempora besetzt ist, vermehrt Präteritum gebraucht wird. Ich untersuche deshalb, ob die Stellung des finiten Verbs einen Einfluss auf die Tempuswahl hat (Kap. 3.2.5). 
Zudem betrachte ich Passivkonstruktionen mit den Hilfsverben sein und werden und vergleiche die Anzahl der Perfekt- und Präteritumformen, mit denen das Passiv gebildet wird. Sollte die Klammerbildung tatsächlich ein entscheidender Vorteil des Perfekts gegenüber dem Präteritum sein, ist anzunehmen, dass (verglichen mit dem Perfekt) in Passivkonstruktionen häufiger Präteritum gebraucht wird als in Aktivkonstruktionen. Sieberg (1984: 95) weist für die Rheinische Umgangssprache nach, dass das Perfekt von Passiv- und Modalverbkonstruktionen vermieden und stattdessen das Präteritum gebraucht wird. Auch er nennt eine sprachökonomische Erklärung, also den Umstand, dass die Passiv- und Modalverbkonstruktionen des Präteritums „ein sprachliches Element weniger auf[weisen]“ (Kap. 3.2.6). Da das Perfekt im Gegensatz zum Präteritum aus zwei Verbteilen besteht, die bei Passivkonstruktionen und Verbletztsätzen mit in die rechte Satzklammer gesetzt werden, würden Perfektkonstruktionen in diesen Fällen zu womöglich unerwünschten Clusterbildungen führen. Abschließend fasse ich die Ergebnisse dieses Kapitels zusammen (Kap. 3.2.7).

\subsubsection{Gegenwartsrelevanz des Perfekts}

In diesem Kapitel untersuche ich, ob das Perfekt im Gegensatz zum Präteritum eine Gegenwartsrelevanz besitzen kann. Äußerungen haben Gegenwartsrelevanz, wenn sie Situationen beschreiben, die in der Vergangenheit abgeschlossen sind und sich auf die Sprechzeit beziehen. Dementsprechend werden fortdauernde Situationen, die zum Sprechzeitpunkt immer noch Gültigkeit besitzen, nicht als gegenwartsrelevant im obigen Sinne betrachtet, da sie in der Vergangenheit nicht abgeschlossen sind. Beispielsätze wie (53) stellen daher keinen Bezug zur Gegenwart her.

(53) Ich kam 1990 nach Berlin. Es war eine große Stadt. Alles war anonym.

Obwohl die Aussage, dass Berlin eine große Stadt ist, auch zum Sprechzeitpunkt Gültigkeit besitzt, drückt das Verb war hier keine Gegenwartsrelevanz aus.

Einen Spezialfall der Gegenwartsrelevanz stellt die resultative Lesart dar (vgl. Thieroff 1992: 172-177). Unter resultativer Lesart verstehe ich einen direkten Kausalzusammenhang zwischen der im Perfekt stehenden Aussage und einem Resultat in der Gegenwart, bei dem die Aussage im Perfekt die Ursache direkt impliziert. Wunderlich führt als Beispiel den Satz (54) für eine resultative Lesart an.

„Bettina ist eingeschlafen“ 
Dieser Satz impliziere direkt, dass Bettina nun schlafe. Der Satz (55) impliziert diese Folge dagegen nicht (Wunderlich 1970: 142).

(55) „Bettina schlief ein“

Untersucht wird eine Stichprobe aus 2187 Belegen, die sich aus etwa gleich vielen Präteritum- und Perfektformen zusammensetzt (1101 Präterita und 1086 Perfekte). Die Gegenwartsrelevanz lässt sich bei allen Situationen nachweisen, die beispielsweise durch gegenwartsbezogene Adverbien wie jetzt eine Referenz zur Sprechzeit herstellen. Situationen, die sich nur auf die recent past (vgl. Smith 2011) beziehen, aber keinen weiteren Bezug zur Gegenwart aufweisen, werte ich dagegen nicht als gegenwartsrelevant.

Die Beispiele (56) und (57) verdeutlichen gegenwartsrelevante Situationen mit dem Adverb jetzt.

(56) Breisach (REDI+FLARS (dt.))

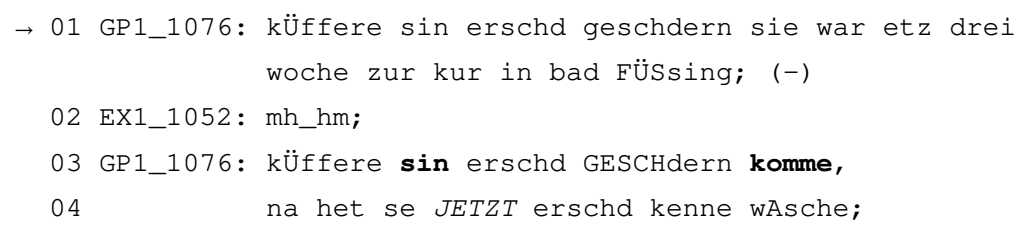

In diesem Beispiel erzählt die Gewährsperson GP1_1076 von einer Bekannten, die auf Kur war (Z. 01). Da deren Koffer erst gestern angekommen seien, habe sie jetzt erst waschen können (Z. 03-04). Die Äußerung in Zeile 01 ist nicht als gegenwartsrelevant zu sehen, da das Adverb „etz“ keinen Bezug zur Gegenwart herstellt. Stattdessen wird lediglich ein kürzlich zurückliegendes Ereignis beschrieben. Im Gegensatz dazu weist die Äußerung in Zeile 03 Gegenwartsrelevanz auf, da die Ankunft der Koffer kausal mit der Möglichkeit, die Wäsche dieser Koffer zu waschen, zusammenhängt.

Beispiel (57) ist ein Ausschnitt eines Gesprächsendes.

(57) Tiengen (REDI+FLARS (dt.))

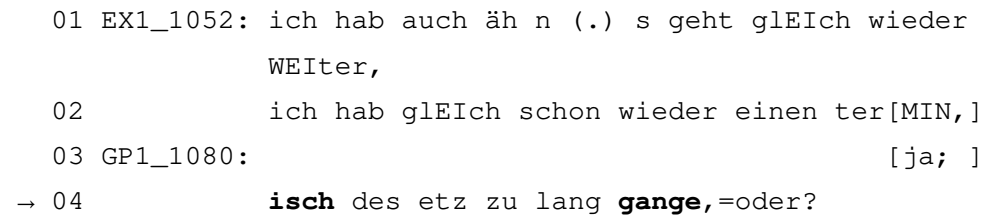




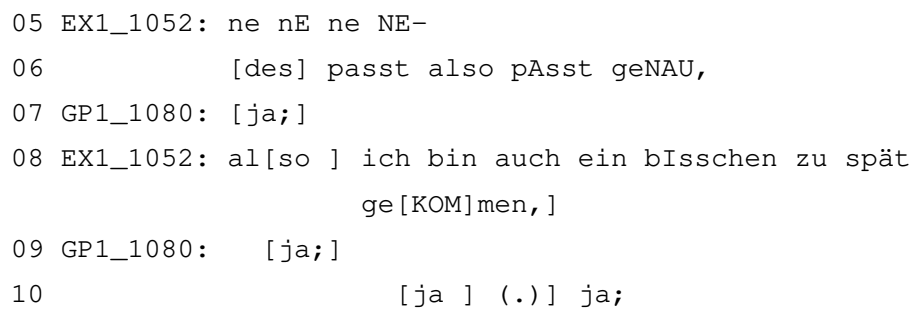

Der Interviewer erklärt hier, er habe gleich noch einen Termin (Z. 01-02). GP1_1080 fragt daraufhin, ob dieses Gespräch zu lange gegangen sei (Z. 04). Der Interviewer verneint dies in Zeile 05 und erklärt, dass es zeitlich in Ordnung sei (Z. 06). Außerdem sei er zu spät gekommen (Z. 08). Die Frage der Gewährsperson GP1_1080 stellt einen Bezug zur gegenwärtigen Situation her dar, da erfragt wird, ob die Dauer des Gesprächs die Ursache für die Verspätung sei.

Doch auch ohne ein auf die Gegenwart verweisendes Adverb kann ein Bezug zur Sprechzeit hergestellt werden. In einigen Fällen ergibt sich die Gegenwartsrelevanz aus dem Kontext. In Beispiel (58) antwortet die Sprecherin GP1_1064 auf die Frage, ob in ihrem Ort genauso starker Dialekt gesprochen wird wie im Nachbarort.

(58) Bombach (REDI+FLARS (dt.))

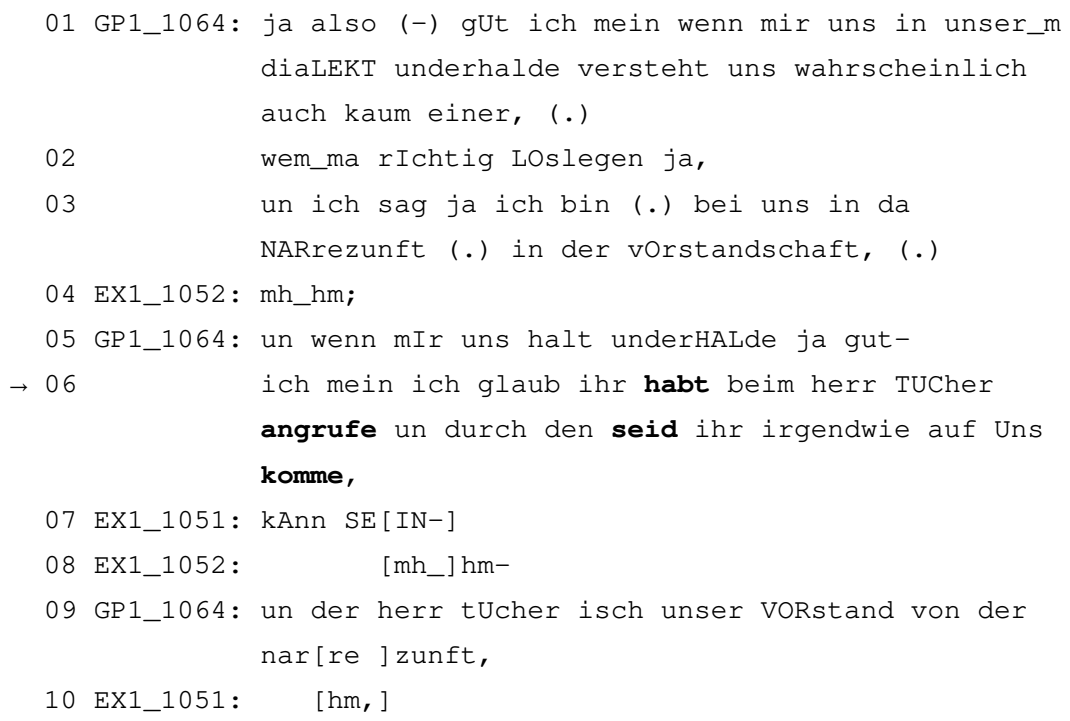


GP1_1064 beantwortet in den Zeilen 01 und 02 die Frage, wie sehr in ihrer Gegend Dialekt gesprochen wird, und erklärt, dass sie wahrscheinlich kaum verstanden werden. Sie expandiert ihre Antwort ab Zeile 03, indem sie auf die Narrenzunft verweist, bei der sie Mitglied ist. Ihre Aussage in Zeile 05 unterbricht sie vor der Beurteilung, wie stark dort Dialekt gesprochen wird, für eine Frage an die Interviewer (,ich mein ich glaub ihr habt beim herr TUCher angrufe un durch den seid ihr irgendwie auf Uns komme,“). Dass es sich dabei um eine Frage handelt, wird dadurch deutlich, dass die Interviewer EX1_1051 und EX1_1052 dem zustimmen. Nach der Beantwortung der Frage führt die Gewährsperson ihre Erzählung fort. Ihre Äußerung in Zeile 06 hat Gegenwartsrelevanz, da sie direkt auf die momentane Situation Bezug nimmt. Sie erfragt, ob es eine Verbindung gibt zwischen dem Anruf bei Herrn Tucher und der Tatsache, dass EX1_1051 und EX1_1052 sie nun interviewen.

Von den 2187 untersuchten Belegen wiesen nur 47 Gegenwartsrelevanz auf. Bei diesen handelt es sich ausschließlich um Perfekt-Belege. Die extrem geringe Zahl von 47 (4,3\% der in dieser Stichprobe untersuchten Perfekte) ist wohl durch die Art der Daten zu erklären. Da in den Interviews durch Erzählimpulse Erzählungen evoziert werden sollten, kommen die meisten Vergangenheitstempora in Kontexten vor, die keinen Bezug zur Gegenwart haben können. Dementsprechend kann keine Auskunft darüber gegeben werden, wie das Verhältnis von perfektischem zu nicht-perfektischem Perfekt tatsächlich ist. Da alle 47 Fälle mit Gegenwartsbezug im Perfekt realisiert sind, kann man trotz der vergleichsweise geringen Belegzahl davon ausgehen, dass das Perfekt eine Lesart besitzt, die das Präteritum nicht hat. Damit lassen sich die in Kapitel 3.2.1 dargelegten Überlegungen aus der Forschungsliteratur bestätigen und das Perfekt in ein perfektisches (=Gegenwartsbezug) und in ein nicht-perfektisches (=kein Gegenwartsbezug) unterteilt werden. Da nur die zweite Lesart mit dem Präteritum konkurriert, betrachte ich im Folgenden nur noch die 2140 Fälle, in denen kein Gegenwartsbezug vorhanden ist.

\subsubsection{Haben Perfekt und Präteritum Aspektfunktion?}

\section{Indizien für die Zuordnung zu abgeschlossenen und unabgeschlossenen Situationen}

Ich untersuche in diesem Kapitel, ob eine Korrelation zwischen Äußerungen mit Perfekt beziehungsweise Präteritum und abgeschlossenen beziehungsweise unabgeschlossenen Situationen besteht. Die Korrelation eines Tempus mit (Un-)Abgeschlossenheit würde darauf hindeuten, dass mit Hilfe der Tempusvariation der 
perfektive und imperfektive Aspekt ausgedrückt werden (vgl. Kap. 2.2). Die wichtigsten Indizien für die Zuordnung zu unabgeschlossenen Situationen sind neben der Einordnung ins Inzidenzschema (vgl. Kap. 2.2.2) temporale Adverbialangaben, die Regelmäßigkeit, Allgemeingültigkeit oder eine nicht abgegrenzte Zeitdauer ausdrücken. Zu diesen zählen die dialektalen Entsprechungen von Adverbien wie immer, ständig, zu dieser Zeit, grundsätzlich und täglich.

Demgegenüber weisen Temporalangaben, die ein einmaliges Ereignis oder einen festen Zeitraum beschreiben, auf Abgeschlossenheit hin. Dies sind dialektale Entsprechungen von Adverbien wie einmal oder Jahreszahlen wie (19)54. Die Beispiele (59-61) zeigen den Zusammenhang zwischen Temporalangabe und (Un-)Abgeschlossenheit einer Situation. Dabei weist die Angabe in Beispiel (59) auf ein regelmäßiges, nicht eingegrenztes Ereignis und damit auf eine unabgeschlossene Situation hin. Die Beispiele (60-61) zeigen abgeschlossene Situationen, da sie ein einmaliges Ereignis beschreiben.

(59) Tiengen (REDI+FLARS (dt.))

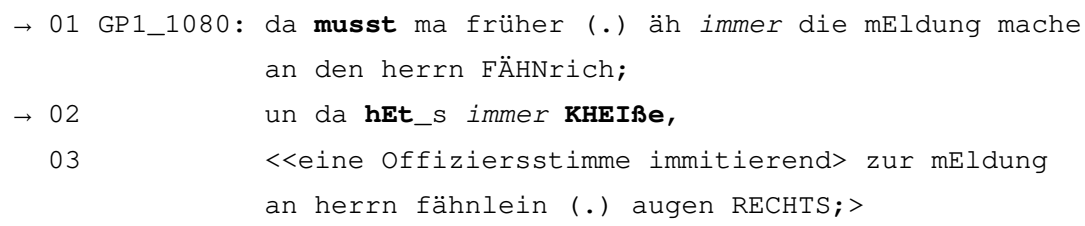

(60) Bombach (REDI+FLARS (dt.))

$\rightarrow 01$ GP1_1064: weil (.) er isch au eimal ned PÜNKTlich hEImkomme, gAb_s halt au ÄRger;

(61) Waldkirch (REDI+FLARS (dt.))

$\rightarrow 01$ GP1_1053: vierafünfzig bin_ich dann aus da SCHUL komma;

Während in diesen Fällen die (Un-)Abgeschlossenheit eindeutig durch Temporaladverbien ersichtlich wird, ist in der Mehrzahl der Fälle kein Temporaladverb vorhanden. Dort wird teilweise durch den Kontext klar, ob die Situation abgeschlossen oder unabgeschlossen ist. Lässt sich allerdings weder durch den Kontext, Weltwissen oder eindeutige Indizien wie Temporaladverbien die (Un-)Abgeschlossenheit bestimmen, gibt die Aktionsart des Verbs Auskunft darüber, ob die Situation abgeschlossen oder unabgeschlossen ist. Hier zeigt sich die Schnittstelle zwischen Aktionsart und (Un-)Abgeschlossenheit einer Situation. Während 
die durativen und nicht-grenzbezogenen (=atelischen) Aktionsarten Activity und State ohne weiteren Kontext auf unabgeschlossene Situationen hindeuten, signalisieren die Aktionsarten Accomplishment, Achievement und Semelfactive abgeschlossene Situationen. Die folgenden Beispiele (62-66) verdeutlichen dies.

(62) Todtmoos (REDI+FLARS (dt.)) - Activity

$\rightarrow 01$ GP1_1256: un auch in ENGland hat_se Underricht gegebbe,

In diesem Beispiel (62) erzählt die Sprecherin GP1_1256, dass ihre Tochter in England unterrichtet habe. Das Funktionsverbgefüge Unterricht geben ist hier semantisch mit dem Verb unterrichten gleichzusetzen, das eine zeitlich ausgedehnte (=durative) und nicht-grenzbezogene (=atelische) Handlung beschreibt. Da abgesehen von der lokalen Angabe „in ENGland“ (Z. 01) Indizien fehlen, die auf Abgeschlossenheit oder Unabgeschlossenheit hindeuten, ist diese Äußerung aufgrund der Aktionsart des Verbs als unabgeschlossen zu verstehen.

(63) Todtnauberg (REDI+FLARS (dt.)) - State

$\rightarrow 01$ GP1_1243: dIE war auf_da reALschul;

GP1_1243 berichtet hier, seine Tochter sei auf der Realschule gewesen (Z. 01). Da keine Festlegung auf einen begrenzten Zeitraum oder ein einmaliges Ereignis erfolgt, wird die unabgeschlossene Situation ausschließlich durch die Aktionsart des stativen Verbs „war“ deutlich.

(64) Bötzingen (REDI+FLARS (dt.)) - Accomplishment

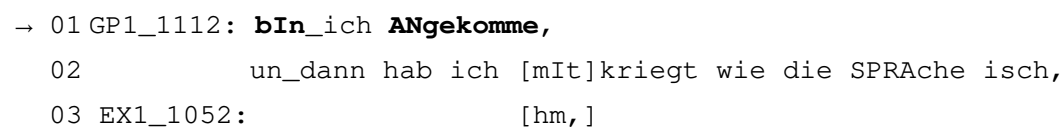

GP1_1112 erzählt hier von einem Urlaub in den USA. Er sei angekommen (Z. 01) und hätte dann erfahren, wie sich die englische Sprache anhöre (Z. 02). Es sind keine Indizien vorhanden, die auf eine Regelmäßigkeit oder eine unbestimmte Zeitdauer verweisen. Dementsprechend liefert ausschließlich die Aktionsart des Verbs ankommen Hinweise auf die (Un-)Abgeschlossenheit dieser Äußerung. Da es sich um ein Accomplishment handelt, das Durativität, aber auch Telizität vermittelt, ist die Äußerung als abgeschlossen zu betrachten. 
(65) Auenheim (REDI+FLARS (dt.)) - Achievement

$\rightarrow 01$ GP1_1324: wie ich äh (.) des (.) ich hab einfach des wort

(.) ned GFuNde oder au gAr ned (.) ja,

Die Gewährsperson GP1_1324 erzählt hier von Wortfindungsproblemen. Die Aktionsart des Verbs finden ist hier ein Achievement. Es ist daher telisch und nichtdurativ und kann nicht als Rahmenhandlung dienen, in die weitere Situationen inzidiert werden (vgl. das Inzidenzschema in Kap. 2.2.2). Indizien, die auf eine Regelmäßigkeit des eigentlich einmaligen Vorgangs hindeuten, fehlen hier. ${ }^{23}$ Aus diesem Grund bestimmt alleine die Aktionsart des Verbs die abgeschlossene Perspektive der Gesamtäußerung.

(66) Tiengen (REDI+FLARS (dt.)) - Semelfactive

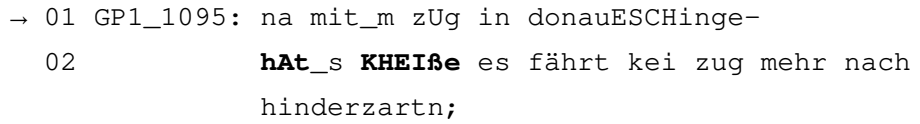

Hier berichtet der Sprecher GP1_1095, wie er 1945 von Tiengen nach Weisweil mit dem Zug fahren wollte. Als er in Donaueschingen ankam, habe er erfahren, dass kein Zug mehr fahre (Z. 02). Die Konstruktion „hAt_s KHEIße“ leitet dabei eine direkte Rede ein. Aktionsartlich handelt es sich hier also bei dem Verb heißen nicht um einen Zustand, sondern um ein Semelfactive. Die Situation ist zwar atelisch, aber auch nicht-durativ. Da zudem andere Indizien fehlen, die für eine unabgeschlossene Sprecherperspektive sprächen, ist diese Beschreibung als abgeschlossen zu betrachten.

\section{Statistische Auswertung}

Für den möglichen Zusammenhang zwischen abgeschlossener/unabgeschlossener Perspektive und Perfekt/Präteritum wurde die Stichprobe von 2140 Belegen ausgewertet. Die Präteritum- und Perfektformen sind mit 1039 bzw. 1101 in etwa gleich verteilt. Die nachfolgende Tabelle 24 zeigt die relative und absolute Verteilung der abgeschlossenen und unabgeschlossenen Perspektive für Perfekt und Präteritum.

23 Denkbar wäre beispielsweise der Satz: „Jedesmal, wenn ich einen Gegenstand gefunden habe, habe ich einen anderen wieder verloren.“, der durch das Adverb jedesmal eine unabgeschlossene Situation anzeigt. 
Tab. 24: Absolute und relative Häufigkeiten der Sprecherperspektive für Perfekt und Präteritum

\begin{tabular}{lccc}
\hline & Unabgeschlossen & Abgeschlossen & Gesamt \\
\hline Perfekt & $648(62,4 \%)$ & $391(37,6 \%)$ & $1039(100 \%)$ \\
Präteritum & $885(80,4 \%)$ & $216(19,6 \%)$ & $1101(100 \%)$ \\
Gesamt & $1533(71,6 \%)$ & $607(28,4 \%)$ & $2140(100 \%)$ \\
\hline
\end{tabular}

Perfekt und Präteritum kommen in unabgeschlossenen Situationen mit 1533 (71,6\%) Belegen häufiger vor als in abgeschlossenen Situationen mit 607 (28,4\%) Belegen. Dieses Ergebnis ist auf den ersten Blick überraschend. Grundsätzlich wäre zu erwarten, dass abgeschlossene Situationen häufiger vorkommen, da die unabgeschlossene Perspektivierung der Rahmensetzung dient und die abgeschlossene die eigentlich für die Erzählung relevante Situation beschreibt (vgl. dazu auch Kap. 4.2.3).

Die auffallend hohe Zahl an unabgeschlossenen Situationen lässt sich durch die Verben erklären, aus denen sich die Stichprobe zusammensetzt. Wie oben beschrieben wurde, hat die Aktionsart einen starken Einfluss auf den Ausdruck von (Un-)Abgeschlossenheit. Für die Auswahl der Stichprobe wurden in diesem Kapitel nur diejenigen Verben herangezogen, die sowohl im Perfekt als auch im Präteritum belegt sind, da die beiden Tempora auch nur bei diesen Verben miteinander konkurrieren können. Die meisten dieser Verben sind aber, wie in Kapitel 3.1.6 deutlich wurde, Zustandsverben (z. B. haben, sein und die Modalverben). So lässt sich die hohe Zahl an Belegen mit unabgeschlossener Situation in der Gesamtmenge sowie beim jeweiligen Tempus erklären. Bei beiden Tempora machen dementsprechend unabgeschlossene Situationen mit 62,4\% (Perfekt) und 80,4\% (Präteritum) den Großteil der Belege aus. Ein Chi-Quadrat-Test, der die Äußerungen mit Perfekt beziehungsweise Präteritum mit unabgeschlossenen und abgeschlossenen Situationen vergleicht, ergibt einen Wert von 85,368 ${ }^{\star \star \star}$; Cramers Phi zeigt mit einem Wert von $\varphi=0,20$ eine schwache Assoziationsstärke an.

Da diese Stichprobe sowohl Zustandsverben enthält, die immer die Aktionsart State ausdrücken, als auch Verben wie kommen und finden, die je nach Verbbedeutung verschiedene Aktionsarten haben, bleibt der genaue Zusammenhang von Aktionsart und (Un-)Abgeschlossenheit zunächst unklar. Um den möglichen Einfluss der Aktionsart auf die (Un-)Abgeschlossenheit offenzulegen, vergleiche ich zunächst den Ausdruck von (Un-)Abgeschlossenheit beim Zustandsverb sein, bei dem jeder Beleg der Aktionsart State zuzurechnen ist. Ich habe hierzu eine weitere Stichprobe aus je 400 Belegen vom Perfekt und Präteritum des Zustandsverbs sein erstellt. Das folgende Beispiel (67) zeigt zwei Äußerungen mit dem 
Verb sein: In der einen wird eine unabgeschlossene Situation dargestellt (Z. 01), in der anderen eine abgeschlossene (Z. 05).

(67) Achern (REDI+FLARS (dt.))

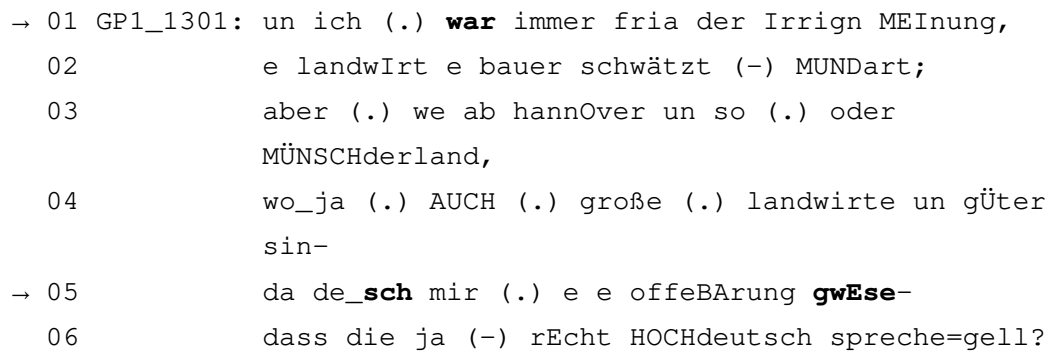

Der Informant GP1_1301 erzählt hier, er wäre früher der Meinung gewesen, Landwirte sprächen grundsätzlich Dialekt (Z. 01). Später habe er jedoch erfahren, dass Landwirte „ab hannOver un so (.) oder MÜNSCHderland“ (Z. 03) sehr hochdeutsch sprächen (Z. 06). Bei der Äußerung in Zeile 01 handelt es sich um eine unabgeschlossene Situation, da auf eine nicht näher definierte Zeitdauer referiert wird. Das wird an den Adverbien „immer“ und „fria“ (Z. 01) deutlich. Die Äußerung in Zeile 05 drückt dagegen eine abgeschlossene Situation aus, da ein singulärer (oder zumindest klar eingegrenzter) Moment der Erkenntnis beschrieben wird.

Tabelle 25 zeigt die relative und absolute Verteilung unabgeschlossener und abgeschlossener Situationen für Perfekt und Präteritum beim Kopulaverb sein.

Tab. 25: Absolute und relative Häufigkeiten von (Un-)Abgeschlossenheit für Perfekt und Präteritum des Verbs sein

\begin{tabular}{lcrc}
\hline & Unabgeschlossen & Abgeschlossen & Gesamt \\
\hline Perfekt & $329(82,3 \%)$ & $71(17,8 \%)$ & $400(100 \%)$ \\
Präteritum & $347(86,8 \%)$ & $53(13,3 \%)$ & $400(100 \%)$ \\
Gesamt & $676(84,5 \%)$ & $124(15,5 \%)$ & $800(100 \%)$ \\
\hline
\end{tabular}

Im Vergleich zur obigen Stichprobe ist hier ein mit 84,5\% insgesamt noch höherer Anteil an unabgeschlossenen Situationen zu erkennen. Während in der obigen Stichprobe beim Ausdruck unabgeschlossener Situationen noch ein relativer Unterschied von $18 \%$ zwischen den beiden Tempora zu sehen war, ist dieser hier mit $82,3 \%$ vs. $86,8 \%$ sehr gering. Ein Chi-Quadrat-Test, der die Äußerungen mit 
Perfekt beziehungsweise Präteritum mit unabgeschlossenen und abgeschlossenen Situationen beim Kopulaverb sein vergleicht, ergibt dementsprechend ein nicht-signifikantes Ergebnis. Das heißt, bei einem Verb wie sein, das im Perfekt und im Präteritum dieselbe Aktionsart hat, gibt es keine Korrelation zwischen den Tempora und (un-)abgeschlossenen Situationen.

In Kapitel 3.1.6 konnte ich aufzeigen, dass der Großteil der Präteritum-Belege der Verben finden, geben, gehen, heißen und kommen der Aktionsart State zuzurechnen ist. Ich vergleiche daher nun den Ausdruck der (Un-)Abgeschlossenheit bei Äußerungen mit diesen Verben. In den Tabellen 26 und 27 ist die absolute und relative Verteilung unabgeschlossener und abgeschlossener Situationen für Perfekt und Präteritum bei den genannten Verben zu sehen.

Tab. 26: Absolute und relative Häufigkeiten von (Un-)Abgeschlossenheit für Perfekt und Präteritum der Verben finden, geben, gehen, heißen und kommen innerhalb des Tempus

\begin{tabular}{lrrr}
\hline & Unabgeschlossen & Abgeschlossen & \multicolumn{1}{c}{ Gesamt } \\
\hline Perfekt & $529(37,3 \%)$ & $889(62,7 \%)$ & $1418(100 \%)$ \\
Präteritum & $538(76,7 \%)$ & $163(23,3 \%)$ & $701(100 \%)$ \\
Gesamt & $1067(50,4 \%)$ & $1052(49,6 \%)$ & $2119(100 \%)$ \\
\hline
\end{tabular}

Auffällig ist hierbei, dass unabgeschlossene und abgeschlossene Situationen mit 50,4\% bzw. 49,6\% fast gleich verteilt sind. Beim Perfekt machen abgeschlossene Situationen mit 62,7\% zu 37,3\% die große Mehrheit der Belege aus; beim Präteritum sind dagegen unabgeschlossene Situationen mit 76,7\% deutlich häufiger als abgeschlossene mit 23,3\%.

Tab. 27: Absolute und relative Häufigkeiten von (Un-)Abgeschlossenheit für Perfekt und Präteritum der Verben finden, geben, gehen, heißen und kommen innerhalb der (Un-)Abgeschlossenheit

\begin{tabular}{lrrr}
\hline & Unabgeschlossen & Abgeschlossen & \multicolumn{1}{c}{ Gesamt } \\
\hline Perfekt & $529(49,6 \%)$ & $889(84,5 \%)$ & $1418(66,9 \%)$ \\
Präteritum & $538(50,4 \%)$ & $163(15,5 \%)$ & $701(33,1 \%)$ \\
Gesamt & $1067(100 \%)$ & $1052(100 \%)$ & $2119(100 \%)$ \\
\hline
\end{tabular}

Vergleicht man, mit welchem Tempus unabgeschlossene und abgeschlossene Situationen korrelieren, wird ersichtlich, dass unabgeschlossene Situationen mit 
beiden Tempora fast gleich häufig ausgedrückt werden (49,6\% vs. 50,4\%); abgeschlossene Situationen dagegen mit 84,5\% zu 15,5\% größtenteils mit Perfekt. Ein Chi-Quadrat-Test, der die Äußerungen mit Perfekt beziehungsweise Präteritum mit unabgeschlossenen und abgeschlossenen Situationen vergleicht, ergibt ei-

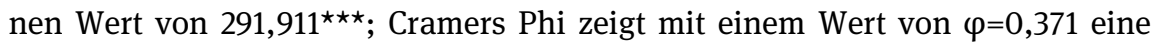
schwache bis mittlere Assoziationsstärke an.

Aufgrund der schwachen Assoziationsstärke in der Zufallsstichprobe zwischen den Tempora und (un-)abgeschlossenen Situationen lässt sich festhalten, dass es im Alemannischen Deutschlands keine aspektuelle Opposition gibt, die durch Perfekt und Präteritum ausgedrückt wird. Das nicht-signifikante Ergebnis für den Vergleich beim Verb sein und die mittlere Assoziationsstärke bei den Verben mit unterschiedlichen Aktionsarten zeigt dagegen, dass die Aktionsart der Belege einen starken Einfluss auf die (Un-)Abgeschlossenheit einer Situation hat. Es erscheint daher sinnvoll, im folgenden Kapitel die Präteritum- und PerfektBelege derjenigen Verben hinsichtlich der Aktionsart zu vergleichen, die je nach Teilbedeutung verschiedene Aktionsarten haben können. Diese sind finden, geben, gehen, heißen und kommen. In Kapitel 3.1.6 habe ich bereits gezeigt, dass die Präteritalformen dieser Verben meistens die Aktionsart State haben. Durch die Betrachtung aller Belege dieser Verben in einem Tempus der einfachen Vergangenheit möchte ich nun untersuchen, ob die Teilbedeutungen mit Aktionsart State vorwiegend im Präteritum ausgedrückt werden.

\subsubsection{Einfluss der Aktionsart auf die Tempuswahl}

Wie eben verdeutlicht wurde, korrelieren Aktionsart und (Un-)Abgeschlossenheit einer Situation miteinander. Dies liegt daran, dass bei Äußerungen, die keine weiteren Indizien für die Zuordnung zu einer abgeschlossenen oder unabgeschlossenen Situation aufweisen, anhand der Aktionsart ersichtlich wird, ob Abgeschlossenheit oder Unabgeschlossenheit ausgedrückt werden. Aktionsarten, die durativ und atelisch sind (Activity und State), weisen in sonst merkmallosen Äußerungen auf unabgeschlossene Situationen hin; die anderen Aktionsarten auf abgeschlossene Situationen. ${ }^{24}$

In Kapitel 3.1.6 konnte ich aufzeigen, dass 16 der 22 präteritumaffinen Verben Zustandsverben sind, d. h. der Aktionsart State zuzuordnen sind. Die Verben finden, geben, gehen, heißen und kommen sind dagegen keine Zustandsverben,

24 Teile dieses Unterkapitels wurden mit einer Teilmenge der Daten bereits in Leonhard (2021) publiziert. 
besitzen aber verschiedene Verbbedeutungen, bei denen mindestens eine ein State ist. Bei diesen Verben stellt sich die Frage, ob es Korrelationen zwischen der Aktionsart State und einem der beiden Tempora gibt. In Kapitel 3.1.6 wurde deutlich, dass deren Präteritalformen hauptsächlich der Aktionsart State zuzuordnen sind. Ich möchte darauf aufbauend nun die Präterital- mit den Perfektformen dieser Verben vergleichen und versuchen, Tendenzen einzelner Aktionsarten zu einem der beiden Tempora aufzudecken. Hierfür vergleiche ich alle Präteritumund Perfekt-Belege der Verben finden, geben, gehen, heißen und kommen, die keinen Gegenwartsbezug haben (vgl. Kap. 3.2.2).

Die nachfolgende Tabelle 28 zeigt die absoluten und relativen Häufigkeiten dieser Verben, die hinsichtlich der Aktionsart untersucht werden sollen.

Tab. 28: Absolute und relative Häufigkeiten von Perfekt und Präteritum der Verben finden, geben, gehen, heißen und kommen im Korpus REDI+FLARS (dt.)

\begin{tabular}{lrrr}
\hline & \multicolumn{1}{c}{ Präteritum } & \multicolumn{1}{c}{ Perfekt } & \multicolumn{1}{c}{ Gesamt } \\
\hline finden & $46(53,5 \%)$ & $40(46,5 \%)$ & $86(100 \%)$ \\
geben & $227(58,1 \%)$ & $164(41,9 \%)$ & $391(100 \%)$ \\
gehen & $144(28,2 \%)$ & $366(71,8 \%)$ & $510(100 \%)$ \\
heißen & $47(29,0 \%)$ & $115(71,0 \%)$ & $162(100 \%)$ \\
kommen & $237(25,3 \%)$ & $698(74,7 \%)$ & $935(100 \%)$ \\
Gesamt & $701(33,6 \%)$ & $1383(66,4 \%)$ & $2084(100 \%)$ \\
\hline
\end{tabular}

Das Perfekt macht $66,4 \%$ der Belege aus. Zwischen den einzelnen Verben gibt es zum Teil große Unterschiede in der Perfekt-Präteritum-Verteilung. Während bei den Verben finden und geben das Präteritum mit 53,5\% bzw. 58,1\% den etwas größeren Anteil aufweist, sind bei den Verben gehen, heißen und kommen nur 28,2\%, 29,0\% bzw. 25,3\% der Belege im Präteritum.

Nachfolgend präsentiere ich die Verteilung der Tempusverwendung nach der Aktionsart für die jeweiligen Verben und anschließend für alle Verben.

Tabelle 29 zeigt die Verteilung der Aktionsarten auf Perfekt und Präteritum beim Verb finden. 
Tab. 29: Absolute und relative Häufigkeiten der Aktionsarten für Perfekt und Präteritum des Verbs finden im Korpus REDI+FLARS (dt.)

\begin{tabular}{lccc}
\hline & Präteritum & Perfekt & Gesamt \\
\hline Achievement & $0(0 \%)$ & $31(100 \%)$ & $31(100 \%)$ \\
State & $46(83,6 \%)$ & $9(16,4 \%)$ & $55(100 \%)$ \\
Gesamt & $46(53,5 \%)$ & $40(46,5 \%)$ & $86(100 \%)$ \\
\hline
\end{tabular}

Wie schon in Kapitel 3.1.6 beschrieben sind alle Präteritumformen des Verbs finden States. Beim Perfekt dagegen sind nur 22,5\% (9 der 40 Belege) States. Die Aktionsart State wird in 83,6\% der Fälle durch Präteritum ausgedrückt. Wir sehen also beim Verb finden für die Verwendung von Perfekt und Präteritum eine Spezialisierung hinsichtlich der Aktionsart.

Beim Verb geben zeigt sich ein ähnliches Bild.

Tab. 30: Absolute und relative Häufigkeiten der Aktionsarten für Perfekt und Präteritum des Verbs geben im Korpus REDI+FLARS (dt.)

\begin{tabular}{lcrr}
\hline & Präteritum & \multicolumn{1}{c}{ Perfekt } & \multicolumn{1}{c}{ Gesamt } \\
\hline Activity & $0(0 \%)$ & $14(100 \%)$ & $14(100 \%)$ \\
Accomplishment & $0(0 \%)$ & $2(100 \%)$ & $2(100 \%)$ \\
Achievement & $1(1,9 \%)$ & $51(98,1 \%)$ & $52(100 \%)$ \\
State & $226(70,0 \%)$ & $97(30,0 \%)$ & $323(100 \%)$ \\
Gesamt & $227(58,1 \%)$ & $164(41,9 \%)$ & $391(100 \%)$ \\
\hline
\end{tabular}

Die Präteritumformen von geben sind zu 99,6\% States. Der einzige PräteritumBeleg, der der Aktionsart Achievement zuzuordnen ist, wurde in Beispiel (31) des Kapitels 3.1.6 vorgestellt. Beim Perfekt sind mit 97 von 164 Belegen (59,1\%) ebenfalls die Mehrzahl der Fälle States. Wie auch bei finden ist beim Verb geben eine Spezialisierung zu beobachten, bei der States größtenteils (d. h. in 70,0\% der Fälle) im Präteritum gebraucht werden.

Tabelle 31 zeigt die Verteilung für das Verb gehen. 
Tab. 31: Relative und absolute Häufigkeiten der Aktionsarten für Perfekt und Präteritum des Verbs gehen im Korpus REDI+FLARS (dt.)

\begin{tabular}{lrrr}
\hline & \multicolumn{1}{c}{ Präteritum } & \multicolumn{1}{c}{ Perfekt } & \multicolumn{1}{c}{ Gesamt } \\
\hline Accomplishment & $27(8,5 \%)$ & $290(91,5 \%)$ & $317(100 \%)$ \\
Achievement & $10(62,5 \%)$ & $6(37,5 \%)$ & $16(100 \%)$ \\
State & $107(60,5 \%)$ & $70(39,5 \%)$ & $177(100 \%)$ \\
Gesamt & $144(28,2 \%)$ & $366(71,8 \%)$ & $510(100 \%)$ \\
\hline
\end{tabular}

Auch hier sind die Präteritum-Belege mit 107 von 144 (74,3\%) mehrheitlich States. Die Perfekt-Belege sind dagegen nur in 70 von 366 Fällen $(19,1 \%)$ States. Die überwiegende Mehrzahl der Perfekt-Belege ist mit 79,2\% der Aktionsart Accomplishment zuzurechnen. Auffallend ist bei diesem Verb, dass die Aktionsart Achievement häufiger mit Präteritum als mit Perfekt gebildet wird (62,5\% vs. $37,5 \%)$. Dabei handelt es sich wohl um eine lexikalische Besonderheit, da alle Achievements auf das Verb losgehen zurückzuführen sind. Die Aktionsart State wird mit $107 \mathrm{zu} 70$ (60,5\%) in der Mehrzahl der Fälle im Präteritum gebraucht. Der Unterschied zwischen den beiden Tempora ist allerdings weniger deutlich als bei den eben vorgestellten Verben finden und geben.

Die Verteilung für das Verb heißen ist in Tabelle 32 abgebildet.

Tab. 32: Absolute und relative Häufigkeiten der Aktionsarten für Perfekt und Präteritum des Verbs heißen im Korpus REDI+FLARS (dt.)

\begin{tabular}{lrrr}
\hline & Präteritum & \multicolumn{1}{c}{ Perfekt } & \multicolumn{1}{c}{ Gesamt } \\
\hline Semelfactive & $9(12,0 \%)$ & $66(88,0 \%)$ & $75(100 \%)$ \\
State & $38(43,7 \%)$ & $49(56,3 \%)$ & $87(100 \%)$ \\
Gesamt & $47(29,0 \%)$ & $115(71,0 \%)$ & $162(100 \%)$ \\
\hline
\end{tabular}

Die Präteritumformen sind in 38 von 47 Fällen (80,9\%) States und in neun von 47 Fällen (19,1\%) Semelfactives. Beim Perfekt sind dagegen nur 49 von 115 Belegen $(42,6 \%)$ States; die restlichen 66 Belege (57,4\%) sind Semelfactives. Im Gegensatz zu finden, geben und gehen werden alle Aktionsarten häufiger mit Perfekt als mit Präteritum gebildet. Bei der Aktionsart Semelfactive macht das Perfekt 88,0\% und bei State $56,3 \%$ aus.

Tabelle 33 zeigt die Verteilung für das Verb kommen. 
Tab. 33: Absolute und relative Häufigkeiten der Aktionsarten für Perfekt und Präteritum des Verbs kommen im Korpus REDI+FLARS (dt.)

\begin{tabular}{lrrl}
\hline & \multicolumn{1}{c}{ Präteritum } & \multicolumn{1}{c}{ Perfekt } & Gesamt \\
\hline Accomplishment & $109(15,8 \%)$ & $583(84,2 \%)$ & $692(100 \%)$ \\
Achievement & $17(16,7 \%)$ & $85(83,3 \%)$ & $102(100 \%)$ \\
State & $111(78,7 \%)$ & $30(21,3 \%)$ & $141(100 \%)$ \\
Gesamt & $237(25,3 \%)$ & $698(74,7 \%)$ & $935(100 \%)$ \\
\hline
\end{tabular}

Bei den Präteritalformen des Verbs kommen machen States mit 111 von 237 Belegen 46,8\% aus. Fast ebenso viele Präteritalformen sind der Aktionsart Accomplishment zuzuordnen (46,0\%). Bei den Perfektformen sind demgegenüber nur 30 von 698 Belegen (4,3\%) States. Accomplishments machen mit 583 von 698 Belegen (83,5\%) die große Mehrzahl aller Perfekte aus. Während Accomplishments und Achievements mit 84,2\% bzw. 83,3\% häufiger im Perfekt als im Präteritum gebraucht werden, werden die meisten States (78,7\%) mit Präteritum gebildet.

Die nachfolgende Tabelle 34 stellt die Verteilung der Tempusverwendung nach der Aktionsart aller Verben dar.

Tab. 34: Absolute und relative Häufigkeiten der Aktionsarten aller Verben für Präteritum und Perfekt in REDI+FLARS (dt.)

\begin{tabular}{lcrlr}
\hline & Präteritum & \multicolumn{1}{c}{ Perfekt } & \multicolumn{1}{c}{ Gesamt } \\
\hline Activity & $0(0 \%)$ & $14(100 \%)$ & $14(100 \%)$ \\
Accomplishment & $136(13,5 \%)$ & $875(86,5 \%)$ & $1011(100 \%)$ \\
Achievement & $28(13,9 \%)$ & $173(86,1 \%)$ & $201(100 \%)$ \\
Semelfactive & $9(12,0 \%)$ & $66(88,0 \%)$ & $75(100 \%)$ \\
State & $528(67,4 \%)$ & $255(32,6 \%)$ & $783(100 \%)$ \\
Gesamt & $701(33,6 \%)$ & $1383(66,4 \%)$ & $2084(100 \%)$ \\
\hline
\end{tabular}

Mit Ausnahme der States werden alle Aktionsarten mit relativen Häufigkeiten zwischen $86,1 \%$ und $100 \%$ überwiegend im Perfekt gebraucht. States werden dagegen in $67,4 \%$ mit Präteritum gebildet. Um zu überprüfen, ob diese Verteilungen signifikant sind, habe ich eine binär logistische Regressionsanalyse für den Vergleich der Aktionsart State mit den restlichen Aktionsarten bei den beiden Tempora durchgeführt. Mit der Regressionsanalyse kann der Einfluss unabhängiger Variablen auf eine abhängige Variable gemessen werden. Die unabhängige 
Variable ist hier Aktionsart (d. h. State oder eine andere Aktionsart); die abhängige Variable ist das gewählte Tempus (d. h. Perfekt oder Präteritum). Diese Regressionsanalyse ergibt einen höchstsignifikanten Wert für den Einfluss der Aktionsart State auf die Wahl des Tempus. Wenn ein Verb anstelle der Aktionsart State die Aktionsart Accomplishment hat, ist die Wahrscheinlichkeit für Perfekt anstelle von Präteritum das 13,3-fache; bei der Aktionsart Achievement das 12,8fache und bei der Aktionsart Semelfactive das 15,2-fache. Für die Aktionsart Activity ergibt die binär logistische Regressionsanalyse kein signifikantes Ergebnis. Die Aktionsart des Verbs hat also einen maßgeblichen Einfluss auf die Wahl des Tempus.

Das Vorhandensein der beiden Tempora der einfachen Vergangenheit führt also zu einer Spezialisierung, bei der States mehrheitlich durch Präteritum ausgedrückt werden. Bei einigen Verben ist diese Spezialisierung stärker ausgeprägt als bei anderen. Während bei den Verben finden und kommen 83,6\% bzw. 78,7\% der States im Präteritum gebraucht werden, sind es bei heißen nur 43,7\%. Diese Verteilung deckt sich mit den Ergebnissen aus Kapitel 3.1.6. In diesem Kapitel konnte ich zeigen, dass alle Präteritalformen auf Zustandsverben zurückgehen oder auf stative Teilbedeutungen von Verben, die nicht per se Zustandsverben sind. Damit lässt sich auch die Vermutung bestätigen, dass das erhöhte Vorkommen unabgeschlossener Situationen auf die Aktionsart des Verbs zurückzuführen ist.

In den folgenden beiden Kapiteln gehe ich auf die morpho-syntaktischen Unterschiede der beiden Tempora ein und versuche aufzuzeigen, welche Folgen sich aus der unterschiedlichen Anzahl an Verbteilen für die Verbstellung (Kap. 3.2.5) und die Passivbildung (Kap. 3.2.6) ergeben.

\subsubsection{Einfluss der Verbstellung auf die Tempuswahl}

In diesem Kapitel soll untersucht werden, ob die Position des finiten Verbs einen Einfluss auf die Wahl zwischen Perfekt und Präteritum hat. Um mögliche semantische Unterschiede auszuschließen, habe ich für diese Untersuchung ein Korpus aus den Verben dürfen, haben, können, müssen, sein, sollen, stehen, wissen und wollen zusammengestellt. Es setzt sich aus jeweils 650 Belegen für Perfekt und Präteritum zusammen, die keinen Gegenwartsbezug haben. Für die Untersuchung eines möglichen Einflusses der Verbstellung auf die Tempuswahl habe ich die Belege nach der Stellung des finiten Verbs in der Äußerung kodiert. Die nachfolgenden Beispiele zeigen Äußerungen im Perfekt beziehungsweise Präteritum in Verberst- (68), Verbzweit- (69) und Verbletztstellung (70). Deklarativsätze mit 
nicht-realisiertem Subjekt, bei denen das Verb an erster Position steht, wurden zu den Verbzweitsätzen gezählt, da bei diesen keine Unterschiede zu Verbzweitsätzen mit realisiertem Subjekt anzunehmen sind. Alle Verberstsätze im Korpus REDI+FLARS (dt.) sind Fragesätze. Uneingeleitete Konditional- oder Konzessivsätze sind im Korpus nicht enthalten.

(68) Waldkirch (REDI+FLARS (dt.))

$\rightarrow 01$ GP1_1086: wAre sie scho_mal im KAIserstuhl?

02 EX1_1051: jA wir warn schon_mal in BREIsach;

(69) Weil (REDI+FLARS (dt.))

$\rightarrow 01$ GP1_1248: die hen AU e tolle diaLEKT kha da.

(70) Böttingen (REDI+FLARS (dt.))

$\rightarrow 01$ GP6_657: WAs_i grad SAge wollt;

Die nachfolgende Tabelle 35 zeigt die absolute und relative Verteilung der Perfekt- und Präteritum-Belege nach der Position des finiten Verbs.

Tab. 35: Absolute und relative Häufigkeiten von Perfekt und Präteritum nach der Position des finiten Verbs in REDI+FLARS (dt.) innerhalb des Tempus

\begin{tabular}{lcrcr}
\hline & V1-Stellung & V2-Stellung & VL-Stellung & \multicolumn{1}{c}{ Gesamt } \\
\hline Perfekt & $2(0,31 \%)$ & $595(91,5 \%)$ & $53(8,2 \%)$ & $650(100 \%)$ \\
Präteritum & $1(0,15 \%)$ & $562(86,5 \%)$ & $87(13,4 \%)$ & $650(100 \%)$ \\
Gesamt & $3(0,23 \%)$ & $1157(89,0 \%)$ & $140(10,8 \%)$ & $1300(100 \%)$ \\
\hline
\end{tabular}

Die Verbzweitstellungen machen bei Perfekt und Präteritum mit 91,5\% bzw. 86,5\% jeweils den Großteil der Verwendungen aus. Angesichts der grundsätzlichen Tendenz gesprochener Sprache zur Parataxe (vgl. Rödel 2007: 118) verwundert dieses Ergebnis nicht.

Bei beiden Tempora entfällt auf Verberststellungen erwartungsgemäß der kleinste Teil aller Verwendungen. Beim Perfekt machen Verberstpositionen 0,31\% aller Belege aus; beim Präteritum nur 0,15\%.

Eine binär logistische Regressionsanalyse ergibt für die Wahl des Tempus bei Verberststellungen einen statistisch nicht-signifikanten Wert. Dagegen weicht die Anzahl der Verbletztstellungen hochsignifikant $(p=0,003)$ voneinander $a b$. 
Während beim Präteritum 13,4\% der Belege in Verbletztposition auftreten, sind es beim Perfekt nur 8,2\%. Dabei beträgt die Wahrscheinlichkeit für Präteritum bei VL-Sätzen das 1,7-fache. Ein Chi-Quadrat-Test ergibt einen hochsignifikanten Wert von $\mathrm{p}=0,003\left(\mathrm{X}^{2}(2, \mathrm{n}=1300)=9,621\right)$. Es zeigt sich also eine leichte Tendenz zur vermehrten Präteritumverwendung. Gemessen an dem niedrigen Chi-Quadrat-Wert und dem niedrigen Chancenverhältnis von 0,58 kann die Verbstellung als marginaler Faktor betrachtet werden.

\subsubsection{Einfluss der Diathese auf die Tempuswahl}

In Kapitel 3.2.1 habe ich die These vorgestellt, die Klammerbildung sei ein entscheidender Vorteil des Perfekts gegenüber dem Präteritum (vgl. Leiss 1992: 278 und Fischer 2018: 129). Denkbar wäre daher, dass in Kontexten, in denen die rechte Satzklammer unabhängig vom Tempus besetzt ist, eine Tendenz zur Präteritumverwendung festzustellen ist. Nach den eben vorgestellten Verbletztsätzen möchte ich nun der Frage nachgehen, ob in Passivkonstruktionen ein Unterschied in der Tempuswahl zu beobachten ist.

Ich betrachte hierzu alle Vorgangspassive, die mit werden gebildet werden. Für das Zustandspassiv habe ich 400 Präteritum- und 400 Perfekt-Belege des Verbs sein durchsucht. Lediglich acht Belege (sechs Präterital- und zwei PerfektBelege) waren Hilfsverben zur Passivbildung. Die restlichen 792 Belege waren Kopulaverben, da Hilfsverben zur Plusquamperfektbildung nicht berücksichtigt wurden (vgl. Kap. 3). Im Vergleich zum Kopulaverb sein kommen Hilfsverben zur Bildung des Zustandspassivs also fast nicht vor.

Die folgenden Beispiele illustrieren die Verwendung des Verbs werden als Kopulaverb (71) und als Hilfsverb zur Bildung des Vorgangspassivs (72).

(71) Kappel-Grafenhausen (REDI+FLARS (dt.))

$\rightarrow 01$ GP1_1364: mi: (.) mi: schwEschder isch dann SCHWANger worre;

(72) Au am Rhein (REDI+FLARS (dt.))

$\rightarrow 01$ GP1_1318: bei uns wurd nie (.) HOCHdeutsch gschwätzt daheim, 02 (.) ei also wIrklich NIE- 
Die Tabellen 36 und 37 zeigen die Verteilung der Präteritalformen des Verbs werden auf Voll- und auf Hilfsverben zur Bildung des Vorgangspassivs bei Perfekt und Präteritum.

Tab. 36: Absolute und relative Häufigkeiten von Perfekt und Präteritum bei werden als Vollund als Hilfsverb in REDI+FLARS (dt.)

\begin{tabular}{lrrr}
\hline & Kopulaverb & Hilfsverb & Gesamt \\
\hline Perfekt & $169(98,8 \%)$ & $2(1,2 \%)$ & $171(100 \%)$ \\
Präteritum & $14(8,3 \%)$ & $155(91,7 \%)$ & $169(100 \%)$ \\
Gesamt & $183(53,8 \%)$ & $157(46,2 \%)$ & $340(100 \%)$ \\
\hline
\end{tabular}

169 der 171 Perfektformen von werden sind Kopulaverben (98,8\%). Demgegenüber sind mit 155 von 169 Belegen (91,7\%) fast alle Präteritalformen von werden Hilfsverben.

Tab. 37: Absolute und relative Häufigkeiten von werden als Voll- und als Hilfsverb bei Perfekt und Präteritum in REDI+FLARS (dt.)

\begin{tabular}{lrrr}
\hline & \multicolumn{1}{c}{ Kopulaverb } & \multicolumn{1}{c}{ Hilfsverb } & \multicolumn{1}{c}{ Gesamt } \\
\hline Perfekt & $169(92,3 \%)$ & $2(1,3 \%)$ & $171(50,3 \%)$ \\
Präteritum & $14(7,7 \%)$ & $155(98,7 \%)$ & $169(49,7 \%)$ \\
Gesamt & $183(100 \%)$ & $157(100 \%)$ & $340(100 \%)$ \\
\hline
\end{tabular}

Das Kopulaverb werden wird mit 169 von 183 Belegen (92,3\%) fast immer im Perfekt gebraucht. Das Hilfsverb werden ist dagegen mit 155 von 157 Belegen (98,7\%) beinahe ausschließlich im Präteritum.

Ein Chi-Quadrat-Test, der das Verb werden als Voll- und als Hilfsverb mit dem Gebrauch im Perfekt und Präteritum vergleicht, ergibt einen Wert von $280,384^{\star \star \star}$; Cramers Phi zeigt mit einem Wert von $\varphi=0,908$ eine extrem starke Assoziation an. Für das Vorgangspassiv lässt sich also eine fast perfekte Spezialisierung erkennen, wobei die Präteritumformen zur Realisierung des Hilfsverbs und die Perfektformen zur Realisierung des Kopulaverbs gebraucht werden. Es erscheint dabei unwahrscheinlich, dass diese Verteilung alleine auf die Besetzung der rechten Satzklammer bei Passivkonstruktionen zurückgeführt werden kann. Dagegen spricht vor allen Dingen das Ergebnis aus Kapitel 3.2.5, das für Verbletztpositionen des finiten Verbs nur eine schwache Assoziation ergab. 
Stattdessen scheint die Passivbildung an sich Einfluss auf die Wahl des Tempus zu haben. Dabei ist nicht verwunderlich, dass fast alle Präteritum-Belege Hilfsverben sind. Schließlich ist das Kopulaverb werden kein Zustandsverb und besitzt auch keine stative Teilbedeutung, was auf alle anderen präteritumaffinen Verben zutrifft. Eine mögliche Erklärung für das Ergebnis könnte in der Semantik der Passivkonstruktionen liegen: Passivbildungen ähneln semantisch den Konstruktionen mit Zustandsverb dadurch, dass das Subjekt kein Agens ist und der beschriebene Vorgang nicht-dynamisch ist, d. h. keinen Energieaufwand des Partizipanten beinhaltet.

Inwiefern diese Überlegungen auch auf das Zustandspassiv mit sein zutreffen, kann ausgehend von meiner Stichprobe von jeweils 400 Belegen für Präteritum und Perfekt aufgrund einer zu geringen Anzahl an Hilfsverben nicht festgestellt werden.

\subsubsection{Zusammenfassung}

Ich bin in diesem Kapitel der Frage nachgegangen, ob sich die beiden Tempora Perfekt und Präteritum im Korpus REDI+FLARS (dt.) semantisch oder in ihren Verwendungskontexten unterscheiden. Dazu habe ich diejenigen Verben miteinander verglichen, die in beiden Tempora vorkommen.

Zunächst untersuchte ich eine mögliche Gegenwartsrelevanz der beiden Tempora anhand einer Stichprobe aus 2187 Belegen (1101 Präterita und 1086 Perfekte). Eine Gegenwartsrelevanz habe ich allen Situationen zugeschrieben, die in der Vergangenheit abgeschlossen sind und sich auf die Sprechzeit beziehen. Es bestätigte sich die Annahme, dass das Perfekt im Gegensatz zum Präteritum Gegenwartsrelevanz haben kann. In meiner Stichprobe hatten allerdings nur 4,3\% der Perfekt-Belege eine solche Gegenwartsrelevanz. Diese geringe Zahl lässt sich mit der Art der Daten erklären. Der Großteil der Perfekt- und Präteritum-Belege geht auf Erzählungen zurück, die keinen Bezug zur Gegenwart haben. Die wenigen Fälle, in denen Perfekte Gegenwartsrelevanz besitzen, kommen in Interaktionen der Gewährspersonen mit den Interviewern oder in Erzählungen vor, die zeitlich nahe zum Sprechzeitpunkt liegen. Auch wenn nur wenige Fälle Gegenwartsrelevanz haben, lässt dieses Ergebnis den Schluss zu, dass das Perfekt im Gegensatz zum Präteritum zwei Lesarten hat: eine ohne Gegenwartsrelevanz und eine mit Gegenwartsrelevanz. Um weitere Faktoren zu ermitteln, die einen Einfluss auf die Wahl des Tempus haben können, habe ich für die folgenden Untersuchungen nur noch die Präteritum- und Perfektformen ohne Gegenwartsbezug verglichen. 
Für den Zusammenhang zwischen Tempus und (Un-)Abgeschlossenheit zeigte sich, dass die Tempora Perfekt und Präteritum nicht zur Markierung der aspektuellen Distinktion perfektiv/imperfektiv dienen. Ein Vergleich der Stichprobe von 2140 Belegen zeigte eine ungewöhnlich hohe Zahl an Belegen mit unabgeschlossener Situation. Dies ist zum einen dadurch zu erklären, dass viele Erzählungen von regelmäßigen oder wiederkehrenden Ereignissen handeln („Früher habe ich immer...“); zum anderen aber auch dadurch, dass nur Verben verglichen wurden, die im Perfekt und im Präteritum belegt sind. Es handelt sich also größtenteils um Zustandsverben oder um Verben, die eine stative Teilbedeutung haben. Die Zufallsstichprobe ergab für den Vergleich Perfekt-Präteritum zwar eine schwache Korrelation, wobei beim Präteritum deutlich mehr Belege den unabgeschlossenen Situationen zuzurechnen sind als beim Perfekt. Allerdings ist diese Korrelation auf die Aktionsart der Verben zurückzuführen. In der Zufallsstichprobe waren neben Verben, die verschiedene Aktionsarten besitzen wie kommen, finden und geben, vor allen Dingen Zustandsverben enthalten, die immer der Aktionsart State zuzuordnen sind.

Um den Faktor Aktionsart auszuschließen, habe ich die beiden Tempora und die (Un-)Abgeschlossenheit einer Situation nur beim Verb sein verglichen. Dieser Vergleich ergab keine signifikante Korrelation, $d$. h. bei identischer Aktionsart ist kein Unterschied zwischen Präteritum und Perfekt hinsichtlich der (Un-)Abgeschlossenheit von Situationen auszumachen. Umgekehrt zeigte sich beim Vergleich derjenigen Verben, die verschiedene Aktionsarten besitzen, eine mittelstarke Korrelation zwischen abgeschlossenen Situationen und Tempus Perfekt einerseits sowie unabgeschlossenen Situationen und Tempus Präteritum andererseits. Der Grund für die Korrelation zwischen der (Un-)Abgeschlossenheit und der Aktionsart ist, dass bei Äußerungen, die keine weiteren Indizien für die Zuordnung zu unabgeschlossenen oder abgeschlossenen Situationen aufweisen, anhand der Aktionsart deutlich wird, welche Perspektive eingenommen wird. Eine besondere Bedeutung hat hier die Aktionsart State, die bei sonst merkmallosen Äußerungen unabgeschlossene Situationen anzeigt.

Um den Zusammenhang zwischen Präteritum und der Aktionsart State aufzudecken, verglich ich anschließend alle Perfekt- und Präteritum-Belege der Verben miteinander, die verschiedene Aktionsarten haben, das sind: finden, geben, gehen, heißen und kommen. Dabei stellte sich heraus, dass States mehrheitlich durch Präteritum ausgedrückt werden. Bei einigen Verben ist diese Spezialisierung stärker ausgeprägt als bei anderen. Während bei den Verben finden und kommen 83,6\% bzw. 78,7\% der States im Präteritum gebraucht werden, sind es bei heißen nur 43,7\%. 
Semantisch lassen sich Perfekt und Präteritum also durch die Aktionsarten der Verben unterscheiden. Daneben stellte sich die Frage, ob die beiden Tempora auch hinsichtlich morpho-syntaktischer Verschiedenheiten in unterschiedlichen Kontexten gebraucht werden.

Als ein großer Vorteil des Perfekts gegenüber dem Präteritum wird die Verbalklammer (mit Besetzung der linken und rechten Satzklammer) betrachtet, die das Perfekt bildet. Leiss (1992: 278) und Fischer (2018: 129) erkennen, dass Verben, die zur Klammerbildung neigen, das Präteritum (häufiger) erhalten als Verben, die keine Klammer bilden. Dies lässt sich dadurch erklären, dass das Perfekt bei Verben, die ohnehin eine Klammer bilden, keinen Vorteil gegenüber dem Präteritum hat. Ausgehend von dieser Überlegung untersuchte ich Kontexte, in denen die rechte Satzklammer unabhängig vom Tempus besetzt ist. Ich verglich daher zum einen Verbzweitsätze mit Verbletztsätzen und zum anderen Passiv- mit Aktivkonstruktionen bei den beiden Tempora. Der Vergleich der Position des finiten Verbs offenbarte eine schwache Korrelation von Verbletztsätzen und Präterita.

Da das Zustandspassiv mit sein zu selten belegt war, konnte hierfür keine Analyse angestellt werden. Beim Vorgangspassiv zeigte sich allerdings, dass dieses fast ausschließlich im Präteritum (in 98,7\% der Fälle) gebraucht wird, während Konstruktionen des Kopulaverbs werden größtenteils mit Perfekt (in 92,3\% der Fälle) gebildet werden. Damit bestätigt sich die Beobachtung Siebergs, der für die Rheinische Umgangssprache nachweist, dass das Perfekt von Passiv- und Modalverbkonstruktionen vermieden und stattdessen das Präteritum gebraucht wird (Sieberg 1984: 95).

Ich vermute daher, dass die Klammerbildung des Perfekts keinen (starken) Einfluss auf die Wahl zwischen Perfekt und Präteritum hat. Stattdessen scheint das Vorgangspassiv semantisch zum Präteritum zu tendieren. Eine Ähnlichkeit zwischen Vorgangspassivbildungen und Konstruktionen mit Zustandsverb besteht, da in beiden Fällen das Subjekt kein Agens ist und der beschriebene Vorgang nicht-dynamisch ist, d. h. keinen Energieaufwand des Partizipanten beinhaltet.

\subsection{Fazit}

Ziel dieses Kapitels war es, die jüngere sprachgeschichtliche Entwicklung und die Semantik des Präteritums im Alemannischen Deutschlands zu beschreiben. Hierzu habe ich zunächst die Entwicklung des Präteritums von 1974 bis 2013 mit Hilfe der beiden Teilkorpora SSA (1974-1985) und REDI+FLARS (dt.) (2007-2013) betrachtet. Es zeigte sich, dass das Präteritum spätestens seit Mitte der 1970er 
Jahre im Alemannischen Deutschlands an Häufigkeit zunimmt. Das Präteritum wird im Untersuchungsgebiet, anders als im Großteil der Forschungsliteratur angenommen, nicht nur in standardnahen Äußerungen verwendet, um vornehm und formell (vgl. Widén 1970: 283) oder besonders standardsprachlich und schriftdeutsch zu klingen (vgl. Fischer 2018: 213). Tatsächlich machte diese Verwendung des Präteritums, die nicht Teil des eigentlichen Dialekts ist, nur einen sehr geringen Teil aus. Der größte Teil der Präteritalverwendungen wird in dialektalen Sprechweisen geäußert und ist daher als Teil des eigentlichen Dialekts zu betrachten. Da ich das Alemannische Deutschlands untersuche, berücksichtige ich lediglich diese Präteritalformen.

Fast alle Präteritalformen weisen regiolektale Merkmale wie Palatalisierung, Tilgung und Lenisierung auf. Im Gegensatz zu den Partizipien existieren aber keine dialektspezifischen Formen. Daraus lässt sich schließen, dass das Präteritum aus dem Standard entlehnt und phonologisch an das Badischalemannische assimiliert wurde. Obwohl das Präteritum durch den Kontakt mit dem Standard in das Badischalemannische wiedereingedrungen ist, zeigte sich beim Vergleich des Dialektalitätsgrads mit der Präteritumfrequenz der Sprecher nur eine schwache bis mittlere Korrelation. Das Präteritum wird von allen Sprechern verwendet und durchdringt damit alle Sprachschichten. Dieser Umstand und die Assimilation an den Regiolekt deuten darauf hin, dass das Präteritum als Teil des Alemannischen Südwestdeutschlands betrachtet werden muss.

Ich konnte beim Vergleich des älteren Korpus SSA mit dem jüngeren Korpus REDI+FLARS (dt.) eine massive Zunahme der arealen Verteilung, der Tokenfrequenz und der Verbtypes, die Präteritum bilden, feststellen. Es war zu beobachten, dass bei fast allen untersuchten Verben die areale Ausbreitung und die Tokenfrequenz stark zugenommen haben. Während im SSA abgesehen von den Präteritalformen von sein und sollen in den meisten Untersuchungsorten keine Präteritumformen zu finden waren, kommen in REDI+FLARS (dt.) in jedem Untersuchungsort auch andere Präteritalformen als die von sein und sollen vor. Der Apparent-Time-Vergleich der älteren mit der jüngeren Sprechergruppe in REDI+FLARS (dt.) offenbarte, dass die Verwendung des Präteritums noch weiter zunimmt. Abgesehen von den Verben sitzen, sollen und stehen nimmt die relative Häufigkeit des Präteritums (im Vergleich zum Perfekt) deutlich zu. Sollen ist in der älteren Sprechergruppe mit 93,3\% bereits so häufig, dass eine weitere starke Zunahme der Präteritumfrequenz nicht mehr möglich ist. Die Verben wissen (4,1mal so häufig), finden (5,0-mal so häufig), kennen (5,4-mal so häufig) und denken (7,0-mal so häufig) werden von jüngeren Sprechern sogar deutlich öfter im Präteritum gebraucht. 
Mit Hilfe eines Vergleichs der Einwohnerzahl der Orte mit der Anzahl an verwendeten Verben konnte gezeigt werden, dass keine signifikante Korrelation zwischen der Größe des Ortes und der Präteritumverwendung besteht. Die Präteritumverwendung beschränkt sich also, anders als von Rowley (1983: 165) vermutet, nicht auf größere Orte, sondern umfasst das gesamte Untersuchungsgebiet.

Auch die relative Frequenz des Präteritums (verglichen mit der des Perfekts) aller Verben, die im Gesamtkorpus mindestens zweimal gebraucht werden, hat vom SSA zu REDI+FLARS (dt.) zugenommen, sodass die relative Frequenz des Präteritums bei diesen Verben in REDI+FLARS (dt.) zwischen 7,9\% und 90,3\% beträgt.

Zudem wurde klar, dass fast nur Zustandsverben ein Präteritum bilden (16 der 22 präteritumaffinen Verben sind Zustandsverben). Fünf der sechs restlichen präteritumaffinen Verben haben eine stative Teilbedeutung und werden (fast) ausschließlich in dieser Teilbedeutung im Präteritum gebraucht. Eine stative Teilbedeutung ist von der Grundbedeutung verschieden und aktionsartlich als State zu beurteilen. Fischer stellt Bezug nehmend auf Harnisch (1997: 120-123) einen stärkeren Präteritumerhalt bei irregulären und starken Verben fest, die eine imperfektive Verbsemantik haben (Fischer 2018: 390). Die wieder in den Dialekt eingedrungenen Präteritalformen betreffen also genau die Verben, die besonders resistent gegenüber dem Präteritumschwund waren. Diese Verben sind daher als besonders präteritumaffin zu bezeichnen. Das Wiedereindringen des Präteritums spiegelt größtenteils die Abbauhierarchie des Präteritumschwunds nach Verbklassen wider, die Fischer (2018) vorstellt. Dabei ist das Verb sein besonders resistent, gefolgt von Modal-/Hilfsverben, irregulären/starken Verben und schwachen Verben.

Die Entstehung von Präteritumformen bei Zustandsverben und Verben mit stativer Teilbedeutung hat im Gegenwartsalemannischen zu einer Spezialisierung geführt, bei der die Aktionsart State größtenteils mit Präteritum ausgedrückt wird und die restlichen Aktionsarten mit Perfekt. Dies wiederum sorgt für einen Zusammenhang zwischen Präteritum und unabgeschlossenen Situationen. Beim stichprobenhaften Vergleich der Perfekt- und Präteritumformen des Verbs sein war allerdings keine Korrelation zwischen einem der beiden Tempora und (un-)abgeschlossenen Situationen zu sehen. Daraus wurde ersichtlich, dass Perfekt und Präteritum nicht selbst die (Un-)Abgeschlossenheit von Situationen markieren. Allerdings unterstützt die Tempusvariation die Distinktion unabgeschlossen/abgeschlossen durch die Festlegung des Präteritums auf Zustände. Da in vielen Fällen nur die Aktionsart einer Äußerung die abgeschlossene oder unabgeschlossene Situation markiert, übernehmen States dort eine tragende Rolle. Zustände deuten (genauso wie Activitys, die aber bei präteritumaffinen Verben 
kaum vorkommen), ohne andere gegenteilige Indizien, auf Unabgeschlossenheit hin. Da die meisten Zustände mit Präteritum ausgedrückt werden, korrelieren Präteritum und Unabgeschlossenheit. Wir stellen also fest, dass das Vorhandensein zweier Tempora der einfachen Vergangenheit indirekt zur Markierung der (Un-)Abgeschlossenheit von Situationen dient, indem die Aktionsart State beim Präteritum häufig unabgeschlossene Situationen vermittelt. Perfekt und Präteritum drücken aber nicht den perfektiven und imperfektiven Aspekt aus.

Auch das Vorgangspassiv hat eine Spezialisierung erfahren. Es zeigte sich, dass Vorgangspassive fast ausschließlich im Präteritum (in 98,7\% der Fälle) und Konstruktionen des Kopulaverbs werden größtenteils im Perfekt (in 92,3\% der Fälle) gebraucht werden. Als ein großer Vorteil des Perfekts gegenüber dem Präteritum wird die Verbalklammer (mit Besetzung der linken und rechten Satzklammer) betrachtet, die das Perfekt bildet. Leiss (1992: 278) und Fischer (2018: 129) stellen fest, dass Verben, die zur Klammerbildung neigen, das Präteritum (häufiger) erhalten als Verben, die keine Klammer bilden. Dies lässt sich dadurch erklären, dass das Perfekt bei Verben, die ohnehin eine Klammer bilden, keinen Vorteil gegenüber dem Präteritum hat. Die Spezialisierung beim Vorgangspassiv ist allerdings nicht auf die Besetzung der rechten Satzklammer bei Passivkonstruktionen zurückzuführen, da nur ein geringer Zusammenhang zwischen Verbzweit-/Verbletztsätzen und Perfekt/Präteritum festzustellen war. Stattdessen scheint das Vorgangspassiv semantisch zum Präteritum zu tendieren. Es gibt eine Ähnlichkeit zwischen Vorgangspassivbildungen und Konstruktionen mit Zustandsverb, da in beiden Fällen das Subjekt kein Agens und der beschriebene Vorgang nicht-dynamisch ist, d. h. keinen Energieaufwand des Partizipanten beinhaltet.

Darüber hinaus bestätigte sich die Annahme, dass das Perfekt im Gegensatz zum Präteritum Gegenwartsrelevanz haben kann. In der Stichprobe, die in dieser Arbeit herangezogen wurde, hatten allerdings nur 4,3\% der Perfekt-Belege Gegenwartsrelevanz. Diese geringe Zahl könnte mit der Art der Daten begründet werden: Der Großteil der Perfekt- und Präteritum-Belege geht auf Erzählungen ohne Bezug zur Gegenwart zurück. Die wenigen Fälle, in denen das Perfekt Gegenwartsrelevanz hat, stammen aus Interaktionen der Gewährspersonen mit den Interviewern oder aus Erzählungen, die zeitlich nahe zum Sprechzeitpunkt liegen. Auch wenn nur wenige Perfekt-Belege Gegenwartsrelevanz besitzen, lässt dieses Ergebnis den Schluss zu, dass das Perfekt im Gegensatz zum Präteritum zwei Lesarten hat: eine ohne und eine mit Gegenwartsrelevanz.

Bei der Betrachtung des Systems der Vergangenheitstempora im Alemannischen Deutschlands stellen wir also fest, dass das Präteritum spätestens seit Mitte der 1970er Jahre durch den Kontakt mit dem Standard in das Alemannische 
wiedereingedrungen ist und an Häufigkeit stark zugenommen hat. Es wurde phonologisch assimiliert und ist nun Teil des eigentlichen Dialekts. Dieses Vorhandensein zweier Tempora der einfachen Vergangenheit hat zu einer Spezialisierung geführt, bei der Vorgangspassive und Verben mit Aktionsart State mit Präteritum ausgedrückt werden. Durch diese Spezialisierung des Präteritums auf States übernimmt dieses Tempus eine aspektähnliche Funktion, die grammatisch sonst - genau wie im Standarddeutschen - nicht ausgedrückt werden kann. 